TRANSACTIONS OF THE

AMERICAN MATHEMATICAL SOCIETY

Volume 348, Number 6, June 1996

\title{
HYPERBOLIC GROUPS AND THEIR QUOTIENTS OF BOUNDED EXPONENTS
}

\author{
S. V. IVANOV AND A. YU. OL'SHANSKII
}

\begin{abstract}
In 1987, Gromov conjectured that for every non-elementary hyperbolic group $G$ there is an $n=n(G)$ such that the quotient group $G / G^{n}$ is infinite. The article confirms this conjecture. In addition, a description of finite subgroups of $G / G^{n}$ is given, it is proven that the word and conjugacy problem are solvable in $G / G^{n}$ and that $\bigcap_{k=1}^{\infty} G^{k}=\{1\}$. The proofs heavily depend upon prior authors' results on the Gromov conjecture for torsion free hyperbolic groups and on the Burnside problem for periodic groups of even exponents.
\end{abstract}

\section{INTRODUCTION}

Let $\mathcal{A}$ be a finite set of generators for a group $G$. By $|g|=|W|$ denote the length of a shortest word $W$ in the alphabet $\mathcal{A}$ that represents an element $g \in G$.

One of definitions of a hyperbolic group $G$ is given by means of the Gromov product

$$
(g \cdot h)=\frac{1}{2}\left(|g|+|h|-\left|g^{-1} h\right|\right)
$$

as follows: A group $G$ is called hyperbolic $[\mathrm{Gr}]$ if there exists a constant $\delta \geq 0$ such that for every triple $g, h, f \in G$

$$
(g \cdot h) \geq \min ((g \cdot f),(h \cdot f))-\delta .
$$

In turns out $[\mathrm{Gr}],[\mathrm{GH}]$ that the property of being hyperbolic does not depend on a particular generating set $\mathcal{A}$ (but the constant $\delta$ does depend on $\mathcal{A}$ ).

The concept of hyperbolicity has its origins in geometry [Gr]. However, hyperbolic groups deserve the special attention of group theory as well: The class of hyperbolic groups is generic so that almost every (in a definite statistical meaning) finitely presented group is hyperbolic [Gr], [Ol4]. Basic properties of hyperbolic groups are the subject of books [GH], [CDP].

For the free group over the alphabet $\mathcal{A}$ inequality (1) is satisfied with $\delta=0$, because in this case $(g \cdot h)$ is the length of the maximal common beginning of reduced words $g, h$ in $\mathcal{A}$. Similar to free groups, an arbitrary non-elementary hyperbolic group has many homomorphic images (recall a group $\Gamma$ is termed in $[\mathrm{Gr}]$ elementary if $\Gamma$ has a cyclic subgroup of finite index). Discussing an approach to construction of an infinite periodic quotient group $\bar{G}$ of a non-elementary hyperbolic group, Gromov

Received by the editors April 5, 1995.

1991 Mathematics Subject Classification. Primary 20F05, 20F06, 20F32, 20 F50.

The second author was supported in part by Russian Fund for Fundamental Research, Grant 010-15-41, and by International Scientific Foundation, Grant MID 000.

(C)1996 American Mathematical Society 
[Gr] (see also $[\mathrm{GH}],[\mathrm{Ol}]$ ) points out that this approach does not let bound the orders of elements in $\bar{G}$ (and so $\bar{G}$ will not be of finite exponent; recall a group $\bar{G}$ is of exponent $n$ if $x^{n}=1$ for every $x \in G$ ). Nevertheless, Gromov conjectures (see $5.5 \mathrm{E}, 5.5 \mathrm{~F}$ in $[\mathrm{Gr}])$ that it is possible in principle to bound the orders of elements in $\bar{G}$ and obtain $\bar{G}$ of finite exponent $n$.

Thus Gromov suggested a natural expansion of the Burnside problem $[\mathrm{Br}]$ (see also $[\mathrm{Bm}],[\mathrm{Gp}],[\mathrm{MKS}])$ on periodic groups. Indeed, the Burnside problem on the existence of an infinite $m$-generator group of exponent $n$ is obviously equivalent to the problem about the infiniteness of the quotient group $\mathbf{B}(m, n)=\mathbf{F}_{m} / \mathbf{F}_{m}^{n}$, called the free $m$-generator Burnside group of exponent $n$, of a free group $\mathbf{F}_{m}$ of rank $m$ by the subgroup $\mathbf{F}_{m}^{n}$ generated by all $n$th powers of elements of $\mathbf{F}_{m}$. Analogously, for an arbitrary group $G$ the existence of an infinite quotient $\bar{G}$ of exponent $n$ is equivalent to the infiniteness of the quotient $G / G^{n}$ and so the Gromov conjecture amounts to the following.

Conjecture. For every non-elementary hyperbolic group $G$ there exists an $n=$ $n(G)$ such that the quotient group $G / G^{n}$ is infinite.

This Gromov conjecture was confirmed in [Ol5] for torsion free hyperbolic groups (note that in the case when $G$ is a free group the theorem below follows from a well-known theorem due to Novikov and Adian [NA], [Ad], see also [Ol1]):

Theorem ([O15]). For every torsion free non-cyclic hyperbolic group $G$ there exists a positive integer $n(G)$ such that the quotient group $G / G^{n}$ is infinite for any odd $n \geq n(G)$. In addition, $\bigcap_{k=1}^{\infty} G^{k}=\{1\}$.

It is mentioned in [Ol5] that the restriction of being torsion free for $G$ can be somewhat weakened without changing proofs. However, in the general case of a hyperbolic group with torsion there are serious obstacles connected with nonelementary centralizers for elements of $G$ and non-cyclic finite subgroups in $G / G^{n}$ that are essentially the same as those in solving the classical Burnside problem for even exponents $n$.

The recent solution [Iv2] (see also [Iv1] for further references) of the "even" Burnside problem for all sufficiently large even exponents $n$ together with [Ol5] now enables us to prove the Gromov conjecture in its full generality:

Theorem A. For every non-elementary hyperbolic group $G$ there exists a positive even integer $n=n(G)$ such that the following are true:

(a) The quotient group $G / G^{n}$ is infinite.

(b) The word and conjugacy problems are solvable in $G / G^{n}$.

(c) Suppose $n=n_{1} n_{2}$, where $n_{1}$ is odd and $n_{2}$ is a power of 2. Then every finite subgroup of $G / G^{n}$ is isomorphic to an extension of a finite subgroup $K$ of $G$ by a subgroup of the direct product of two groups one of which is a dihedral group of order $2 n_{1}$ and the other is the direct product of several copies of a dihedral group of order $2 n_{2}$.

(d) The subgroup $G^{n}$ is torsion free and $\bigcap_{k=1}^{\infty} G^{k n}=\{1\}$.

Observe that one cannot put the inequality $n \geq n(G)$ in Theorem A as in the Theorem of [Ol5] cited above: consider a hyperbolic group $G$ generated by elements of finite orders $n_{1}, n_{2}, \ldots, n_{m}$. Then $G^{n}=G$ for any $n$ relatively prime with every $n_{1}, n_{2}, \ldots, n_{m}$. 
As a matter of fact, when proving Theorem A, we will encounter several restrictions to be imposed on $n$ and end up with that $n$ must be divisible by $2^{k_{0}+5} n_{0}$ (to say nothing of $n \gg 1$ ), where $\frac{n_{0}}{2}$ is the least common multiple of the exponents of the holomorphs $\operatorname{Hol}(K)$ over all finite subgroups $K$ of $G$ and $k_{0}$ is the minimal integer with $2^{k_{0}-3}>\max |K|$ over all finite subgroups $K$ of $G$.

In particular, we make use of the fact that for any hyperbolic group $G$ there are only finitely many finite groups that are isomorphic to subgroups of $G$. This result seems to be of independent interest and was proved by the second author by showing that there are finitely many conjugacy classes of finite subgroups in $G$ (see Lemma 18 in Sect. 2). We will also need the finiteness of conjugacy classes of subsets in $G$ of a more general type than finite subgroups (Lemma 17).

We also note that Theorem A is quite similar to Theorem A [Iv2] (we choose not to state analogs of Theorems B, C [Iv2], because they are quite similar, more technical, and completely apparent from corresponding subsequent Lemmas).

The proofs in the present article will heavily depend on articles [Ol5], [Iv2]. A considerable part of this article is closely connected with rather long work [Iv2] (actually, we will be reproving almost all Lemmas of [Iv2] with necessary modifications) and we will have to refer the reader to many definitions and arguments in [Iv2]; otherwise, this paper would be unjustifiably long. For the same reason, we refer to many definitions of [Ol5] and [Ol2].

As in [Iv2], information about finite subgroups of $G / G^{n}$ is central and of crucial importance in proofs. Such information is obtained in analogs of Lemmas of Sects. 15-16 [Iv2] (see Sect. 12). In particular, finite subgroups of $G / G^{n}$ are described as in [Iv2] modulo finite subgroups of $G$ (part (b) of Theorem A; see also the analog of Lemma 15.9 in Sect. 12 for a refined version of this description).

Naturally, proofs in this article also make use of various facts of general theory of hyperbolic groups.

Let us discuss the interplay between the theorems above and known problems on the existence of a torsion free subgroup of finite index in every hyperbolic group and on the residual finiteness of every hyperbolic group (the former problem naturally appeared in [Ol5]). Parts (a), (c), (d) of Theorem A would follow from the theorem of [Ol5] provided every hyperbolic group $G$ had a torsion free subgroup $H$ of finite index (which also is hyperbolic [GH]; note it follows from the Selberg theorem [Sl] that every discontinuous group of isometries of hyperbolic space $\mathbb{H}^{k}$ the quotient space by which is compact has a torsion free subgroup of finite index).

Such a subgroup $H$ would exist by Lemma 16 if $G$ were residually finite. This, however, is also still unknown. As a counterexample one could try to consider the groups $G(i)$, where $G(0)=G$, investigated in this article. The groups $G(i)$ are hyperbolic for all $i$ following from the analog of Lemma 21.1 [Iv2] and infinite by Theorem A. Furthermore, making use of Zelmanov's results [Z1]-[Z2] (see also the result of Kostrikin $[\mathrm{Ks}]$ for prime exponents) on the restricted Burnside problem, one could conclude (modulo the classification of finite simple groups) that $G(i)$ is not residually finite for sufficiently large $i$ provided the following (posed in [KN, 10.12] by the second author) had a positive solution.

Problem 1. Is it true that given (prime) $n \gg 1$ and $m>1$ there exists an $N=N(m, n)$ such that every finite group generated by $\mathcal{A}=\left\{a_{1}, \ldots, a_{m}\right\}$ satisfies the identity $x^{n} \equiv 1$ provided $\left(x_{1} \ldots x_{N}\right)^{n}=1$ for arbitrary $x_{k} \in \mathcal{A} \cup\{1\}$ ? 
Problem 1 looks to be intermediate, between the original Burnside problem and its restricted version, and seems to be of independent interest of the discussion above.

It also seems of interest to ask a more general question: Is there a finitely presented group $G$ such that $\bigcap_{k=1}^{\infty} G^{k}=\{1\}$ and $G$ is not residually finite? (If one considers all finitely generated groups, then a counterexample is provided by the free $m$-generator Burnside group $B(m, n)$ of any exponent $n \gg 1, m>1$, following from [Z1]-[Z2] and [Iv2]; for prime $n \gg 1$ this also follows from [Ks] and [NA].)

Taking a more elaborated example of the sequence of groups $G(i)$ (see Ch. 13 [Ol2], Corollary 10 of Theorem 22 in [IO], and Theorem 2 in [Iv3]) we can propose a stronger version of Problem 1 whose negative solution would also imply the existence of a hyperbolic non-residually finite group.

Problem 2. Given prime $p \gg 1$ does there exist an infinite sequence $F_{i}, i=$ $1,2, \ldots$, of finite simple groups such that each $F_{i}$ is generated by some elements $a_{i}$, $b_{i}$ with $a_{i}^{p}=1$ and for any elements $g_{i}, h_{i} \in F_{i}$ which can be represented in $F_{i}$ by words in $\left\{a_{i}^{ \pm 1}, b_{i}^{ \pm 1}\right\}$ of length $<i$ it is true that $g_{i}$ is conjugate to a power of $a_{i}$ and the subgroup generated by $g_{i}, h_{i}$ is either cyclic or the entire $F_{i}$ ?

The basic results of the present article (including a "refined" version of the description of finite subgroups of $G / G^{n}$, see the analog of Lemma 15.9 [Iv2] in Sect. 12) were announced in the lectures of the second author in July 1993 at International conference on combinatorial group theory held on Spetses Island in Greece and in October 1993 at the Meeting of the American Mathematical Society in Texas.

\section{Hyperbolic Preliminaries}

The concepts of a geodesic segment, geodesic polygon in a metric space are assumed to be well known (see $[\mathrm{GH}],[\mathrm{CDP}])$. The Gromov product $(x \cdot y)=(x \cdot y)_{o}$ of two points $x, y$ in a metric space $X$ relative to a point $o \in X$ is defined by the formula

$$
(x \cdot y)_{o}=\frac{1}{2}(|x-o|+|y-o|-|x-y|),
$$

where $|a-b|$ is the distance between points $a$ and $b$.

The hyperbolicity of a metric space $X$ (relative to $o$ and, consequently, relative to any point in $X$, see $[\mathrm{GH}])$ means the existence of a constant $\delta \geq 0$ such that

$$
(x \cdot y)_{o} \geq \min \left((x \cdot z)_{o},(y \cdot z)_{o}\right)-\delta
$$

for arbitrary $x, y, z \in X$.

If this condition is satisfied for a geodesic metric space $X$ ( $X$ is geodesic if any two vertices $a, b$ can be joined by a geodesic segment of the length $|a-b|)$ relative to an arbitrary point $o$, then $X$ will be referred to as a $\delta$-space.

Recall that the standard metric in the Cayley graph $C(G, \mathcal{A})$ of a group $G$ with a system of generators $\mathcal{A}$ is introduced by regarding each edge of $C(G, \mathcal{A})$ isometric to the segment $[0,1]$, and a finitely generated group $G$ is hyperbolic if and only if its Cayley graph is a hyperbolic space ( $\delta$-space).

There is another way to define hyperbolic groups using isoperimetric inequalities. Let $\langle\mathcal{A} \| \mathcal{R}\rangle$ be a presentation of a group $G$ with a finite set of defining relators $\mathcal{R}$. 


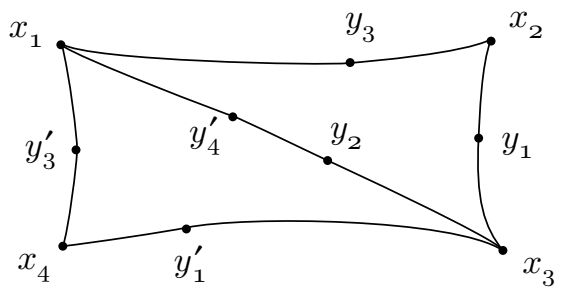

FiguRe 1

Each word $W$ equal to 1 in $G$ can be written in the free group over $\mathcal{A}$ as a product

$$
W=\prod_{i=1}^{t} S_{i} R_{i}^{ \pm 1} S_{i}^{-1},
$$

where $R_{i} \in \mathcal{R}$. The group $G$ is said to satisfy a linear isoperimetric inequality provided the minimal number of factors $t=t(W)$ in the product above is bounded by a linear function of the length $\|W\|$ of a word $W$ (which depends on a particular presentation $\langle\mathcal{A} \| \mathcal{R}\rangle$ of $G$ ). The following statement is well known (see [Gr], [Ly], [GS] for further discussion).

Lemma 1. If a group $G$ is hyperbolic, then $G$ is finitely presented and its every finite presentation satisfies a linear isoperimetric inequality. Conversely, every finitely presented group satisfying a linear isoperimetric inequality is hyperbolic.

The structure of geodesic triangles in a $\delta$-space is specified in [Gr], [GH] as follows.

Lemma 2. For every geodesic triangle $\left[x_{1}, x_{2}, x_{3}\right]$ with vertices $x_{1}, x_{2}, x_{3}$ there are points $y_{i}$ on sides $\left[x_{i-1}, x_{i+1}\right]$ (subscripts $\left.(\bmod 3)\right)$, respectively, such that

$$
\left|x_{i}-y_{i-1}\right|=\left|x_{i}-y_{i+1}\right|=\left(x_{i-1}, x_{i+1}\right)_{x_{i}} \text { and } \operatorname{dist}\left(u,\left[x_{i}, y_{i \pm 1}\right]\right) \leq 4 \delta
$$

for every point $u \in\left[x_{i}, y_{i \mp 1}\right]$.

Lemma 3. Let $\left[x_{1}, x_{2}, x_{3}, x_{4}\right]$ be a geodesic quadrangle in a $\delta$-space with

$$
\left|x_{1}-x_{2}\right|+\left|x_{3}-x_{4}\right| \geq\left|x_{2}-x_{3}\right|+\left|x_{1}-x_{4}\right| .
$$

Then there are points $o, o^{\prime}$ on $\left[x_{1}, x_{2}\right],\left[x_{3}, x_{4}\right]$, respectively, such that $\left|o-o^{\prime}\right| \leq 8 \delta$.

Proof. Let us pick points $y_{1}, y_{2}, y_{3}$ on sides of the triangle $\left[x_{1}, x_{2}, x_{3}\right]$ as described in Lemma 2. Analogously, let $y_{1}^{\prime}, y_{3}^{\prime}, y_{4}^{\prime}$ be points on sides of the triangle $\left[x_{1}, x_{3}, x_{4}\right]$ that has the common side $\left[x_{1}, x_{3}\right]$ with $\left[x_{1}, x_{2}, x_{3}\right]$, see Figure 1.

By Lemma 2 and by the definition of the Gromov product,

$$
\begin{aligned}
& \left|x_{1}-y_{2}\right|-\left|y_{2}-x_{3}\right|=\left|x_{1}-x_{2}\right|-\left|x_{2}-x_{3}\right|, \\
& \left|x_{3}-y_{4}^{\prime}\right|-\left|x_{1}-y_{4}^{\prime}\right|=\left|x_{3}-x_{4}\right|-\left|x_{1}-x_{4}\right| .
\end{aligned}
$$

Summing these equations up and keeping in mind inequality (3), we have

$$
\left|x_{1}-y_{2}\right|-\left|x_{3}-y_{4}^{\prime}\right| \geq\left|x_{1}-y_{4}^{\prime}\right|-\left|y_{2}-x_{3}\right|,
$$

whence $y_{4}^{\prime} \in\left[x_{1}, y_{2}\right]$. 
Once again by Lemma 2 for $u=y_{2}$, we have the inequality

$$
\max \left(\operatorname{dist}\left(y_{2},\left[x_{4}, x_{3}\right]\right), \operatorname{dist}\left(y_{2},\left[x_{1}, x_{2}\right]\right)\right) \leq 4 \delta
$$

that implies our claim.

One can improve the estimate of the closeness of sides $\left[x_{1}, x_{2}\right]$ and $\left[x_{3}, x_{4}\right]$ if one makes inequality (3) stronger. To do this it is convenient to give the definition of $D$-connectedness as follows: Paths $p$ and $q$ are termed $D$-connected provided

$$
\max \left(\left|p_{-}-q_{-}\right|,\left|p_{+}-q_{+}\right|\right) \leq D
$$

where $s_{-}, s_{+}$are the initial, terminal vertex of a path $s$, respectively. Quite similar to Lemma 3, one can prove the following

Lemma 4 ([Ol6]). Let $\left[x_{1}, x_{2}, x_{3}, x_{4}\right]$ be a geodesic quadrangle in a $\delta$-space such that

$$
\left|x_{1}-x_{2}\right| \geq 4 \max \left(\left|x_{1}-x_{4}\right|,\left|x_{2}-x_{3}\right|\right) .
$$

Then there are 8 8 -connected subpaths $p$ and $q$ of the paths $x_{1}-x_{2}$ and $x_{4}-x_{3}$, respectively, such that

$$
\min (|p|,|q|) \geq \frac{7}{20}\left|x_{1}-x_{2}\right|-8 \delta .
$$

Next two lemmas help figure out the structure of geodesic polygons and are proven in [Ol5], see Lemmas 21 and 22. Note the last inequality in Lemma 5 below is immediate from the proof of Lemma 21 [Ol5].

Lemma 5. Let $c \geq 14 \delta, c_{1} \geq 12(c+\delta)$, and a geodesic $n$-gon $\left[x_{1}, \ldots, x_{n}\right]$ with $n \geq 3$ satisfy the following conditions: $\left|x_{i-1}-x_{i}\right|>c_{1}$ for $i=2, \ldots, n$ and $\left(x_{i-2} \cdot x_{i}\right)_{x_{i-1}} \leq c$ for $i=3, \ldots, n$. Then the polygonal line $p=x_{1}-x_{2}-\cdots-x_{n}$ is contained in the closed $2 c$-neighborhood of the side $\left[x_{n}, x_{1}\right]$ and the side $\left[x_{n}, x_{1}\right]$ is contained in the closed $14 \delta$-neighborhood of $p$. In addition, $\left|x_{1}-x_{n}\right|>6(n-1)(c+\delta)$.

Lemma 6. Let the set of all sides of a geodesic $r$-gon $P$ in a $\delta$-space is partitioned into subsets $N_{1}, N_{2}, N_{3}$ the sums of lengths of whose sides are $\sigma_{1}, \sigma_{2}, \sigma_{3}$, respectively, such that $\sigma_{1}>\mathrm{cr}$ and $\sigma_{3}<0.001 \mathrm{cr}$, where $c \geq 3 \delta \cdot 10^{4}$. Then there are distinct sides $p_{1} \in N_{1}, p_{2} \in N_{1} \cup N_{2}$ that contain $14 \delta$-connected segments $q$ and $s$, respectively, of the length $>0.001 \mathrm{c}$.

The following lemmas are well known (see [Gr], [CDP] or Lemmas 1.15-1.17 $[\mathrm{Ol} 6])$.

Lemma 7. Let $g$ be an element of infinite order of a hyperbolic group $G$. Then $g$ is contained in a unique elementary maximal subgroup $E_{G}(g)=E(g) \subseteq G$. In particular, a cyclic subgroup $\langle g\rangle$ of infinite order has a finite index in its centralizer $C_{G}(g)$ in $G$. Furthermore, an element $x \in G$ belongs to the subgroup $E_{G}(g)$ if and only if $x g^{\ell} x^{-1}=g^{ \pm \ell}$ with some $\ell \neq 0$. 
Lemma 8. Let $g$ be an element of infinite order of a hyperbolic group $G$ and an equality $x g^{k} x^{-1}=g^{\ell}$ holds in $G$, where $x \in G, \ell \neq 0$. Then $k= \pm \ell$.

Lemma 7 makes it possible to define the subgroup $E^{+}(g)$ in $E(g)$ that consists of such $x$ 's that $x g^{\ell} x^{-1}=g^{\ell}$ for some $\ell \neq 0$. Since the index of $\langle g\rangle$ in the elementary subgroup $E(g)$ is finite, this $\ell=\ell(x)$ can be chosen the same for all $x$ 's $\in E^{+}(g)$. This means that $E^{+}(g)$ contains a central subgroup $\left\langle g^{\ell}\right\rangle$ of finite index. In this case, by Schur theorem (see Theorem 5.32 [Rt]), all elements of finite order form a subgroup in $E^{+}(g)$ which we will denote by $F(g)$. It follows from the definition of an elementary subgroup that $F(g)$ is finite and the quotient group $E^{+}(g) / F(g)$ is infinite cyclic. Clearly, $E^{+}(g)$ has index $\leq 2$ in $E(g)$. The second coset $E^{-}(g)$ (if any) consists of elements $y$ 's with $y g^{\ell} y^{-1}=g^{\ell}$ for some $\ell \neq 0$. Consequently, the quotient group $E(g) / F(g)$ is either an infinite dihedral or infinite cyclic group.

The length of a path $p$ in the Cayley graph $C(G, \mathcal{A})$ of a group $G$ with a system of generators $\mathcal{A}$ we denote by $\|p\| ;\|W\|$ denotes the length of a word $W$ over $\mathcal{A}$. On the other hand, $|W|,|g|$ will mean the lengths of a shortest word that equals $W$ in $G$, or represents the element $g \in G$, respectively. Analogously, if $p$ is a path in $C(G, \mathcal{A})$, then $|p|$ means the distance from $p_{-}$to $p_{+}$. Clearly, $|p|=|\phi(p)|$, where $\phi(p)$ is the label of $p$.

A word $W$ is termed reduced provided $\|W\|=|W|$. A word $W$ is called cyclically reduced if for any word $V$ the conjugacy of $V$ and $W$ in $G$ implies that $\|W\| \leq\|V\|$.

The following property can be found in [CDP] (pp. 53-54).

Lemma 9. There is a constant $L_{0}=L_{0}(G)$ such that for any two cyclically reduced words $U$ and $V$ that are conjugate in a hyperbolic group $G$ there is a word $X$ with $\|X\|<L_{0}$ and a cyclic permutation $\bar{V}$ of $V$ such that

$$
\bar{V}=X U X^{-1}
$$

in $G$. Next, for any conjugate in $G$ words $A$ and $B$ there is a word $Y$ with $\|Y\|<$ $\ell^{0}(\|A\|+\|B\|)+\ell_{0}$, where $\ell_{0}, \ell^{0}$ depends only on $G$ such that

$$
A=Y B Y^{-1}
$$

in $G$.

Lemma 10. For every hyperbolic group $G$ and every $M>0$ there exists a number $L^{0}=L^{0}(G, M)$ with the following property: if $x, y$ are some elements of infinite order in $G$ with

$$
x=z y z^{-1} \neq 1, \quad \max (|x|,|y|) \leq M
$$

and $z$ has the minimal length in the coset $\langle x\rangle z$, then $|z|<L^{0}$.

Proof. By Lemma 9, there is $L_{1}=L_{1}(M, G)$ such that $x=z_{1} y z_{1}^{-1}$ and $\left|z_{1}\right|<L_{1}$. Then $z z_{1}^{-1}$ belongs to the centralizer $C_{G}(x)$ which, in view of Lemma 7 , is contained in the elementary subgroup $E(x)$. Since the index $|E(x):\langle x\rangle|$ is finite, we have

$$
E(x)=\langle x\rangle s_{1} \cup \cdots \cup\langle x\rangle s_{k}
$$

for some $k$ and so $z z_{1}^{-1}=x^{t} s_{\ell}$ with $\ell \leq k$. By choice of $z$,

$$
|z| \leq\left|s_{\ell} z_{1}\right| \leq\left|s_{\ell}\right|+\left|z_{1}\right| \leq L_{1}+L_{x}
$$

where $L_{x}=\max \left(\left|s_{\ell}\right| \mid 1 \leq \ell \leq k\right)$. 
The right parts of such inequalities are bounded by a common constant in view of the finiteness of the set of all $x$ 's, $y$ 's with $|x|,|y| \leq M$.

A word $W$ (a path $W$ in a $\delta$-space $X$ ) is referred to as a $\left(\kappa_{1}, \kappa_{2}, \theta_{0}\right)$-geodesic, where $\kappa_{1}>0, \kappa_{2} \geq 0, \theta_{0} \geq 0$ provided there is a number $\kappa_{W} \geq \kappa_{1}$ such that for every subword $V$ (subpath $V$, respectively) of $W$ the following holds:

$$
\kappa_{W}\left(\left(1-\theta_{0}\right)\|V\|-\kappa_{2}\right)<|V|<\kappa_{W}\left(\|V\|+\kappa_{2}\right) .
$$

Next lemma is proven in [Ol5], see Lemmas 16 and 28.

Lemma 11. Let $\theta_{0} \leq 0.01$ and $p$ be $a\left(\kappa_{1}, \kappa_{2}, \theta_{0}\right)$-geodesic in a $\delta$-space $X$. Then for every

$$
\lambda>\max \left(5 \kappa_{2}, 700 \kappa_{1}^{-1} \delta\right)
$$

the path $p$ is contained in the $\lambda$-neighborhood of any geodesic segment $\left[p_{-}, p_{+}\right]$(connecting endpoints of $p$ ) which, in its turn, is contained in the $2 \lambda$-neighborhood of the path $p$.

Any subword of a power of some word $B^{ \pm 1}$ is termed a B-periodic word.

The proof of next lemma is given in [Ol5] (see Lemma 27) for a torsion free group $G$. However, that proof is retained for any hyperbolic group $G$ provided one assumes the word $B$ be of infinite order in $G$.

Lemma 12. For every $\delta$-group $G$ and every $\theta_{0}>0$ there exist positive numbers $\kappa_{1}=\kappa_{1}\left(G, \theta_{0}\right)$ and $\kappa_{2}=\kappa_{2}\left(G, \theta_{0}\right)$ such that for every cyclically reduced word $B$ of infinite order in $G$ any $B$-periodic word $V$ is a $\left(\kappa_{1}, \kappa_{2}, \theta_{0}\right)$-geodesic, that is, there is a number $\kappa_{B} \geq \kappa_{1}$ such that

$$
\kappa_{B}\left(\left(1-\theta_{0}\right)\|V\|-\kappa_{2}\right)<|V|<\kappa_{B}\left(\|V\|+\kappa_{2}\right) .
$$

In particular, if $\theta_{0} \leq 1 / 3$ and $\|V\|>2 \kappa_{2}$, then $|V|>\frac{1}{3} \kappa_{1}\|V\|$.

If a cyclically reduced word $B$ called the period (of a periodic $B^{ \pm 1}$-word $V$ ) is sufficiently long, then Lemma $26[\mathrm{Ol}]$ provides a substantially better estimate:

Lemma 13. For every $\delta$-group $G$ and every $\theta_{0}>0$ there exist a number $C=$ $C\left(\theta_{0}, \delta\right)$ such that for every A-periodic word $V$, where $A$ is a cyclically reduced word with $\|A\|>C$, it is true that

$$
|V| \geq\left(1-\theta_{0}\right)\|V\|
$$

Lemma 14. There is a constant $\lambda_{0}=\lambda_{0}(G)$ such that if $W$ is a B-periodic word, where $B$ is a cyclically reduced word of infinite order in $G$, then

$$
|W|>\left|W_{1}\right|+\left|W_{2}\right|-\lambda_{0}
$$

for every factorization $W \equiv W_{1} W_{2}$.

Proof. It suffices to find a common constant to bound from above all Gromov products of the form $\left(V_{1}^{-1} \cdot V_{2}\right)$, where $V_{1}$ and $V_{2}$ are some reduced words that are equal in $G$ to $W_{1}$ and $W_{2}$, respectively. 


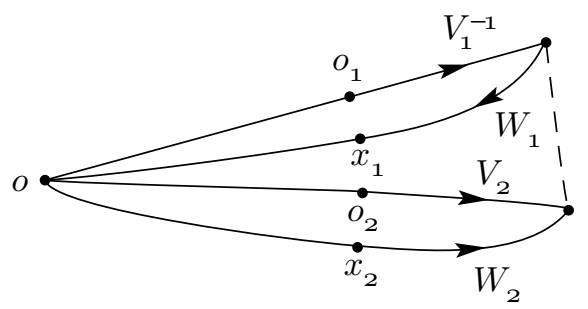

FiguRE 2

Assume that $\left(V_{1}^{-1} \cdot V_{2}\right)>\lambda^{2}$ for some constant $\lambda$ existing by Lemmas 12 and 11 and consider a geodesic triangle in $C(G, \mathcal{A})$ the labels of whose two sides are $V_{1}^{-1}$ and $V_{2}$ (provided we read off the labels starting at the same vertex $o$, see Figure 2).

Lemma 2 enables us to find points $o_{1}$ and $o_{2}$ on these two sides so that

$$
\left|o-o_{1}\right|=\left|o-o_{2}\right|>\lambda^{2}, \quad\left|o_{1}-o_{2}\right| \leq 4 \delta .
$$

Lemma 11 enables us to find points $x_{1}$ and $x_{2}$ on the paths that connect vertices of the triangle and whose labels are $W_{1}^{-1}, W_{2}$, respectively, so that

$$
\max \left(\left|o_{1}-x_{1}\right|,\left|o_{2}-x_{2}\right|\right)<2 \lambda .
$$

Then the label of the path $x_{1}-o-x_{2}$ is a subword of $W$ denoted by $Z$ such that

$$
\|Z\|>2 \lambda^{2}-4 \lambda \text {. }
$$

On the other hand,

$$
|Z|=\left|x_{1}-x_{2}\right|<4 \delta+4 \lambda .
$$

The last two inequalities, however, contradict Lemma 12, since $\lambda=\lambda\left(\kappa_{1}, \kappa_{2}, \delta\right)$ can be chosen arbitrarily large.

Thus, it suffices to put $\lambda_{0}=\lambda^{2}$.

An element $g \in G$ is referred to as $(\Lambda, t)$-periodic provided there are words $U, V, W$ such that one has in the group $G$ that

$$
g=U V W, \quad \max (|U|,|V|) \leq \Lambda,
$$

and $V$ is a $Z$-periodic word, where $Z$ is cyclically reduced, such that $\|V\| \geq t\|Z\|$.

An element $h \in G$ is called $(\Lambda, t)$-aperiodic provided for every factorization $h=$ $h_{1} h_{2} h_{3}$, where $h_{1}, h_{2}, h_{3} \in G$, such that $|h|=\left|h_{1}\right|+\left|h_{2}\right|+\left|h_{3}\right|$ the element $h_{2}$ is not $(\Lambda, t)$-periodic.

The next lemma is established in [Ol5] for torsion free groups, but its proof is retained for any non-elementary $\delta$-group $G$, because according to [Gr], $[\mathrm{GH}]$ every such group contains a free subgroup of rank 2 .

Lemma 15. For every non-elementary hyperbolic group $G$ and every $\Lambda>0$ there is an integer $t>0$ for which the set of $(\Lambda, t)$-aperiodic elements of $G$ is infinite.

Proof. The change incorporated above in the definition of a $(\Lambda, t)$-aperiodic element of $G$ (now $V$ is a $Z$-periodic word of length $\geq t\|Z\|$, not necessarily the $t$ th power like in [Ol5]) makes it possible to repeat arguments of Lemmas 30 and 31 [Ol5]. 


\section{Finite Subgroups of a Hyperbolic Group}

It is derived from Rips's theorem on actions of a hyperbolic group on a finite simplicial complex that there are finitely many conjugacy classes of elements of finite order in $G$ (see Ch. 4 in $[\mathrm{GH}]$ ). A quite different way is used in [Ol5] to bound the length of any cyclically reduced word of finite order in $G$ in [Ol5] (see Lemma 26). In either way, the following is true.

Lemma 16. There is a constant $C_{0}$ depending only upon a hyperbolic group $G$ such that the length of any cyclically reduced word of finite order in $G$ is less than $C_{0}$.

In this paper we will have to use the fact that in any hyperbolic group $G$ there are finitely many conjugacy classes of finite subgroups. Even a stronger statement will also be needed:

Lemma 17. In any hyperbolic group $G$ there are finitely many conjugacy classes of such subsets $M$ 's that $M=M^{-1}$ and both $M$ and $M M$ entirely consist of elements of finite order.

Proof. First let us show that, when proving the lemma, it suffices to consider only finite subsets of $G$. To do this assume that our claim holds for finite subsets and $M \subseteq G$ is an infinite set providing a counterexample. Then $M$ can be represented as a union of finite subsets $M_{i}$ of cardinality $i$ (for which the lemma is true). But we have infinitely many pairwise non-conjugate (due to different cardinalities) finite subsets $M_{i}$, contrary to what was assumed.

So let $M=\left\{g_{1}, \ldots, g_{r}\right\}$ be finite and the maximum

$$
b=\max \left(\left|g_{1}\right|, \ldots,\left|g_{r}\right|\right)
$$

be minimal for all $x M x^{-1}, x \in G$. Then, since $\mathcal{A}$ is finite, it suffices to bound the number $b$ by a constant $K=K(G)$.

Without loss of generality, assume $\delta \geq 1$.

Suppose for some $g=g_{i} \in M$ it is true that

$$
|g|>180 \delta, \quad\left(g \cdot g^{-1}\right)<|g| / 12-\delta .
$$

For arbitrary $n \geq 3$ let us construct a geodesic polygon $\left[x_{1}, x_{2}, \ldots, x_{n}\right]$ in the Cayley graph $C(G, \mathcal{A})$ so that the label $\phi\left(\left[x_{i}, x_{i+1}\right]\right)$ of each side $\left[x_{i}, x_{i+1}\right], i=$ $1,2, \ldots, n-1$, is a reduced word $W$ which represents $g \in G$. By inequality (5), this polygon satisfies the conditions of Lemma 5 with constants $c=|g| / 12-\delta>14 \delta$ and $c_{1}=|g|$. By Lemma 5 ,

$$
\left|g^{n-1}\right|=\left|x_{1}-x_{n}\right|>6(n-1)(c+\delta) \rightarrow \infty,
$$

as $n \rightarrow \infty$. However, $g$ is of finite order. Therefore, if $|g|>180 \delta$, then

$$
\left(g \cdot g^{-1}\right) \geq|g| / 12-\delta .
$$

Now let $g_{i}, g_{j}$ be elements in $M$ with $\left|g_{i}\right|,\left|g_{j}\right|>180 \delta$ and

$$
\left(g_{i}^{-1} \cdot g_{j}\right)<a / 12-3 \delta,
$$




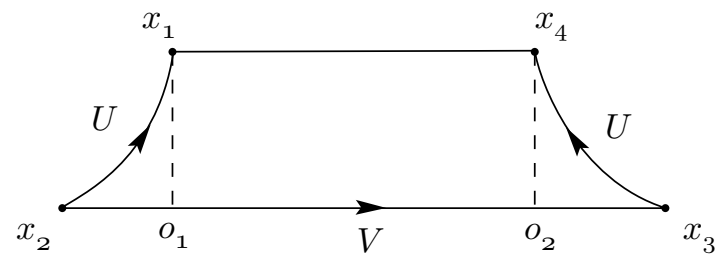

FiguRE 3

where $a=\min \left(\left|g_{i}\right|,\left|g_{j}\right|\right)$. Applying twice inequality (1), we have

$$
\left(g_{i}^{-1} \cdot g_{j}\right)=\left(g_{j} \cdot g_{i}^{-1}\right) \geq \min \left(\left(g_{j} \cdot g_{j}^{-1}\right),\left(g_{j}^{-1} \cdot g_{i}\right),\left(g_{i} \cdot g_{i}^{-1}\right)\right)-2 \delta .
$$

This inequality together with (7) and (6) for $g=g_{i}$ and $g=g_{j}$ yields

$$
\left(g_{j}^{-1} \cdot g_{i}\right)=\left(g_{i} \cdot g_{j}^{-1}\right)<a / 12-\delta .
$$

We can now construct a geodesic $n$-gon $\left[x_{1}, x_{2}, \ldots, x_{n}\right]$ like we did above but so that the label of the polygonal line $x_{1}-x_{2}-\cdots-x_{n}$ is a word that represents the product $g_{i} g_{j} \ldots g_{i} g_{j}(n-1$ is even). Inequalities (7)-(8) show again that the conditions of Lemma 5 are satisfied with the constants $c=a / 12-\delta$ and $c_{1}=a$. This now implies the infiniteness of the order of element $g_{i} g_{j} \in M M$, contrary to the hypothesis. Consequently, it is proven that for every $g_{i}, g_{j} \in M$ with $\min \left(\left|g_{i}\right|,\left|g_{j}\right|\right)>180 \delta$

$$
\left(g_{i}^{-1} \cdot g_{j}\right) \geq a / 12-3 \delta .
$$

Let $g=g_{j} \in M$ and $|g|>180 \delta$. Denote by $U$ the beginning of a reduced word $W$ that represents the element $g$ such

$$
12 \delta-1<|U| \leq 12 \delta .
$$

Also, let $g_{i} \in M$ and $\left|g_{i}\right|>180 \delta$. Then inequality (9) together with Lemma 2 mean the following: if $o \in C(G, \mathcal{A})$ is a point, $o-x$ and $o-y$ are geodesic paths in $C(G, \mathcal{A})$ such that $\phi(o-x) \equiv U$ and $\phi(o-y) \equiv V$, where $V$ is reduced and represents $g_{i}$, then the distance from $x$ to $o-y$ does not exceed $4 \delta$.

Now construct a geodesic quadrangle $\left[x_{1}, x_{2}, x_{3}, x_{4}\right]$ (Figure 3 ) such that

$$
\phi\left(\left[x_{1}, x_{2}\right]\right) \equiv U^{-1}, \phi\left(\left[x_{2}, x_{3}\right]\right) \equiv V, \phi\left(\left[x_{3}, x_{4}\right]\right) \equiv U .
$$

As was pointed out above, there is a point $o_{1} \in\left[x_{2}, x_{3}\right]$ distant at most $4 \delta$ from $x_{1}$. Similarly, $\left|x_{4}-o_{2}\right| \leq 4 \delta$ (if one replaces $g_{i}$ by $g_{i}^{-1}$ ). Therefore,

$$
\min \left(\left|x_{2}-o_{1}\right|,\left|o_{2}-x_{3}\right|\right) \geq|U|-4 \delta>8 \delta-1,
$$

that is, $\left|o_{1}-o_{2}\right|<|V|-2(8 \delta-1)$ and so

$$
\left|x_{1}-x_{4}\right| \leq\left|x_{1}-o_{1}\right|+\left|o_{1}-o_{2}\right|+\left|o_{2}-x_{4}\right|<|V|-16 \delta+2+8 \delta \leq|V|-6 \delta .
$$

Inequality (10) shows that if $M$ contains an element $g$ of length $>180 \delta$, then transition to the set $u^{-1} M u$ (where $u$ is the element of $G$ represented by the word $U$ ) decreases the length of each $g_{i} \in M$ with $\left|g_{i}\right|>180 \delta$ by more than $6 \delta$. 
The length of any other element $g_{i}$ (with $\left|g_{i}\right| \leq 180 \delta$ ) can increase by no more than $2|u| \leq 24 \delta$. This means that if

$$
b=\max \left(\left|g_{1}\right|, \ldots,\left|g_{r}\right|\right) \geq 205 \delta,
$$

then passing to $M^{\prime}=U^{-1} M U$ yields

$$
b^{\prime}=\max (180 \delta+24 \delta, b-6 \delta)<b,
$$

contrary to the choice of $M$.

The proven inequality $b<205 \delta$ completes the proof.

As a corollary, we have

Lemma 18. The set of conjugacy classes of finite subgroups in any hyperbolic $G$ group is finite.

Another corollary of Lemma 17 is the absence of infinite subsets $M$ with $M=$ $M^{-1}$ such that $M$ and $M M$ consist of periodic elements. In particular, there are no infinite periodic subgroups in hyperbolic groups (see also $[\mathrm{Gr}],[\mathrm{GH}]$ ).

Let $g \in G$ be an element of infinite order and $E^{+}(g) \neq E(g)$. Then, as was pointed out in Sect. 1, the quotient group $E(g) / F(g)$ is infinite dihedral. The pre-image in $E(g)$ of an involution of $E(g) / F(g)$ will be called a $g$-involution.

A group $E$ will be referred to as elementary associated with a hyperbolic group $G$ provided $E$ contains a normal finite subgroup $F$ and an element $g$ such that $F \cap\langle g\rangle=\{1\}, F$ is isomorphic to a subgroup of $G$, and either the quotient group $E / F$ is cyclic generated by $g$ or $E / F$ is a dihedral group in which the subgroup $\langle g\rangle$ has index 2, so $E / F=\langle g E, j E\rangle$, where $j E$ is of order 2. Note every finite subgroup $K$ of $G$ is elementary associated with $G(g=1)$.

Lemma 19. Let $G$ be an arbitrary hyperbolic group. Then, in the above notation, there exists an $n_{0}=n_{0}(G)$ so that for every group $E$ elementary associated with $G$ and every $f \in E$ the following equations are true:

$$
(f g)^{n_{0}}=g^{n_{0}}, \quad f g^{n_{0}} f^{-1}=g^{n_{0}}, \quad j g^{n_{0}} j^{-1}=g^{-n_{0}}, \quad(j f)^{n_{0}}=1 .
$$

Proof. By Lemma 18, we can consider the least common multiple $\frac{n_{0}}{2}$ of the minimal exponents of the holomorphs $\operatorname{Hol}(K)$ over all finite subgroups $K$ of $G$. Then the fourth equality in (11) becomes obvious. The first equality holds because of the following chain of equations in $\operatorname{Hol}(K)$ :

$$
\begin{aligned}
(f g)^{n_{0}} & =f\left(g f g^{-1}\right) \ldots\left(g^{n_{0}-1} f g^{-\left(n_{0}-1\right)}\right) g^{n_{0}}=f f^{\alpha_{g}} \ldots f^{\alpha_{g}^{n_{0}-1}} g^{n_{0}} \\
& =\left(f \alpha_{g}\right)^{n_{0}}\left(\alpha_{g}\right)^{-n_{0}} g^{n_{0}}=g^{n_{0}}
\end{aligned}
$$

for $f f^{\alpha_{g}} \ldots f^{\alpha_{g}^{n_{0}-1}}=\left(f \alpha_{g}\right)^{n_{0}}\left(\alpha_{g}\right)^{-n_{0}}=1$ in $\operatorname{Hol}(K)$, where $\alpha_{g}$ is the automorphism of $K$ induced by conjugation by $g$.

The second equality in (11) follows from the first one. The third also follows from the first for $j g j^{-1}=g^{-1} f$ with some $f \in F$. 


\section{Numerical Parameters}

Many definitions and proofs in the sequel will use numerical parameters $\alpha, \beta, \ldots$. The notations for them in [Ol5] and [Iv2] diverge and we choose the notation of [Iv2]. However, in article [Iv2] specific values are assigned to the parameters in order to find a specific estimate for the exponent $n$ (recall $n \geq 2^{48}$ in [Iv2]). This is impossible in the present article for the reason that now the values of the parameters are chosen depending upon a given hyperbolic group $G$. Of course, it would be possible in principle to find them as some explicit functions of $\delta$ for a $\delta$-group $G$. This way would result in some very fast decreasing functions and, in the case of the exponent $n$, in an extremely large and rather complicated function $n=n(\delta)$. In addition, it would definitely make the exposition heavier.

For these reasons we will not be concerned about finding explicit functions $\alpha=$ $\alpha(\delta), \beta=\beta(\delta), \ldots$, and the consistency of the system of all inequalities involved in proofs will be based as in [Ol2], [Ol5] on the least parameter principle (LPP). This means that we arrange all the parameters below according to their "rank": We write $\alpha \succ \beta \succ \gamma \succ \ldots$ ( $\alpha$ is bigger than $\beta, \beta$ is bigger than $\gamma, \ldots)$, meaning that a small number $\beta, \beta>0$, is chosen after $\alpha$, a small number $\gamma, \gamma>0$, is chosen after $\beta$ and so forth. (If $\alpha, \beta, \gamma \ldots$ were to be considered functions of $\delta$, then $\beta$ would be $\overline{\bar{o}}(\alpha), \gamma$ would be $\overline{\bar{o}}(\beta)$ and so on.)

In each inequality of the present paper (as in [Iv2] which will "guide" our exposition), one can find a least parameter. If one considers all the inequalities with the same least parameter, say $\gamma$, then it will be completely clear each time that each of these inequalities (their set is finite) is satisfied provided $\gamma$ is chosen sufficiently small. For example, $4 \gamma(1-\alpha)<\beta^{4}$. (But this is not that trivial sometimes:

$$
\left((1+\beta)(1-\beta)^{-1}(1-\alpha)-1\right)+\gamma<0
$$

not only due to the choice of $\gamma$ but also because, when choosing $\beta$, one can assume that $(1+\beta)(1-\beta)^{-1}<1+3 \beta<1+\alpha / 2$. $)$

Thus, by the least parameter principle, the values of all parameters listed below can be chosen so that the system of all the inequalities involved in proofs is consistent. (The abbreviation "LPP" will sometimes occur as a reminder.)

Let us emphasize that first the constants

$$
L_{0}, \ell^{0}, \ell_{0}, \theta_{0}, \kappa_{1}, \kappa_{2}, \lambda, \lambda_{0}, \Lambda, C_{0}, C_{1}, L, k_{0}, \delta_{0}, n_{0}, C, \delta_{1}, \Omega
$$

(many of which already appeared in Lemmas 9-19) are fixed in the indicated order and only then the parameters in the list (14) below are chosen on the base of LPP.

In connection with consideration of $\delta$-groups in the present article, let us rename the parameter $\delta$ throughout [Iv2] by $\delta_{1}$. Recall $\delta_{1}=1.005$ in [Iv2]. Now $\delta_{1}$ will be much larger (but still $\delta_{1} \ll n$ ). The choice of $\delta_{1}$ depending on $G$ will be specified in Sect. 14.

One more remark: not all parameters in [Iv2] are "close" to 0. For various reasons some of them are close to 1 , to $\frac{1}{2}$, even to infinity. For a unified application of LPP (as in [Ol5], [Ol2]), let us make the following change of parameters (compared with [Iv2]):

$$
\begin{gathered}
\alpha^{\prime}=\alpha-\frac{1}{2}, \eta^{\prime}=1-\eta, \theta^{\prime}=1-\theta, \mu^{\prime}=\mu-1, \\
\rho^{\prime}=1-\rho, \rho_{0}^{\prime}=1-\rho_{0}, \chi^{\prime}=1-\chi, \iota=n^{-1}
\end{gathered}
$$


(in particular, $\iota^{-1}$ is an integer; we will also require $n$ be divisible by $2^{k_{0}+5} n_{0}$, where $n_{0}$ is defined in Lemma 19 and $k_{0}$ is defined in the analog of Lemma 16.2 [Iv2]).

Let us list the numerical parameters according to their "rank" (the order is not alphabetical like in [Ol5], [Ol2] because we follow the notation of [Iv2]):

$$
\begin{aligned}
\mu^{\prime} & \succ \alpha^{\prime} \succ \rho_{0}^{\prime} \succ \rho^{\prime} \succ \eta^{\prime} \succ \zeta \succ \theta^{\prime} \\
& \succ \chi^{\prime} \succ \varepsilon \succ \varepsilon_{0} \succ \beta \succ \omega \succ \xi \succ \gamma \succ \iota .
\end{aligned}
$$

(As a matter of fact, this linear order is not unique.)

In addition to these parameters, proofs in [Iv2] contain several explicitly written down constants (like 30 in Sect. 12 [Iv2], 7 in Sect. 13 [Iv2] etc.). All necessary changes will be indicated in the sequel. The seven integer parameters $K_{1}, \ldots, N$ introduced in Part III [Iv2] (see (17.1) on p. 212 [Iv2]) will also be changed.

\section{Defining Relations and Diagrams}

Let $\mathcal{A}=\left\{a_{1}^{ \pm 1}, \ldots, a_{m}^{ \pm 1}\right\}$ be a system of generators for a given hyperbolic group $G$. It is convenient to assume that all words in the alphabet $\mathcal{A}$ are ordered so that $\|X\|<\|Y\|$ implies $X<Y$. Replacing (compared with [Iv2]) the free group $\mathbf{F}=$ $\mathbf{B}(0)$ by the group $G=G(0)$, we will define by induction on $i$ the groups $G(i)$ that generalize the groups $\mathbf{B}(i)$ in [Iv2]. To do this we need to itroduce (as in [Ol5]) two types of words: "small" and "large". The length of the former words will be bounded by a constant $C=C(G)$.

Recall that in Sect. 1 for every $g \in G$ of infinite order we defined the elementary subgroups $E(g), E^{+}(g)$ and finite $F(g)$.

An element $g \in G$ (or a word $A$ ) of infinite order is called simple in $G=G(0)$ if its coset in $E(g) / F(g)$ generates the cyclic subgroup $E^{+}(g) / F(g)$ and none of the elements of the form $g^{ \pm 1} f$, where $f \in F(g)$, has length $<|g|$.

Consider the set $\mathcal{P}_{i}$ of words $A$ 's that have infinite order in $G(i-1)$ and, if $|A|<C$, then it is required that $A$ is simple in $G(0)$. The period of rank $i$ is the first word in $\mathcal{P}_{i}$ (if any) in the sense of the order introduced above.

The group $G(i)$ is given by the following presentation:

$$
\left\langle\mathcal{A} \| R=1, R \in \mathcal{R}_{0} \cup\left\{A_{1}^{n}, \ldots, A_{i}^{n}\right\}\right\rangle,
$$

where $\mathcal{R}_{0}$ is a set of all relators of the group $G(0)$.

By $i_{0}$ denote the maximal rank for which $\left|A_{i_{0}}\right|<C$.

For all $i \leq i_{0}$ a simple in rank $i$ word $A$ is defined to be simple in $G(0)$ (that is, simple in rank 0). The definition of a simple in rank $i$ word $A$ for $i>i_{0}$ is given as in [Iv2] with the additional condition that $A$ is not conjugate in rank $i$ (i.e. in $G(i))$ to a word of finite order in $G(0)$.

The concept of a diagram of rank $i \geq 0$ (i.e. a diagram over presentation (15) of $G(i)$ ) is defined as in [Ol5] or [Iv2] (but in [Iv2] there are no cells of rank 0 that now correspond to arbitrary relations of $G(0))$. Let us emphasize that it is convenient to include in $\mathcal{R}_{0}$ all the words that are equal 1 in $G(0)$, not excluding trivial relators like $a 1 a^{-1} 1$. (This means that we augment the alphabet $\mathcal{A}$ by the symbol 1 thus obtaining $\mathcal{A}^{1}$, for more details see [O12].)

As in [Ol5], [Ol2], the edges of a diagram $\Delta$ of rank $i$ whose labels are 1 are called 0 -edges, the edges whose labels are letters of $\mathcal{A}$ are termed $\mathcal{A}$-edges. The length of 
a word $W$ over $\mathcal{A}^{1}$ is the number of letters of $\mathcal{A}$ occurring in $W$ (symbols 1 's are not counted) and denoted by $\|W\|$. Analogously, the length of a simplical path $p$ in $\Delta$ is the number of $\mathcal{A}$-edges on $p$ and denoted by $\|p\|$. Clearly, $\|\phi(p)\|=\|p\|$. Exactly as in [Iv2] (but now by means of the length \|\| ), the function $\operatorname{dis}_{p}$ of the distance along a path $p$ is defined.

As in [O15], diagrams are considered together with elementary transformations defined over 0-cells that are called 0-transformations and correspond to replacement of some 0 -cells by other 0 -cells. In particular, if $p$ is a simple path (i.e. $p$ has no selfintersections) in $\Delta$, then, using suitable 0 -transformations (which may be viewed as subdivision of 0-cells into "smaller" 0-cells), one can find a 0-homotopic to $p$ path $\bar{p}$ (this means that only 0-cells can be used to pass from $\bar{p}$ to $p$ ) such that $\phi(\bar{p})=\phi(p)$ in $G$ and $\phi(\bar{p})$ is a reduced word in $G$. As a result, $|p|=\|\bar{p}\|$ by the definition of the length $|p|$.

For an arbitrary diagram $\Delta$ of rank $i$ define its natural map into the Cayley graph $C(G(i), \mathcal{A})$. If $i=0$ this mapping preserves the lengths \|\| and | | of paths. This fact is used in working with diagrams of rank 0. For an arbitrary $i$ a path $p$ is termed 0-geodesic provided $|p|=\|p\|$.

The definitions of compatible sections and reducible pairs of cells (see Sect. 2 [Iv2]) are given only for cells of positive rank $i$ (sections of contours of a diagram cannot have rank 0 ). In the case of a small period $A_{j}$, it is required that the path $t$ in the definition of $j$-compatibility has the label representing an element of the subgroup $F\left(A_{j}\right)$ of $G(0)$. The inequality $|t|<\delta_{1}\left|A_{j}\right|$ in the definition of weak $j$-compatibility is retained.

Recall that the equality of words in $G(i)$ is interpreted by means of a disk (circular) diagram of rank $i$ and conjugacy - by means of annular diagrams of rank $i$ (see [LS], [Ol2], [Iv2], [Ol5]). Let $\Delta$ be an annular diagram over $G$ whose contours are $p$ and $q$. A path $t$ in the annular diagram $\Delta$ is termed cutting if $t$ is simple and $t_{-} \in p, t_{+} \in q\left(\right.$ or $\left.t_{-} \in q, t_{+} \in p\right)$.

Lemma 20. For every $\Lambda>0$ and hyperbolic group $G$ there exists a number $L=$ $L(G, \Lambda)$ with the following property: let $p$ and $q$ be contours of an annular diagram $\Delta$ over $G$ (i.e. $\Delta$ has rank 0 ) such that $|p|,|q|<\Lambda$ and the element $\phi(p)$ has infinite order in $G$. Then there is a cutting path $y$ in $\Delta$ with $|y|<L$.

Proof. This is proved like Lemma 33 [Ol5], but reference to Lemma 14 [Ol5] is replaced by the one to Lemma 10 .

Lemma 21. There exists a number $L=L\left(G, \kappa_{1}, \kappa_{2}\right)$ with the following property: Let each of contours $p, q$ of an annular diagram $\Delta$ over $G$ be factorized into a product of no more than three sections each of which is a $\left(\kappa_{1}, \kappa_{2}, \theta\right)$-geodesic with $\theta \leq 0.01$. Then there is a cutting path $t$ in $\Delta$ with $|y|<L$ provided $\phi(p)$ is of infinite order in $G$. Otherwise, one can claim the existence of an annular diagram $\Delta_{0}$ with the same labels of contours as those of $\Delta$ that has a cutting path $y$ with $|y|<L$.

Proof. We begin by making a remark on the proof of Lemma 34 [Ol5]: In that proof the phrase "the path $q$ (path $p$ ) is located in $(\lambda+8 \delta)$-neighborhood of the path $q^{\prime}$ (path $p^{\prime}$ )" should read " $(2 \lambda+8 \delta)$-neighborhood of any point of the path $q^{\prime}$ (path $\left.p^{\prime}\right)$ contains a point of $q$ ( $p$, respectively)". The latter is immediate from Lemmas $4,28,16[\mathrm{Ol}]$ and still enables us to conclude in proving Lemma 34 [O15] that $\left|y_{3}\right|$ is less than $2 \lambda+8 \delta$ (instead of $2(\lambda+8 \delta)$; after this we proceed as in [Ol5]). 


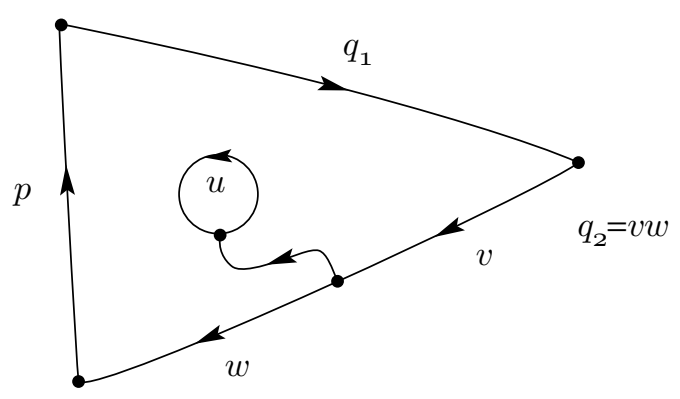

Figure 4

Suppose first that $\phi(p)$ is of infinite order in $G$. Then, taking into account the remark above, we repeat the proof of Lemma 34 [Ol5] just replacing the reference to Lemma 33 [O15] to Lemma 20.

If $\phi(p)$ is of finite order in $G$, then, again as in the proof of Lemma 34 [Ol5], we have either $|y|<L$ or $\min \left(\left|p^{\prime}\right|,\left|q^{\prime}\right|\right)<\Lambda$. After this the annular diagram with contours $p^{\prime}$ and $q^{\prime}$ can be replaced by Lemma 9 by another annular diagram $\Delta_{0}^{\prime}$ so that $\Delta_{0}^{\prime}$ has a path connecting $p_{-}^{\prime}$ and $q_{-}^{\prime}$ (we use the same notation for contours of $\Delta_{0}^{\prime}$ ) which is of length $<2 \ell^{0} \Lambda+\ell_{0}$. It remains to note that this number is less than $L$, because $L$ is chosen after $\ell^{0}, \ell_{0}, \Lambda$.

\section{Simple Elements in a Hyperbolic Group}

Lemma 22. Let $Q \equiv Q_{1} Q_{2}$ be a factorization of a reduced in $G$ word $Q$ such that

$$
|| Q_{1}|-| Q_{2}|| \leq 1
$$

Also suppose the word $P Q$ is of finite order in $G$, where $P$ is reduced in $G$. Then

$$
\left|Q_{2} P Q_{1}\right| \leq|P|+2 C_{0}+4 L .
$$

Proof. By Lemma 21, there exist an annular diagram $\Delta$ over $G$ whose contours are $p q$ and $u$, where $\phi(p)=P, \phi(q)=Q,|u| \leq C_{0}$ by Lemma 16, and a cutting path $t$ in $\Delta$ with $|t|<L$. Assume $t_{-} \in p$. Then, keeping in mind that $Q$ is reduced and cutting along $t$, we have

$$
|Q| \leq|P|+2|t|+|u|<|P|+2 L+C_{0}
$$

which implies (16).

For definiteness assume that $t_{-} \in q_{2}$, where $q=q_{1} q_{2}$ and $\phi\left(q_{2}\right)=Q_{2}$. Let $v$ be the subpath of $q_{2}$ going from $\left(q_{2}\right)_{-}$to $t_{-}$(Figure 4 ).

Since the path $q_{1} v$ is a geodesic, we have

$$
\left|q_{1}\right|+|v| \leq\left|q_{2}\right|-|v|+2|t|+|u|+|p| \leq\left|q_{1}\right|+1-|v|+2|t|+|u|+|p| .
$$

Consequently,

$$
|v| \leq|t|+\frac{1}{2}(|p|+|u|+1) .
$$




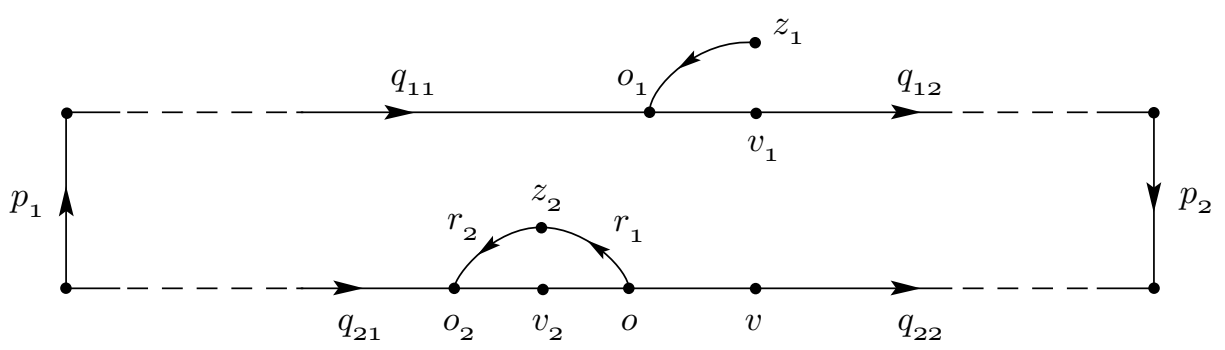

FiguRE 5

Note $Q_{2} P Q_{1}=\phi\left(v t u^{-1} t^{-1} v^{-1}\right)$. Hence,

$$
\left|Q_{2} P Q_{1}\right| \leq 2|v|+2|t|+|u|<|p|+1+2|u|+4|t| \leq|P|+2 C_{0}+4 L ，
$$

as required.

Lemma 23. There exists a constant $\delta_{0}=\delta_{0}(G)$ such that for every $g \in G$ that is of infinite order and has the minimal length in its own conjugacy class the length of any element of $F(g)$ is less than $\delta_{0}$.

Proof. Let the element $g$ be represented by a cyclically reduced word $V$ and $T$ be a reduced word representing an element of $F(g)$. Then, by the definition, we have in $G$ that

$$
T V^{\ell} T^{-1}=V^{\ell}
$$

where $\ell$ can be arbitrarily large (the only restiction on $\ell$ is that $\ell$ is divisible by $\ell_{g}$, where $g^{\ell_{g}}$ is in the center of $F(g)$ ).

Consider a quadrangle $p_{1} q_{1} p_{2} q_{2}$ in the Cayley graph $C(G, \mathcal{A})$ such that

$$
\phi\left(p_{1}\right) \equiv \phi\left(p_{2}\right)^{-1} \equiv T, \quad \phi\left(q_{1}\right) \equiv \phi\left(q_{2}\right)^{-1} \equiv V^{\ell} .
$$

Note that if $\bar{q}_{1}, \bar{q}_{2}$ are geodesics with the same initial and terminal vertices as those of $q_{1}, q_{2}$, respectively, then applying Lemma 4 we can connect a vertex $o^{\prime} \in \bar{q}_{1}$ (or $o^{\prime} \in \bar{q}_{2}$ ) sufficiently distant from both $\left(\bar{q}_{1}\right)_{-},\left(\bar{q}_{1}\right)_{+}$(or from both $\left(\bar{q}_{2}\right)_{-},\left(\bar{q}_{2}\right)_{+}$, respectively) with $\bar{q}_{2}$ (with $\bar{q}_{1}$ ) by a path of length $<8 \delta$. Applying Lemmas 12 and 11, one can make a similar remark about a vertex $o \in q_{1}\left(o \in q_{2}\right)$ sufficiently distant from both $\left(q_{1}\right)_{-},\left(q_{1}\right)_{+}$(or from both $\left(q_{2}\right)_{-},\left(q_{2}\right)_{+}$, respectively): such $o$ can be connected with $q_{2}$ (with $q_{1}$, respectively) by a path shorter than $3 \lambda+8 \delta$.

Let us pick a phase vertex $o_{1} \in q_{1}$ sufficiently distant from both $\left(q_{1}\right)_{-},\left(q_{1}\right)_{+}$ that defines a factorization $q_{1}=q_{11} q_{12}$, where $\phi\left(q_{11}\right) \equiv V^{k}$ and $T V^{k} T^{-1}=V^{k}$ in $G$.

Also, let $o_{2} \in q_{2}$ define the factorization $q_{2}^{-1}=q_{21} q_{22}$ so that $\phi\left(q_{21}\right) \equiv V^{k}$ (Figure 5). Then $\phi\left(o_{1}-o_{2}\right)=T$ in $G$.

As above, let us pick a vertex $o \in q_{2}$ for $o_{1} \in q_{1}$ so that $\left|o-o_{1}\right|<3 \lambda+8 \delta$. For the definiteness assume that $o \in q_{22}$.

In addition, we draw a geodesic path $r$ from $o$ to $o_{2}$ and put $z_{2} \in r$ be the vertex that defines such a factorization $r=r_{1} r_{2}$ of $r$ such that

$$
\left|r_{1}\right| \leq\left|r_{2}\right|, \quad\left|r_{2}\right|-\left|r_{1}\right| \leq 1
$$


By Lemma 22,

$$
\left|\phi\left(r_{2}\right) \phi\left(o_{1}-o\right) \phi\left(r_{1}\right)\right| \leq 3 \lambda+8 \delta+2 C_{0}+4 L .
$$

Denote by $v_{2}$ a closest to $z_{2}$ vertex on the subpath $o-o_{2}$ of $q_{2}$. By Lemmas 12 and $11,\left|z-v_{2}\right|<2 \lambda$.

Let us make another construction. Let us draw a path $\bar{r}$ that ends in $o_{1}$ and has the label $\phi(\bar{r}) \equiv \phi\left(r_{2}\right)$. Put $z_{1}=\bar{r}_{-}$(Figure 5). Then, since $o_{1}, o_{2}$ are phase vertices of $q_{1}, q_{2}^{-1}$ with $V$-periodic labels, one can find a vertex $v_{1} \in q_{12}$ such that $\phi\left(o_{1}-v_{1}\right) \equiv \phi\left(o_{2}-v_{2}\right)$. Clearly, $\left|z_{1}-v_{1}\right|=\left|z_{2}-v_{2}\right|$. Together with (17) this yields

$$
\begin{aligned}
\left|v_{1}-v_{2}\right| & \leq\left|z_{1}-v_{1}\right|+\left|z_{1}-o_{1}-o-z_{2}\right|+\left|z_{2}-v_{2}\right| \\
& \leq 4 \lambda+3 \lambda+8 \delta+2 C_{0}+4 L .
\end{aligned}
$$

At last, pick a vertex $v \in q_{22}$ with $\left|v-v_{1}\right|<3 \lambda+8 \delta$. Considering a geodesic quadrangle $\left[o, v, v_{1}, o_{1}\right]$, we have

$$
\begin{aligned}
|o-v| & \geq\left|o_{1}-v_{1}\right|-2(3 \lambda+8 \delta) \\
& \geq\left|r_{2}\right|-2 \lambda-2(3 \lambda+8 \delta) \geq \frac{1}{2}|r|-8 \lambda-16 \delta .
\end{aligned}
$$

Therefore,

$$
\left\|v_{2}-v\right\|=\left\|v_{2}-o\right\|+\|o-v\| \geq|r|-2 \lambda-8 \lambda-16 \delta .
$$

If $\left|v-v_{2}\right| \leq 2 \kappa_{2}$, then by the last inequality,

$$
|r| \leq 2 \kappa_{2}+10 \lambda+16 \delta .
$$

If $\left|v-v_{2}\right|>2 \kappa_{2}$, then, in view of Lemma 12, we get

$$
\left|v_{2}-v\right|>\frac{1}{3}\left\|v_{2}-v\right\| \geq \frac{1}{3} \kappa_{1}(|r|-10 \lambda-16 \delta) .
$$

However,

$$
\left|v_{1}-v_{2}\right| \geq\left|v-v_{2}\right|-\left|v-v_{1}\right|,
$$

therefore, it follows from (18) that

$$
\frac{1}{3} \kappa_{1}(|r|-10 \lambda-16 \delta)-(3 \lambda+8 \delta)<7 \lambda+8 \delta+2 C_{0}+4 L .
$$

Thus, in either case, we have the following final estimate for $|r|$ :

$$
|r|<\max \left\{2 \kappa_{2}+10 \lambda+16 \delta, 3 \kappa_{1}^{-1}\left(10 \lambda+16 \delta+2 C_{0}+4 L\right)+10 \lambda+16 \delta\right\} .
$$

Since $|T| \leq|r|+\left|o-o_{1}\right|$, it remains to add $3 \lambda+8 \delta$ to the right part of this inequality and denote the obtained sum by $\delta_{0}$.

As in [Ol5], periods of length $<C$ are called small and periods of length $\geq C$ are large.

Lemma 24. (a) Every word $X$ of infinite order in $G(0)$ with $|X|<C$ either has finite order in $G(0)$ or is conjugate in $G(0)$ to an element of $E^{+}(A)$ for some small period $A$. 
(b) Every word $X$ of infinite order in $G(0)$ with $|X| \geq C$ is conjugate in $G(0)$ either to a word of the subgroup $E^{+}(Y)$ for some $Y$ such that $|Y|<|X|$ or to a word of the form $A^{ \pm 1} T$, where $A$ is simple in $G(0)$ and $T \in F(A)$.

Proof. (a) Without loss of generality we may assume $X$ be cyclically reduced and $X$ be conjugate to a word $Z \in E^{+}(A)$ for some simple in $G(0)$ word $A$, following from Lemma 7 and the definition of $E^{+}(A) \subseteq G(0)$.

By Lemma $23, Z=A^{d} T$, where $|T|<\delta_{0}$ and $T \in F(A)$. So it remains to disprove the inequality $|A| \geq C$. By Lemmas 12,21 , there is a cutting path $s$ of length $<L$ in an annular diagram $\Delta$ for the conjugacy of $X$ and $A^{d} T$.

First assume that, when starting moving along the contour of $\Delta$ whose label is $A^{d} T$ at $s_{-}$(or $s_{+}$), one reads off a word of the form

$$
V=A_{2} A^{k} T A^{\ell} A_{1}
$$

where $A_{1} A_{2} \equiv A$ and $k+\ell=d-1$. Since the word $A$ normalizes the normal (in $E(A))$ subgroup $F(A)$, one has $V=A_{2} T_{1} A_{2}^{-1} A_{0}^{d}$, where $T_{1} \in F(A)$ and the word $A_{0} \equiv A_{2} A_{1}$ is simple in $G(0)$ (like $A$ ).

Clearly, that

$$
T_{2} \equiv A_{2} T_{1} A_{2}^{-1} \in A_{2} F(A) A_{2}^{-1}=F\left(A_{0}\right),
$$

whence $\left|T_{2}\right|<\delta_{0}$ following from Lemma 23. Thus (passing, if necessary, to the word $A_{0}$ ), it is possible to assume that $s_{-}$( or $s_{+}$) belongs to the subpath labeled by $T$ of the contour of $\Delta$ labeled by $A^{d} T$. Cutting $\Delta$ along $s$, we obtain a disk diagram for the equation

$$
A^{d}=T^{\prime} S X_{0} S^{-1} T^{\prime \prime}
$$

where $T^{-1} \equiv T^{\prime} T^{\prime \prime}, X_{0}$ is a cyclic permutation of the word $X$, and $S \equiv \phi(s)^{ \pm 1}$. It follows from this equation by Lemma 13 (e.g. for $\theta=0.01$ ) that $\left|A^{d}\right|>0.99|d| C$. On the other hand, the length of the right part of (19) is less than $\delta_{0}+2 L+C$. Inasmuch as $C$ is chosen sufficiently large compared with $\delta_{0}$ and $L$, we have that $|d|=1$. Then $A^{ \pm 1} T$ is conjugate to $X$ and so $|A| \geq C>|X|$. A contradiction to the simplicity of $A$ completes part (a).

(b) This part analogously follows from the inequality $C \gg 2\left(\delta_{0}+2 L\right)$ which, as above, leads to a contradictory inequality

$$
0.99|d||A|<2 L+\delta_{0}+|X|
$$

provided $|d| \geq 2$ and $|A| \geq|X| \geq C$.

What is obvious in Sect. 3 [Iv2] now needs the following explanation:

Lemma 25. A simple in rank $i$ word is also simple in any rank $j<i$. If $\left|A_{i}\right| \geq C$, then every word $X$ of length $<\left|A_{i}\right|$ has finite order in rank $i$ (i.e. in $G(i)$ ).

Proof. This follows from the inductive definition of simple (in ranks $\leq i$ ) words, Lemma 24 , and the finiteness of orders of elements of the subgroups $\mathcal{K}\left(A_{j}\right)$ of $G(i)$ for $j \leq i$ (for the definition of $\mathcal{K}\left(A_{j}\right)$ see Sect. 10 of the present article and Sect. 10 [Iv2]). 


\section{Contiguities And Disconected Arcs}

Smooth sections and their ranks are defined as in [Iv2]. The least parameter principle makes it possible to replace $\omega=10^{-6}$ in [Ol5] by $\alpha^{\prime}$ (that depends on $G$ ). Then Lemma 35 [O15] is restated as follows:

Lemma 26. Suppose $\Delta$ is a tame diagram of rank $i, p_{1}$ and $p_{2}$ are either subpaths of the contour $\partial \Pi$ of a cell $\Pi$ of a positive rank $\leq i$ in $\Delta$, or $p_{1}$ and $p_{2}$ are subpaths of a smooth section $q$ of rank $\leq i$ of the contour $\partial \Delta$ such that $\min \left(\left\|p_{1}\right\|,\left\|p_{2}\right\|\right) \geq \gamma n$. Then there exists $\kappa=\kappa(\Pi)$ or $\kappa=\kappa(q)$ with $\kappa \geq \kappa_{1}$ such that

$$
\left|p_{1}\right| /\left\|p_{1}\right\|=\kappa(1+\tilde{\alpha})
$$

where $|\tilde{\alpha}|<0.9 \alpha^{\prime}$. In addition,

$$
|| p_{1}|/| p_{2}\left|-\left\|p_{1}\right\| /\left\|p_{2}\right\|\right|<\alpha^{\prime} .
$$

The rank and strict rank of a diagram are defined as in [Iv2]. 0-Bonds and 0contigu-

ity subdiagrams, their contiguity degrees and connecting lines are defined as in [Ol5], by means of the parameter $\Lambda$. In the definition of a 0 -bond for the case where edges $e$ and $f$ belong to the same cell $\Pi$ (or to the same section $p$ of a contour), i.e. $\partial \Pi=u e v f$ (or $p=u e v f w$, respectively) it is required that inside the regions with the boundaries $u s^{-1}$ and $v t^{-1}$, where $|s|,|t|<\Lambda$, there are cells of positive rank and/or holes of the diagram.

Keeping this change for 0 -contiguities in mind, we define $k$-bonds and $k$-contiguity subdiagrams by induction on $k$ as in [Iv2]. The definition of degrees of contiguity uses the length \|\| . In particular, $\|\partial \Pi\|$ is the number of non-oriented $\mathcal{A}$-edges on the boundary $\partial \Pi$ of a cell $\Pi$.

We have to be more careful (in comparison with [Iv2], [Ol2]) with the definition of special cells (this remark equally applies to [O15]). Namely, by the definition, there are no special cells in 0 -bonds. For a contiguity subdiagram $\Gamma$ with the standard contour $\partial \Gamma=d_{1}^{1} p_{\Gamma} d_{2}^{2} q_{\Gamma}$ (in the notation of Sect. 2 [Iv2]), a $d_{1}^{1}$-special cell $\left(d_{2}^{2}\right.$-special cell ), or simply a special cell, is a $d_{1}^{1}$-special cell $\left(d_{2}^{2}\right.$-special cell, respectively) of the bond $\mathrm{E}_{1}\left(\mathrm{E}_{2}\right)$. Next, let $\mathrm{E}=d_{1} p_{1} d_{2} q_{1}$ be the standard contour of a bond $\mathrm{E}$, where $d_{1}=c_{2} u_{2}^{-1} b_{1}, \quad d_{2}=b_{2} v_{2}^{-1} c_{1}$ (see Figure 2.4 [Iv2]). Then a $d_{1}$-special cell (or simply a special cell of the bond $\mathrm{E}$ ) is any of the following: the principal cell of E, a $c_{2}$-special cell of the contiguity subdiagram $\Gamma_{2}$, and a $b_{1}$-special cell of the contiguity subdiagram $\Gamma_{1}$. The definition of $d_{2}$-special cells of the bond $\mathrm{E}$ is quite analogous.

In the definition of a tame diagram of rank $i$ (Sect. 2 [Iv2]) the property (D2) now says that any 0 -contiguity subdiagram $\Gamma$ of a cell $\Pi$ to $\Pi$ is such that $\Gamma$ is not contained together with $\Pi$ (and its connecting lines) in a disk (=simply connected) subdiagram of $\Delta$.

As in [Ol5], $|\partial \Pi|$ (or $|\partial \Delta|)$ means the minimum $\left|\partial_{o} \Pi\right|\left(\left|\partial_{o} \Delta\right|\right.$, respectively) over all $o \in \partial \Pi(o \in \partial \Delta)$ of lengths of paths $\partial_{o} \Pi$ 's $\left(\partial_{o} \Delta^{\prime}\right.$ 's $)$ that go once around the contour $\partial \Pi(\partial \Delta$, respectively) starting at a vertex $o \in \partial \Pi(o \in \partial \Delta)$. If $p$ is a contiguity arc of a cell $\Pi$ to something else and $\|p\| /\|\partial \Pi\|=\psi$ is the contiguity degree, then for the ratio $\psi^{\prime}=|p| /|\Pi|$ we have 
Lemma 27. In the above notation, if $\psi \geq \gamma$ then $\psi^{\prime}=\psi(1+2 \tilde{\alpha})$, where $|\tilde{\alpha}|<\alpha^{\prime}$.

Proof. This follows from Lemma 26 (see also Lemma 39 [Ol5]).

In Lemma 3.3 [Iv2], according to Lemma 27, the number $\psi$ in the ratio

$$
(\rho \psi-2 \gamma) /(1-\psi+2 \gamma)
$$

is now replaced by $\psi\left(1-2 \alpha^{\prime}\right)$ and the inequality $\psi<\alpha$ is replaced by $\psi<\alpha+2 \alpha^{\prime}$.

It is now convenient to have two versions of the statement of Lemma 3.4 [Iv2]:

1. $\|\Gamma \wedge \Pi\| /\|\partial \Pi\|<\alpha+2 \alpha^{\prime}$.

2. $|\Gamma \wedge \Pi| /|\partial \Pi|<\alpha$.

The first version follows from the second one and Lemma 27.

The corresponding replacement in proofs of $\alpha$ by $\alpha+2 \alpha^{\prime}$ (when necessary) will not affect inequalities because of an "arbitrary" closeness of $\alpha / \alpha+2 \alpha^{\prime}$ to 1 (LPP).

Taking into account the changes indicated above, the statements of analogs of Lemmas 3.1-3.4 [Iv2] are retained. In their proofs the same corrections as those made in proofs of Lemmas 15.3-15.7 in [Ol5] (up to renaming of parameters indicated in Sect. 3) are made.

Lemma 28. Let $p$ be either a smooth A-periodic with $|A|<C$ section of a contour of a tame diagram $\Delta$ of rank $i$, or $p$ be a section of the contour of a cell $\Pi$ in $\Delta$ that corresponds to a relator of positive rank with a small period. Then any contiguity subdiagram to $p$ is a 0 -contiguity subdiagram.

Proof. By Lemma 3.2 (more accurately, by the analog of Lemma 3.2 [Iv2]) and by Lemma 26, the period $A$ of the smooth section $p$ has length more than

$$
0.94\left(1-0.9 \alpha^{\prime}\right) \kappa_{1} \beta\|\partial \Pi\|>0.9 \beta \kappa_{1} n=0.9 \beta \kappa_{1} \iota^{-1}>C \quad(\mathrm{LPP})
$$

provided some cell has a contiguity subdiagram to this section of degree $\geq \beta$. It remains to refer to the inductive definitions of a bond and a contiguity subdiagram. $\square$

A system $\mathcal{S}$ of contiguity subdiagrams in a diagram $\Delta$ of rank $i$ is referred to as tame if it has properties (E1), (E2) of Sect. 4 [Iv2] ((E3) is now dropped).

Comparing types of different tame systems of contiguity subdiagrams, we pay special attention to the lengths of their contiguity arcs exactly as in Sect. 7 [Ol5]. As in [Ol5], a tame system $\mathcal{S}$ of contiguity subdiagrams of maximal type (among all of the tame systems that can be found in diagrams obtained from $\Delta$ by elementary transformations) will be called distinguished.

Special cells of contiguity subdiagrams in a distinguished system $\mathcal{S}$ are simply called special (relative to $\mathcal{S}$ ). The remaining cells of positive ranks contained in contiguity subdiagrams of $\mathcal{S}$ are termed concealed. At last, the cells of positive ranks not contained in contiguity subdiagrams of $\mathcal{S}$ are referred to as ordinary.

Like in [Ol5] (but unlike [Iv2], [Ol2]), some edges of an ordinary cell may not belong to contiguity arcs of contiguity subdiagrams of a distinguished system $\mathcal{S}$. Each maximal path $p$ (relative to $\|p\|$; perhaps $p$ is cyclic) of the contour of an ordinary cell $\Pi$ all of whose edges have the above property is called a disconnected arc of $\Pi$ (or unbound arc in the terminology of [Ol6]).

The estimating graphs for contiguity subdiagrams are constructed as in [Iv2] (or in [Ol2], [Ol5]). Properties (E4)-(E5) are justified as in [Iv2]. 
As in [Ol5], the sum of lengths of disconnected arcs appears to be relatively small. In comparison with [Ol5], it is necessary to increase the number of contours of the diagrams under consideration up to 3 and the number of sections (smooth or geodesic) of contours of the diagram up to 8 . The worsening in estimates can apparently be compensated by choice of parameters in (14) according to LPP.

Lemma 29. Let $\Delta$ be a tame diagram of rank $i$ with the imposed above restrictions on sections of $\partial \Delta$ and $\mathcal{S}$ be a distinguished system of contiguity subdiagrams in $\Delta$. Then the sum of lengths \|\| of all disconnected arcs in $\Delta$ relative to $\mathcal{S}$ does not exceed $\gamma n m_{\mathcal{S}}$, where $m_{\mathcal{S}}$ is the number of all special and ordinary cells in $\Delta$.

Proof. First note that the proof of Lemma 37 [Ol5] needs the following correction: estimating the number $\sigma_{1}$ one refers to Lemma 12 [Ol5] (corresponding to Lemma 12 of this article). Thus the inequality $\sigma_{1} \geq \frac{1}{4} \alpha \zeta n r$ in [Ol5] should read $\sigma_{1} \geq$ $\left(\frac{1}{4} \alpha k_{1} \zeta n-k_{2}\right) r$. This, however, does not affect the final conclusion. With this only correction we proceed as in the proof of Lemma 38 [Ol5].

\section{Basic Properties of Diagrams of Rank $i$}

The weight of an edge $e$ of an ordinary or special cell $\pi$ (relative to a distinguished system $\mathcal{S}$ in $\Delta$ ) is defined by the formula

$$
\nu(e)=|\partial \pi|^{-1 / 3} .
$$

The condition (F3) after Lemma 5.2 [Iv2] can be dropped inasmuch as the increasing of the total number of sections of contours of $\Delta$ up to 8 does not affect the proofs by virtue of LPP. (The change of the specific constant in Lemma 5.3 from 41 to 50 will not affect the proof of Lemma 5.7 - the only place in [Iv2] that uses Lemma 5.3.)

From now on (if not stated otherwise) we will consider diagrams $\Delta$ 's with properties (F1)-(F2) [Iv2] and, if not stated otherwise, each of sections of $\partial \Delta$ is either smooth or geodesic.

Lemma 30. The sum $J$ of weights of edges of all disconnected arcs in a tame diagram $\Delta$ (relative to a distinguished system $\mathcal{S}$ of contiguity subdiagrams in $\Delta$ ) is less than $\omega \nu(\Delta)$, where $\nu(\Delta)$ is the weight of $\Delta$.

Proof. This repeats the proof of Lemma 40 [Ol5] with replacement of reference to Lemma 38 [Ol5] by reference to Lemma 29. As was noted by Yu.S. Semenov, Lemma 40 [O15] needs the following correction: in its statement and proof, the parameter $\alpha$ must be replaced by a parameter that is smaller than $\gamma$ in [Ol5], for instance, by $\delta$.

Taking into account the additional amount $J$ and its estimate in Lemma 30, one proves main Lemma 5.7 in Sect. 5 as in [Iv2]. Corrections in intermediate inequalities (in Lemmas 5.1-5.5) are the same as those indicated in [Ol5] to the proof of Theorem 16.1 [Ol2] (they are immediate and caused by standard plugging the parameter $\kappa_{1}$ of Lemma 12 in many inequalities).

Like in [O15], the existence of a contiguity subdiagram with some restrictions on degrees of contiguity is now guaranteed not necessarily in $\Delta$ but in a diagram $\Delta^{\prime}$ obtained from $\Delta$ by some elementary transformations. Analogously, the absence of 
contiguity subdiagrams, simple paths etc. is now asserted not only in $\Delta$ but in any diagram $\Delta^{\prime}$ obtained from $\Delta$ by elementary transformations.

The inequality $\rho|q| \leq|p|$ of Lemma 6.1 [Iv2] is retained for disk diagrams. In the annular case, as in [Ol5], this inequality is replaced by $0.9|q|<|p|$ provided $|q| \geq 21 L$. The explanation repeats the one in [Ol5] with reference to Lemma 21 instead of referring to Lemma 34 [Ol5].

The estimate $\rho n\left|A_{k}\right|$ of Lemma 6.2 [Iv2] is replaced, as in [Ol5], by $0.9|\partial \pi|$, where $\pi$ is a cell of rank $k$. (Note that, in general, $\pi$ is not in $\Delta$ and one may replace $|\partial \pi|$ by $\left|A_{k}^{n}\right|$, changing, if necessary, $A_{k}$ by its cyclic permutation when choosing $A_{k}$ in rank $k-1$.)

The analog of Lemma 6.3 is stated as follows.

Lemma 31. Let $\Delta$ be a tame annular diagram of rank $i$ with contours $p$ and $q$, where $p$ is the product of at most three smooth or 0-geodesic sections $p_{1}, p_{2}, p_{3}$ (some of which may be missing) and $q$ is also the product of at most three smooth or 0-geodesic sections $q_{1}, q_{2}, q_{3}$. Then the following are true:

(a) If $\phi(q)$ is of infinite order in $G(0)$, then there is a cutting path $t$ in $\Delta$ with

$$
|t|<\max (L, \zeta(\|p\|+\|q\|)) .
$$

(b) If $\phi(q)$ is of finite order in $G(0)$, then there exists a tame annular diagram $\Delta^{0}$ of rank $i$ the labels of whose contours are $\phi(p), \phi(q)$ such that $\tau\left(\Delta^{0}\right) \leq \tau(\Delta)$ and $\Delta^{0}$ has a cutting path $t$ with the same condition on $|t|$ as in part (a).

Proof. This lemma is analogous to an assertion in [Ol5] (see p. 537) treated there as an analog of Lemma 17.1 [Ol2]. The only difference is that the parameters are now renamed and that, in distinction to [Ol2], [Iv2], $p$ and $q$ consist of several sections. Two parts (a) and (b) come from application of Lemma 21 of Sect. 4 instead of Lemma 34 [Ol5].

In proof of the assertion of [Ol5] there is a gap which was pointed out by Yu.S. Semenov: if the $\theta$-cell $\Pi$ has contiguity subdiagrams only to $p_{1}, p_{2}, p_{3}$ (or only to $\left.q_{1}, q_{2}, q_{3}\right)$, then, proceeding by induction on $\tau(\Delta)$ by we take $\Pi$ out of $\Delta$ and doing so can increase the number of sections of contours in the obtained diagram. This can be fixed as follows.

Making use of 0 -transformations, let us draw geodesic paths $\hat{p}$ and $\hat{q}$ in $\Delta$ and cut off $\Delta$ two subdiagrams of rank 0 with contours $p^{-1} \hat{p}$ and $q^{-1} \hat{q}$. Consider thus obtained annular diagram $\hat{\Delta}$ with contours $\hat{p}$ and $\hat{q}$ (which both consist of a single section). Next, let us pick a $\theta$-cell $\Pi$ in $\hat{\Delta}$. Proceeding by induction on $\tau(\Delta)$, we take $\Pi$ out of $\hat{\Delta}$ and the number of sections of either contour of the resulting annular diagram $\hat{\Delta}_{1}$ will not exceed 3 . This makes it possible to apply the induction hypothesis to $\hat{\Delta}_{1}$.

It remains to estimate the extra term coming from passing from $\Delta$ to $\hat{\Delta}$, that is, the distances from any vertex of the path $\hat{p}$ to $p$ and from any vertex of the path $\hat{q}$ to $q$. If the sides $p_{1}, p_{2}, p_{3}$ of the quadrangle $p_{1} p_{2} p_{3}(\hat{p})^{-1}$ are 0 -geodesics, then by Lemma 2 the distance from any vertex of the path $\hat{p}$ to $p=p_{1} p_{2} p_{3}$ will not exceed $8 \delta$. In the case when some of $p_{1}, p_{2}, p_{3}$ are smooth, it follows from Lemmas 11 and 12 that this distance can additionally increase by at most $2 \lambda$. However, in either case the extra quantity $2(8 \delta+2 \lambda)$ does not affect the final conclusion by virtue of LPP which ensures that all inequalities involved in the inductive step in proofs of any of Lemmas 17.1 [Ol2], 34 [Ol5], 6.3 [Iv2] are still correct. 
In the statement and proof of Lemma 6.4 the factor $n\left|A_{k}\right|$ is replaced by $\left|A_{k}^{n}\right|$ and $|p|$ by the sum of lengths $\left|p_{1}\right|+\ldots$ of sections of $\partial \Delta$ (their number is at most 3). After this Lemma 6.4 is deduced from Lemma 31 (instead of Lemma 6.3 in [Iv2]), because if $\zeta\left(\left|p_{1}\right|+\cdots+|q|\right)<L$ then the diagram $\Delta^{\prime}$ has rank 0 by Lemma 6.2 (note $\zeta \succ \iota=n^{-1}$ by LPP).

In the sequel, the apparent changes of $n\left|A_{k}\right|$ by $\left|A_{k}^{n}\right|$ will only be indicated in statements of lemmas (corresponding changes in proofs will be implied).

The proof of Lemma 6.5 [Iv2] now requires a special consideration of a diagram of rank 0: inasmuch as $\mu>1$, in the case of a geodesic quadrangle Lemma 3 yields the existence of $(8 \delta+2)$-connected edges on paths $p$ and $q$. The reduction of the case of smooth sections to the case of geodesics is performed by standard application of Lemmas 11 and 12 which show the existence of $(4 \lambda+8 \delta+2)$-connected edges on paths $p$ and $q$. Since $\Lambda>4 \lambda+8 \delta+2$, we have a 0 -bond between $p$ and $q$, as required.

The case $r(\Delta)>0$ is handled as in [Iv2] (with apparent corrections in inequalities appearing when one compares lengths with the help of Lemmas 26-27).

\section{Subgroups of Height 0}

We will say that a subgroup $H$ of the group $G(i)$ has height 0 provided each element of $H$ is of height 0 , that is, is conjugate in $G(i)$ to a word that represents in the group $G(0)=G$ an element of finite order.

By the left half of a word $W$ (path $p$ ) we mean the subword $W^{\prime}$ of $W$ (subpath $p^{\prime}$ of $p$, respectively) such that $W \equiv W^{\prime} W^{\prime \prime}\left(p=p^{\prime} p^{\prime \prime}\right)$ and $\left\|W^{\prime}\right\|=[\|W\| / 2]$ $\left(\left\|p^{\prime}\right\|=[\|p\| / 2] ;[]\right.$ is the integer part function). Accordingly, $W^{\prime \prime}\left(p^{\prime \prime}\right)$ is the right half of $W(p)$.

Lemma 32. (a) Let $P, Q$ be reduced in rank $i$ words and $P Q$ conjugate in rank $i$ to a cyclically reduced in rank $i$ word $U$. Then there exists an annular tame diagram $\Delta$ of rank $i$ with contours $p q$ and $u$, where $\phi(p) \equiv P, \phi(q) \equiv Q, \phi(u) \equiv U^{-1}$ such that the end $\left(q_{1}\right)_{+}$of the left half $q_{1}$ of the path $q$ can be joined in $\Delta$ with a vertex on $u$ by a simple path $s$ with

$$
|s|<2|p|+2|u|+3 L+2 \zeta|q| .
$$

(b) Suppose $P$ is a reduced in rank $i$ word, $Q$ is an A-periodic in rank $i$ word, where $A$ is simple in rank $i$ with $|A|>C$. Then there exists an annular tame diagram $\Delta$ of rank $i$ with contours $p q$ and $u$, where $\phi(p) \equiv P, \phi(q) \equiv Q, \phi(u) \equiv U^{-1}$ such that the end $\left(q_{1}\right)_{+}$of the left half $q_{1}$ of the path $q$ can be joined in $\Delta$ with a vertex on $u$ by a simple path $s$ with

$$
|s|<2|p|+2|u|+3 L+3 \theta_{0}|q| .
$$

Proof. (a) Referring to Lemma 31, we find an annular tame diagram $\Delta$ and a cutting path $t$ in $\Delta$ such that

$$
|t|<\max (L, \zeta(|p|+|q|+|u|)) .
$$

Suppose the initial vertex $o=t_{-}$of the path $t$ is on $p$ (we assume $t_{+} \in u$ ). Then, keeping in mind that $Q$ is reduced in rank $i$ and cutting $\Delta$ along $t$ into a disk diagram, we see that

$$
|q| \leq|p|+2|t|+|u| .
$$


This together with inequality (20) yields that

$$
|q|<(1-2 \zeta)^{-1}((1+2 \zeta)(|u|+|p|)+2 L) .
$$

Since the vertices $\left(q_{1}\right)_{+}$and $o$ can be connected by a path, say $s_{0}$, of length $\leq \frac{1}{2}(|p|+|q|)$ (e.g. along $\left.\partial \Delta\right)$, it follows from (20)-(21) for $s=s_{0} t$ that

$$
|s|<2|p|+2|u|+3 L,
$$

as required.

Now assume that $o=t_{-}$sits on the right half $q_{2}$ of $q$ (the case of the left half $q_{1}$ is analogous). Let $q_{2}=v w$ be the decomposition of $q_{2}$ defined by $o$ (Figure 4). Then, since the subpath $q_{1} v$ of $q$ is geodesic, one has

$$
\left|q_{1}\right|+|v| \leq 2|t|+|u|+\left|q_{2}\right|-|v|+|p| .
$$

This together with $\left|q_{2}\right|-\left|q_{1}\right| \leq 1$ implies that

$$
|v| \leq|t|+\frac{1}{2}|u|+\frac{1}{2}|p|+1 \text {. }
$$

Putting $s=v t$, we immediately have the desired estimate for $|s|$ from (22) and (20).

(b) Arguing as above, we pick an annular tame diagram $\Delta$ and a cutting path $t$ in $\Delta$ with (20). If the vertex $o=t_{-}$belongs to $p$, then cutting $\Delta$ along $t$ and applying Lemma 6.1, we have

$$
\rho|q| \leq|p|+2|t|+|u| .
$$

This together with inequality (20) yields that

$$
|q|<(\rho-2 \zeta)^{-1}((1+2 \zeta)(|u|+|p|)+2 L)
$$

and the existence of a desired path $s$ becomes evident from (20) as in part (a).

Now suppose that $o=t_{-} \in q_{2}$ of $q$ (the case $o \in q_{1}$ is analogous). As above, let $q_{2}(\operatorname{dec}, o)=v w($ Figure 4$)$.

Then, by Lemma 6.1 , we have

$$
\rho\left|q_{1} v\right| \leq 2|t|+|u|+\left|q_{2}\right|-|v|+|p| .
$$

Since $q$ is $A$-periodic with $|A|>C$, it follows from Lemma 13 that

$$
\left|q_{1} v\right| \geq\left(1-\theta_{0}\right)\left\|q_{1} v\right\| \geq\left(1-\theta_{0}\right)\left(\left|q_{1}\right|+|v|\right) .
$$

Hence,

$$
\rho\left(1-\theta_{0}\right)\left(\left|q_{1}\right|+|v|\right) \leq 2|t|+|u|+\left|q_{2}\right|-|v|+|p| .
$$

Since $0 \leq\left\|q_{2}\right\|-\left\|q_{1}\right\| \leq 1$ and $\left|q_{1}\right| \geq\left(1-\theta_{0}\right)\left\|q_{1}\right\|$ by Lemma 13 , we get

$$
\left|q_{2}\right|-\left|q_{1}\right| \leq 1+\theta_{0}\left\|q_{1}\right\| \leq 1+\theta_{0}\left(1-\theta_{0}\right)^{-1}\left|q_{1}\right|
$$

following from Lemma 13. Consequently, we further have

$$
\begin{aligned}
\left(\rho\left(1-\theta_{0}\right)+1\right)|v| & \leq 2|t|+|u|+|p|+1+\left(\theta_{0}\left(1-\theta_{0}\right)^{-1}+1-\rho\left(1-\theta_{0}\right)\right)\left|q_{1}\right| \\
& \leq 2|t|+|u|+|p|+1+\left(\theta_{0}\left(1-\theta_{0}\right)^{-1}+1-\rho\left(1-\theta_{0}\right)\right) \frac{1}{2}\left(1-\theta_{0}\right)|q|,
\end{aligned}
$$

because $\left\|q_{1}\right\| \leq \frac{\|q\|}{2}$ and so $\left|q_{1}\right| \leq \frac{1}{2}\left(1-\theta_{0}\right)^{-1}|q|$ by Lemma 13 .

In view of LPP, we finally see that

$$
|v|<1.01|t|+0.51(|u|+|p|)+1+2 \theta_{0}|q| .
$$

By inequality (20), the path $s=v t$ has a desired length. 
Lemma 33. Let $X$ be a reduced in rank $i$ word of height $0, X_{1}$ and $X_{2}$ its left and right halves. Then $X_{2}^{-1} \stackrel{i}{=} X_{1} Z$ for some word $Z$ with

$$
|Z|<4 \zeta|X|+6 L+5 C_{0},
$$

where $C_{0}$ is the constant of Lemma 16.

Proof. Let us apply Lemma 32 (a) to $P \equiv 1$ (i.e. for the empty $P$ ), $Q \equiv X$, and $U$ chosen by Lemma 16 so that $\|U\|<C_{0}$. Cutting the diagram $\Delta$ of Lemma 32(a) along the path $s$, we have a disk diagram for an equation of the form

$$
X_{1} S U_{0} S^{-1} X_{2} \stackrel{i}{=} 1
$$

where $S=\phi(s)^{ \pm 1}$ and $U_{0}$ is a cyclic permutation of the word $U^{-1}$. Setting $Z \equiv$ $S U_{0} S^{-1}$, we have $X_{2}^{-1} \stackrel{i}{=} X_{1} Z$ and $|Z|<C_{0}+2\left(2 C_{0}+3 L+2 \zeta|X|\right)$ following from Lemma 32(a).

By Lemma 17, there is a number $N$ that depends only on $G$ (in fact, $N=N(\delta)$ ) such that each subset $M \subseteq G$ such that $M=M^{-1}$ and $M, M M$ consist of elements of finite order contains less than $N$ elements.

for every $k=1,2, \ldots, N$ define constants $b_{k}$ and $d_{k}$ as follows: For $k=1$ by equations

$$
d_{1}=L+1, \quad b_{1}=C_{0}
$$

and for $k \geq 1$ by formulas

$$
d_{k+1}=4 d_{k}, \quad b_{k+1}=16 b_{k}+40 N\left(C_{0}+2 L\right) .
$$

The following property $\left(\mathrm{P}_{k}\right)$ will be established by for all $k=1,2, \ldots, N$.

$\left(\mathrm{P}_{k}\right)$ Let $X_{1}, \ldots, X_{k}$ be reduced in rank $i$ words of height 0 in rank $i$ such that each product of the form $X_{j} X_{\ell}^{ \pm 1}, 1 \leq j, \ell \leq k$, is also of height 0 in rank $i$. Then there is a word $Y$ the conjugation in rank $i$ by which brings the words $X_{1}, \ldots, X_{k}$ to reduced in rank $i$ words $X_{1}^{\prime}, \ldots, X_{k}^{\prime}$ such that

$$
\sum_{j=1}^{k}\left|X_{j}^{\prime}\right|<b_{k} \quad \text { and } \quad|Y|<d_{k} \sum_{j=1}^{k}\left|X_{j}\right| .
$$

For $k=1$ property $\left(\mathrm{P}_{1}\right)$ follows from Lemmas 16 and 31. Proving property $\left(\mathrm{P}_{k}\right)$, $1 \leq k \leq N$ by induction on $k$, assume that $\left(\mathrm{P}_{k}\right)$ holds for some $k<N$ and consider a set

$$
\mathcal{X}_{k+1}=\left\{X_{1}, \ldots, X_{k}, X_{k+1}\right\}
$$

of reduced in rank $i$ words $X_{1}, \ldots, X_{k}, X_{k+1}$ of height 0 such that every $X_{j} X_{\ell}^{ \pm 1}$, $1 \leq j, \ell \leq k+1$, is also of height 0 .

Making use of property $\left(\mathrm{P}_{k}\right)$ for $\mathcal{X}_{k}=\left\{X_{1}, \ldots, X_{k}\right\}$, we have a conjugate set $\mathcal{X}_{k}^{\prime}=\left\{X_{1}^{\prime}, \ldots, X_{k}^{\prime}\right\}$ and a reduced word $X_{k+1}^{\prime} \stackrel{i}{=} Y X_{k+1} Y^{-1}$. In view of (23),

$$
\left|X_{k+1}^{\prime}\right| \leq\left|X_{k+1}\right|+2 d_{k} \sum_{j=1}^{k}\left|X_{j}\right| .
$$


Lemma 33 yields the the existence of a word $Z$ such that if $X_{k+1}^{\prime} \equiv X_{k+1,1}^{\prime} X_{k+1,2}^{\prime}$ is the decomposition of $X_{k+1}^{\prime}$ into halves then $\left(X_{k+1,2}^{\prime}\right)^{-1} \stackrel{i}{=} X_{k+1,1}^{\prime} Z$ and

$$
|Z|<4 \zeta\left(\left|X_{k+1}\right|+2 d_{k} \sum_{j=1}^{k}\left|X_{j}\right|\right)+6 L+5 C_{0} .
$$

Now let us pass from the set $\mathcal{X}_{k+1}^{\prime}=\left\{X_{1}^{\prime}, \ldots, X_{k}^{\prime}, X_{k+1}^{\prime}\right\}$ to a new set

$$
\mathcal{X}_{k+1}^{\prime \prime}=\left\{X_{1}^{\prime \prime}, \ldots, X_{k}^{\prime \prime}, X_{k+1}^{\prime \prime}\right\}
$$

where $X_{j}^{\prime \prime} \stackrel{i}{=}\left(X_{k+1,1}^{\prime}\right)^{-1} X_{j}^{\prime} X_{k+1,1}^{\prime}$ for $1 \leq j \leq k+1$.

By choice of $Z$, we see $X_{k+1}^{\prime \prime} \stackrel{i}{=} Z^{-1}$, hence the length $\left|X_{k+1}^{\prime \prime}\right|$ is estimated by means of inequality (25).

To estimate $\left|X_{j}^{\prime \prime}\right|$ for $1 \leq j \leq k$, we apply Lemma 32(a) putting $P=X_{j}^{\prime}$, $Q=X_{k+1}^{\prime}$, where $|U|<C_{0}$ by Lemma 16 . Then it follows from Lemma 32(a) with $S \equiv \phi(s)^{ \pm 1}$ that

$$
S U S^{-1} \stackrel{i}{=} X_{k+1,2}^{\prime} X_{j}^{\prime} X_{k+1,1}^{\prime} \stackrel{i}{=} Z^{-1}\left(X_{k+1,1}^{\prime}\right)^{-1} X_{j}^{\prime} X_{k+1,1}^{\prime} \stackrel{i}{=} Z^{-1} X_{j}^{\prime \prime} .
$$

Therefore, in view of (25), we have

$$
\begin{aligned}
\left|X_{j}^{\prime \prime}\right| \leq & |Z|+2|S|+C_{0}<2\left(2\left|X_{j}^{\prime}\right|+2 C_{0}+3 L+2 \zeta\left|X_{k+1}^{\prime}\right|\right) \\
& +4 \zeta\left(\left|X_{k+1}\right|+2 d_{k} \sum_{j=1}^{k}\left|X_{j}\right|\right)+6 L+5 C_{0}+C_{0} .
\end{aligned}
$$

This together with inequalities $(23)-(25)$ and the equation $X_{k+1}^{\prime \prime} \stackrel{i}{=} Z^{-1}$ gives the following estimate:

$$
\begin{aligned}
\sum_{j=1}^{k+1}\left|X_{j}^{\prime \prime}\right|< & 4 b_{k}+10(k+1) C_{0}+12(k+1) L \\
& +4 \zeta(k+1) \cdot 2 d_{k} \sum_{j=1}^{k+1}\left|X_{j}\right|+4 \zeta k \cdot 2 d_{k} \sum_{j=1}^{k+1}\left|X_{j}\right| \\
\leq & 4 b_{k}+10 N C_{0}+12 N L+16 \zeta N d_{k} \sum_{j=1}^{k+1}\left|X_{j}\right| .
\end{aligned}
$$

Since we conjugated the original set $\mathcal{X}_{k+1}$ twice, by $Y$ and by $\left(X_{k+1,1}^{\prime}\right)^{-1}$, and $\left|X_{k+1,1}^{\prime}\right| \leq \frac{1}{2}\left|X_{k+1}^{\prime}\right|$, the last inequality along with (24) and (23) proves the following intermediate statement.

Lemma 34. There is a conjugation in rank $i$ by a word $Y_{1}$ with $\left|Y_{1}\right|<2 d_{k} \sum_{j=1}^{k+1}\left|X_{j}\right|$ that brings the set $\mathcal{X}_{k+1}=\left\{X_{1}, \ldots, X_{k}, X_{k+1}\right\}$ to $\mathcal{X}_{k+1}^{1}=\left\{X_{1}^{1}, \ldots, X_{k}^{1}, X_{k+1}^{1}\right\}$ such that

$$
\sum_{j=1}^{k+1}\left|X_{j}^{1}\right| \leq 4 b_{k}+10 N\left(C_{0}+2 L\right)+16 \zeta N d_{k} \sum_{j=1}^{k+1}\left|X_{j}\right| .
$$

Concluding the inductive step, we prove 
Lemma 35. Property $\left(\mathrm{P}_{k+1}\right)$ holds for $\mathcal{X}_{k+1}$ with $k \leq N$.

Proof. If $\sum_{j=1}^{k+1}\left|X_{j}\right| \geq b_{k+1}$, then, applying Lemma 34, we have from inequality (26) and the definition of numbers $b_{1}, \ldots, b_{k+1}$ that

$$
\sum_{j=1}^{k+1}\left|X_{j}^{1}\right| \leq \frac{1}{2} \sum_{j=1}^{k+1}\left|X_{j}\right|,
$$

because LPP makes it possible to pick $\zeta$ so that $16 \zeta N d_{k} \leq \frac{1}{4}$ for every $k<N$ (say, $\zeta<4^{-N}(L+1)^{-1} N^{-1}$ ). In addition, for the conjugating word $Y_{1}$ we have $\left|Y_{1}\right|<2 d_{k} \sum_{j=1}^{k+1}\left|X_{j}\right|$. Consequently, passing successively from $\mathcal{X}_{k+1}=\mathcal{X}_{k+1}^{0}$ to $\mathcal{X}_{k+1}^{1}$, from $\mathcal{X}_{k+1}^{1}$ to $\mathcal{X}_{k+1}^{2}, \ldots$, we will obtain at some $r$ th step that

$$
\sum_{j=1}^{k+1}\left|X_{j}^{r}\right|<b_{k+1}
$$

and the length of the conjugating word $Y_{0, r}$ (so that $Y_{0, r} \mathcal{X}_{k+1} Y_{0, r}^{-1}=\mathcal{X}_{k+1}^{r}$ ) is estimated as follows:

$$
\begin{aligned}
\left|Y_{0, r}\right| & \leq\left|Y_{1}\right|+\cdots+\left|Y_{r}\right|<2 d_{k}\left(\sum_{j=1}^{k+1}\left|X_{j}\right|\right)\left(1+\frac{1}{2}+\cdots+\frac{1}{2^{r-1}}\right) \\
& <4 d_{k} \sum_{j=1}^{k+1}\left|X_{j}\right|=d_{k+1} \sum_{j=1}^{k+1}\left|X_{j}\right|,
\end{aligned}
$$

as required.

Lemma 36. There are no distinct in rank $i$ words $X_{1}, \ldots, X_{N}$ of height 0 in rank $i\left(N\right.$ is defined in property $\left.\left(\mathrm{P}_{k+1}\right)\right)$ such that every product $X_{j} X_{\ell}^{ \pm 1}, \quad 1 \leq j, \ell \leq N$, is also of height 0 in rank $i$.

Proof. Arguing on the contrary, we apply Lemma 35 to the set $\mathcal{X}_{N}=\left\{X_{1}, \ldots, X_{N}\right\}$ and consider a conjugate set $\mathcal{X}_{N}^{\prime}=\left\{X_{1}^{\prime}, \ldots, X_{N}^{\prime}\right\}$. Due to a suitable choice of $n$ and LPP, we may suppose $\kappa_{1} n>3 b_{N}$. The words $X_{j}^{\prime}\left(X_{\ell}^{\prime}\right)^{ \pm 1}, 1 \leq j, \ell \leq N$, have length $<2 b_{N}<\frac{2}{3} \kappa_{1} n$ and of height 0 in rank $i$. Application of Lemma 6.4 to an annular tame diagram $\Delta$ for conjugacy of such a short word to a word of finite order in rank 0 (and so of length $<C_{0}$ by Lemma 16) yields that $r(\Delta)=0$ as $\rho-2 \zeta>\frac{2}{3}$. Therefore the word $X_{j}^{\prime}\left(X_{\ell}^{\prime}\right)^{ \pm 1}$ is of finite order in rank 0. Analogously, the words $X_{j}^{\prime}, \quad 1 \leq j \leq N$, have finite order in rank 0 . A contradiction to the choice of $N$ completes the proof.

Lemma 37. Every finite subgroup $H$ consisting of words of height 0 in rank $i$ is the isomorphic image (under the natural homomorphism $G(0) \rightarrow G(i)$ ) of a finite subgroup of $G(0)$.

Proof. By Lemma 36, the cardinality $|H|$ of $H$ satisfies $|H|<N$. By Lemma 35, passing from $H$ to a conjugate in rank $i$ subgroup $H_{1}=Y H Y^{-1}$ makes it possible to represent each element of $H_{1}$ by a word $X$ of length $<b_{N}$.

The multiplication table for $H_{1}$ consists of equations $X_{j} X_{k} \stackrel{i}{=} X_{\ell}$, which by Lemmas 6.2 and 26 are true in rank 0 as well, because $3 b_{N}<0.8 \kappa_{1} n$ (LPP). Therefore, the subgroup of $G(0)$ whose elements are the words $Y^{-1} X_{j} Y, 1 \leq j \leq$ $|H|$, will be the pre-image of the subgroup $H$. 


\section{Special Polygons}

The definitions of special 8-gons, $8^{\prime}$-gons, 6-gons, 6'-gons of Sects. 7-8 [Iv2] are essentially retained with the following remarks: in property (G5) instead of equalities $x=b(x=a)$ we now say, " $x$ is 0-homotopic to $b$ " (" $x$ is 0-homotopic to $a$ ", respectively). The same remark applies to property (G6). If the period $A_{k}\left(A_{\ell}\right)$ is small, then instead of $2 \beta^{-1}\left|A_{k}\right|\left(2 \beta^{-1}\left|A_{\ell}\right|\right.$, respectively) in the corresponding inequalities of properties (G4)-(G6) we put the parameter $\Lambda$ which was used in Sect. 6 to introduce 0-bonds. Clearly, all the inequalities in Sects. 7-8 [Iv2] that use these estimates will only improve.

As above, standard changes are made in inequalities involving degrees of contiguity and lengths of contiguity arcs. For example, in Lemma 7.1(c) the inequality

$$
\left|\Gamma_{1} \wedge \Pi\right|+\left|\Gamma_{2} \wedge \Pi\right|<0.8|\partial \Pi|
$$

can be augmented by $\left\|\Gamma_{1} \wedge \Pi\right\|+\left\|\Gamma_{2} \wedge \Pi\right\|<0.8\|\partial \Pi\|$ whose proof uses Lemmas 26 and 27 in standard way.

In Lemma 7.4 [Iv2] the inequality $|\partial \Pi|<19 \beta^{-1} M$ is retained (or, by Lemma 26, $\left.\|\partial \Pi\|<20 \beta^{-1} \kappa_{1}^{-1} M\right)$. In general, in Sects. $7-8$, the lengths || of sections of the contour of $\Delta$ are now compared with $|\partial \Pi|$ (not with $\|\partial \Pi\|$ ) and instead of the perimeter $|\partial \Delta|$ in estimates of Lemmas 8.7-8.8 we use the sum of lengths || of sections of $\Delta$. One can also observe that if $x$ is a smooth $B$-periodic section of $\partial \Delta$ and the cell $\Pi$ has a contiguity to $x$ of degree $\geq \beta$, then $|B|>0.9 \beta \kappa_{1} n$ by Lemmas 3.2 [Iv2] and 26. Therefore, $|x|>\left(1-\theta_{0}\right)\|x\|$ by Lemma 13 (note $0.9 \beta \kappa_{1} n>C\left(\theta_{0}, \delta\right)$ by LPP). Then, in view of arbitrary closeness of $\theta_{0}$ to 0 (in fact, it suffices to put $\theta_{0}=10^{-9}$ ), one can obtain for such $x$ the same esimates for $\|x\|$ as those obtained for $|x|$ in Sects. $7-8$.

The proof of the analog of Lemma 8.5 becomes a somewhat more complicated. Arguing on the contrary, consider a system of 0 -contiguity subdiagrams in $\Delta$ (or in $\Delta^{\prime}$ obtained from $\Delta$ by elementary tranformations) consisting of contiguity subdiagrams of the sections $p$ and $q$ to the other sections of $\partial \Delta$ that is maximal relative to the sum of lengths \|\| of contiguity arcs. Clearly, there are no more than 6 contiguity subdiagrams distinguished this way to the sections $a, b, c, d$. Therefore, the sum of lengths || of their contiguity arcs is less than $8 \beta^{-1} M+12 \Lambda$ following from property (G4) and the definition of 0-contiguity. There are no contiguity subdiagrams between $p$ and $s, p$ and $s, q$ and $r, q$ and $s$, for otherwise, their side arcs would violate properties (G5)-(G6).

Assume now that the length | | of one of contiguity arcs of the contiguity subdiagram $\Gamma$ (if any) between $p$ and $q$ is less than $\frac{1}{2} \beta^{-1} \max \left(\left|A_{k}\right|,\left|A_{\ell}\right|\right)$. Then there is a disconnected arc of $p$ (or $q$ ) of length $>0.1 \beta^{-1} \max \left(\left|A_{k}\right|,\left|A_{\ell}\right|\right)$. Lemma 6 applied to one of the polygons, denoted by $P$, that result from taking $\Gamma$ out of $\Delta$ (more accurately, to a geodesic polygon obtained from $P$ by replacing sides of $P$ by geodesics, see Lemma 11) yields a contradiction because $\beta$ is chosen after $\Lambda$, that is, $\beta^{-1} \gg \Lambda$ (when referring to Lemma 6 , we include the side arcs of contiguity subdiagrams into the set $N_{3}$ ).

Thus it is proven that there is a contiguity subdiagram $\Gamma$ between $p$ and $q$ in $\Delta$ with a contiguity arc of length $\geq \frac{1}{2} \beta^{-1} \max \left(\left|A_{k}\right|,\left|A_{\ell}\right|\right)$. Keeping in mind that side arcs of $\Gamma$ have length $\left\langle\Lambda\right.$ and $\beta^{-1} \gg \Lambda$, we obtain, exactly as in the proof of Lemma 8.6 [Iv2], a contradiction to property (G9) by application to $\Gamma$ of one of Lemmas 18.5(b), 19.1, 17.4(3) in $\operatorname{rank} \max (k, \ell)-1$. 
The assertion of Lemma 8.5 also remains valid after replacing $|p|+|q|$ by $\|p\|+\|q\|$. To see this, we note that if the periods $A_{k}, A_{\ell}$ are large, then this is true due to the fact that $C$ can be chosen sufficiently large and Lemma 26 (since the coefficient $9 \beta^{-1}$ of Lemma 8.5 is taken with a good "margin"). If one of $A_{k}, A_{\ell}$ (or both) is small, then the above argument can be repeated, since the estimates of lengths of corresponding paths $a, b, c, d$ are replaced by $\Lambda$ according to (G4). This, in its turn, makes it possible to use Lemma 12 as $3 \Lambda \kappa_{1}^{-1}<\beta^{-1}$ (LPP). The analog of Lemma 8.5 is now proven.

It is added to the statement of Lemma 9.1(c) that any cell $\pi$ of $\Delta$ such that $|\partial \pi| \geq|\partial \Pi|$, where $r(\Pi)=j=r(\Delta)$, is also the principal cell of a bond of the system $\mathrm{E}_{1}, \ldots, \mathrm{E}_{k}$. In inequality (9.3), the length $\left|\partial \Delta_{t}\right|$ is replaced by the sum of lengths || of sections of $\partial \Delta_{t}$. We also need to add the terms $2 \Lambda, 3 \Lambda, 5 \Lambda$ to the right parts of inequalities (9.1)-(9.3) in the case when $r\left(\pi_{t}\right)=r\left(\pi_{t+1}\right)=0$. (As a matter of fact, this is impossible when one of $p, q$ is a section of the contour of a cell by Lemma 9.2.) In the proof of Lemma 9.1, estimates for 0-bonds of the sums $\left|r_{t}\right|+\left|s_{t}\right|$ are replaced by $2 \Lambda$. As in [Iv2], inequalities (9.1)-(9.3) are proved on the base of Sects. 7-8 (and Lemma 27).

In the proof of Lemma 9.2, instead of $|s|=0$ we now have $|s| \leq \Lambda$, whence $|u| \leq \rho^{-1} \Lambda$. Consequently, the tame (by the induction hypothesis) diagram $\Delta_{0}$ has perimeter $\leq \Lambda\left(1+\rho^{-1}\right)$. Hence $r\left(\Delta_{0}\right)=0$ by Lemma 6.2 (since $\rho n \gg \Lambda\left(1+\rho^{-1}\right)$ ), as required.

In the statements of Lemmas 9.3-9.5 [Iv2] small periods are excluded. (In the case of a small period it follows from Lemmas 28 and 9.1(c) that $r(\Delta)=0$ and there is nothing to prove.)

\section{Elements of Finite Order in the Group $G(i)$}

Applying Lemma 19.4 in a smaller rank $j-1<i$, we define the subgroups $\mathcal{K}\left(A_{j}\right)$ of the group $G(i)$ for $j \leq i$ as in [Iv2]:

$$
\mathcal{K}\left(A_{j}\right)=\mathcal{G}\left(A_{j}\right) /\left\langle A_{j}^{n}\right\rangle .
$$

Lemma 18.5 and the definition of Sect. 19 [Iv2] enable us to introduce in $\mathcal{K}\left(A_{j}\right)$ the finite subgroup $\mathcal{F}\left(A_{j}\right)$ and $\mathcal{F}\left(A_{j}\right)$-involutions. By Lemmas 19.2 and 40 (applied in rank $j-1<i$ ), the finite subgroup $\mathcal{O}\left(\mathcal{F}\left(A_{j}\right)\right.$ ) (which will also be denoted by $\left.\mathcal{O}\left(A_{j}\right)\right)$ of height 0 is normal in $\mathcal{K}\left(A_{j}\right)$ and, by Lemma 37 , is the isomorphic image of a finite subgroup of $G=G(0)$.

The statement of Lemma 10.1 [Iv2] is retained.

In part (a) of Lemma 10.2 a second option emerges: the word $W$ can be of height 0 in rank $i$ (that is, in $G(i)$ ). In part (b) of Lemma 10.2, we add that a word of the form $A_{j}^{k} F$, where $j>0$ and $0<k<n$, is not conjugate in rank $i$ to a word of finite order in $G(0)$ (that is, $A_{j}^{k} F$ is not of height 0 in rank $i$ ) and that the conjugacy in rank $i$ of two words of finite order in $G(0)$ implies their conjugacy in $G(0)$.

To prove part (a), arguing on the contrary, we assume $W$ be not of height 0 and just repeat the argument of [Iv2] leading to a contradiction.

In proving part (b), like in many other lemmas, an equality of the form $\left|A^{\ell}\right|=$ $\ell|A|$ now is not true and, to estimate $\left|A^{\ell}\right|$, we have to apply Lemmas 26,27 or 12 provided $A$ is a period of some rank or a simple in some rank word. (In this particular case, it is quite sufficient to use a rough estimate of Lemma 12.) 
To the proof of Lemma 10.2(b) we also add if two words $V$ and $W$ are of finite order in $G(0)$, then Lemma 16 enables us to assume that $|V|,|W|<C_{0}$. By Lemma 18 , we have $X V X^{-1} W \stackrel{i}{=} 1$, where $2|X|+|V|+|W|<2 L+4 C_{0}$. Then, by Lemmas 6.2 and 27, this equation holds in rank 0 as well (LPP). At last, considering the case where the word $A_{k}^{j} F$ is of height 0 in rank $i$ and arguing as in [Iv2], we obtain an equality of the form

$$
\phi(b) A_{j}^{\ell m_{1}} F_{3} \phi(c) U \stackrel{i}{=} 1,
$$

where $\frac{n}{4} \leq\left|\ell m_{1}\right|<\frac{n}{2}$ and $|U|<C_{0}$ (as $U$ is a cyclically reduced word of finite order in $G(0))$. By the definitions,

$$
|b|+\left|F_{3}\right|+|c|+|U|<2\left(\zeta n+1.1+\delta_{1}+C_{0}\right)\left|A_{j_{1}}\right| .
$$

On the other hand, by Lemma $12,\left|A_{j}^{\ell m_{1}}\right|>\frac{1}{12} \kappa_{1} n$ as $\left|\ell m_{1}\right| \geq \frac{n}{4}$. Applying Lemma 6.1, we have

$$
\left(\frac{1}{12} \kappa_{1} \rho-2 \zeta\right) n<2\left(1.1+\delta_{1}+C_{0}\right) .
$$

An obvious contradiction (LPP) completes the argument.

Lemma 10.2 together with the definition of words of height 0 enables us to define, as in [Iv2], the height of any word of finite order in rank $i$ (that is, in $G(i)$ ).

In the statement of Lemma 10.4 that calculates the order $d$ of a word $W$ in rank $i$ we make the following corrections.

The words $V_{A_{j}}$ and $S_{A_{j}}$ emerge according with Lemma 19.5 as in [Iv2] only if $\mathcal{F}\left(A_{j}\right)$ is of positive height. In the case when the height of $\mathcal{F}\left(A_{j}\right)$ is 0 , the number $d_{2}$ is defined differently: $d_{2}$ is the least positive integer such that $\left(A_{j}^{k} F\right)^{d_{2}} \stackrel{j-1}{=} A_{j}^{k d_{2}}$. Next, in part (b), we now say that the order of the coset of $A_{j}^{k}$ in the quotient group $\mathcal{K}\left(A_{j}\right) / \mathcal{O}\left(A_{j}\right)$ is a positive power of 2 that divides $n_{2}$. In part (c), instead of $F=1$ we now assert that $F \in \mathcal{O}\left(A_{j}\right)$.

To prove this analog of Lemma 10.4, we add in the proof of part (a) that if $h\left(\mathcal{F}\left(A_{j}\right)\right)=0$, then, by Lemma $37, \mathcal{F}\left(A_{j}\right)$ is the isomorphic image of a subgroup of $G(0)$. Hence, in the case of height 0 , the conjugations by powers of $A_{j}$ in the chain of equalities (10.12) [Iv2] are now replaced by actions of the automorphisms of $\mathcal{F}\left(A_{j}\right)$ induced by conjugations by powers of $A_{j}$ (instead of conjugations by powers of $V_{A_{j}}$ like in [Iv2]). Then the number $d_{2}$ will be a divisor of $n$ by Lemma 19, since the exponent $n$ was chosen divisible by $n_{0}=n_{0}(G)$. The proof of part (c) is retained but instead of the finite subgroup $\left\langle V_{A_{j}}^{k}, V_{A_{j}}^{k} F\right\rangle$ we consider the finite subgroup

$$
\mathcal{H}=\left\langle V_{A_{j}}^{k}, V_{A_{j}}^{k} F, \mathcal{F}\left(A_{j}\right)\right\rangle \subseteq G(j-1)
$$

and its quotient group $\mathcal{H} / \mathcal{O}(\mathcal{H})$ (see the definition of $\mathcal{O}(\mathcal{H})$ in Sect. 12 for $j-1<i$ ). Then, as in [Iv2], references to Lemmas $15.9,18.5$ (a) yield that $F \in \mathcal{O}(\mathcal{H})$. Since $h(\mathcal{H})=h\left(\mathcal{F}\left(A_{j}\right)\right)$ by choice of $V_{A_{j}}$ and Lemmas 15.7-15.8 (applied in rank $\left.j-1<i\right)$, it follows from Lemma $40(\mathrm{f})$ (applied in rank $j-1<i$ ) that

$$
\mathcal{O}\left(A_{j}\right)=\mathcal{O}\left(\mathcal{F}\left(A_{j}\right)\right)=\mathcal{F}\left(A_{j}\right) \cap \mathcal{O}(\mathcal{H})
$$

It becomes obvious that $F \in \mathcal{O}\left(A_{j}\right)$ and Lemma 10.4 is proven. 
In Lemma 10.5, in addition to removal of reducible pairs of cells we allow elementary 0-transformations (this is a standard feature when working with 0-cells).

It is additionally required in the hypothesis of Lemma 10.7 that in any annular diagram $\Delta^{\prime}$ with contours $p^{\prime}=q^{\prime} t^{\prime}$ and $s^{\prime}$, where $\phi\left(s^{\prime}\right)=\phi(s), \quad \phi\left(p^{\prime}\right)=\phi(p)$, $\phi\left(q^{\prime}\right)=\phi(q)$ the contour $p^{\prime}$ is strictly quasismooth provided $\tau\left(\Delta^{\prime}\right) \leq \tau(\Delta)$. (This condition is to make reference to Lemma 31 in the proof valid. It is not restrictive, since we can always pick $\Delta$ with this condition.)

The coefficient 7 in the right part of the inequality of Lemma 10.8(b) becomes $7 \delta_{1} \kappa_{1}^{-1}$, because $|A| \geq \kappa_{1}\left(0.99\left\|A^{k}\right\|-\kappa_{2}\right)$ by Lemma 12 . In the proof of Lemma 10.8(b), we encounter an extra term $2 L$ caused by the new version of Lemma 10.7. This, however, is insignificant, since in the process of arguing we have that

$$
\left|A_{j_{1}}\right|>0.1\left(\delta_{1}+L\right)^{-1} n\left|A_{m}\right| \gg L \quad(\mathrm{LPP}) .
$$

\section{AuXiLiary Diagrams}

In statements and proofs of Lemmas $11.1-11.3$ the quantity $n\left|A_{j}\right|$ is replaced by $\left|\partial \Pi_{1}\right|\left(\left|\partial \Pi_{2}\right|\right)$ or $\left|A_{j}^{n}\right|$. Similar changes are made in the definition of close $j$ projections and in Lemmas 11.4, 12.1-12.3, 13.1, 13.1.1.

In the definition of a $T$-diagram, the inequality $|b|<30\left|A_{j}\right|$ becomes $|b|<$ $30 \delta_{1} \kappa_{1}^{-1}\left|A_{j}\right|$.

The proof of Lemma 12.1 [Iv2] becomes a more complicated in the case $r(\Delta)=0$. First consider a disjoint system of contiguity subdiagrams with the maximal sum of lengths \|\| of contiguity arcs between sections $q, c_{1}, c_{2}, b$. As in the proof of Lemma 38 [O15], we derive the conclusion that the sum of lengths \|\| of disconnected arcs relative to the system is less than $\gamma n$. Next, since the chosen system cannot contain more than three contiguity subdiagrams of the section $q$ to $c_{1}$ and $c_{2}$ (without loss of generality, one may assume $c_{1}, c_{2}$ be geodesics in $\Delta$ ), the sum of lengths || of contiguity arcs of these contiguity subdiagrams to $q$ is less than

$$
6 \Lambda+\left|c_{1}\right|+\left|c_{2}\right|<6 \Lambda+4 \beta^{-1}\left|A_{j}\right| .
$$

Analogously, the length || of the contiguity arc of $q$ to $b$ is less than

$$
2 \Lambda+|b|<2 \Lambda+30 \delta_{1} \kappa_{1}^{-1}\left|A_{j}\right|
$$

following from property (T5).

As follows from property (T2) and Lemma 19.1 in rank $j-1<i$, the sum of lengths \|\| of contiguity arcs of $q$ to $q$ is less than $2 \cdot 484 \Omega\left|A_{j}\right|$.

As a result we have:

$$
|q|<\gamma n+8 \Lambda+\left(4 \beta^{-1} \cdot 30 \delta_{1} \kappa_{1}^{-1}+968 \Omega\right)\left|A_{j}\right|,
$$

It remains to observe that the right part is less than $0.04\left|A_{j}^{n}\right|$, since $\left|A_{j}^{n}\right|>$ $\kappa_{1}\left(1-\alpha^{\prime}\right) n\left|A_{j}\right|$ by Lemma 26 .

In the definition of a $U$-diagram, we again replace $n\left|A_{j}\right|$ by $\left|A_{j}^{n}\right|$. In additition, the coefficient 30 in property (U3) reads $30 \delta_{1} \kappa_{1}^{-1}$ and the word $\phi(e)$ may now be of height 0 (but the inequality $\left(\frac{n}{2}+2 \delta_{1}\right)\left|A_{m}\right|<30 \delta_{1} \kappa_{1}^{-1}\left|A_{j}\right|$ is required only when $m>0)$. 
Analogously, in the statement of Lemma 13.1 we put $35 \delta_{1} \kappa_{1}^{-1}$ and $7 \delta_{1} \kappa_{1}^{-1}$ instead of 35 and 7, respectively, and the word $\phi(e)$ may be of height 0 .

The divergence div is defined as in [Iv2] by means of the length \|\| .

In Lemma 13.2 we also put $7 \delta_{1} \kappa_{1}^{-1}$ instead of the coefficient 7 and allow that the words

$$
\phi(e(1)), \phi(e(2)), \phi(e(1)) \phi(e(2))
$$

may now be of height 0 . The same change is made to the coefficient 14 in Lemma 13.3 and $n\left|A_{j}\right|$ is replaced by $\left|\partial \Pi^{*}\right|$.

The changes in statements of Lemmas 13.4, 13.4.1 are quite similar to those in Lemmas 13.1-13.3.

The analogs of Lemmas 14.2-14.4 will not be used in the present article and so we do not discuss them.

Considering $c$-regular systems (Sect. 14 [Iv2]), we impose the same restrictions on the number of contours of a diagram $\Delta$ and on the number of sections of contours of $\Delta$ as those are introduced in Sect. 6 above. The only difference is that we now consider contiguity subdiagrams between sections of contours of $\Delta$ as well.

A system of contiguity subdiagrams in $\Delta$ is termed c-tame if it has property (V1) of Sect. 14 [Iv2]. A c-distinguished system, special, concealed, and ordinary cells relative to it are defined as in Sect. 6 without the prefix "c-".

Proving the analog of Lemma 38 [Ol5] (and considering $\partial \Delta_{i}$, see the notation of [Ol5]), we regard disconnected arcs of sections of $\Delta$ as arcs of the form (I). As a result, we have that the sum of lengths \|\| of all disconnected arcs relative to a $c$-distinguished system of contiguity subdiagrams in $\Delta$ is at most $\omega n\left(m_{\mathcal{S}}+1\right)$, where $m_{\mathcal{S}}$ is the number of all special and ordinary cells in $\Delta$. (The extra term $\omega n$ comes from disconnected arcs of sections of $\partial \Delta$.)

Properties (V4)-(V5) for a $c$-distinguished system $\mathcal{S}$ are proved as in [Iv2].

In the definition of a $W$-diagram it is assumed that $j_{1}>0$ and possible equality $j_{2}=0\left(j_{3}=0\right)$ means the finiteness of order in $G(0)$ of a non-empty (meaning \|\|$>0)$ cyclically reduced word $\phi\left(p_{1}\right)\left(\phi\left(p_{2}\right)\right.$, respectively).

In condition (W2) of the definition of a $W$-diagram instead of $\left|q_{1}\right|,\left|q_{2}\right|,\left|q_{3}\right|$ we put $\left\|q_{1}\right\|,\left\|q_{2}\right\|,\left\|q_{3}\right\|$, respectively.

In the notation of [Iv2], we now distinguish 6 sections of contours of a $W$-diagram $\Delta$ of rank $i: q_{1}, t_{1}, q_{2}, t_{2}, q_{3}, t_{3}$.

The distribution of weights $\nu$ of edges in $\Delta$ (see Sect. 14 [Iv2]) is retained except for that a non-zero weight is now assigned only to edges of the smooth part $q_{1}$ of $p_{1}$ among all edges of $\partial \Delta$ : if $e \in \partial \Delta$ is an edge and $e \in q_{1}$ then $\nu(e)=\left|A_{j_{1}}^{n}\right|^{-1 / 3}$, otherwise, $\nu(e)=0$. In view of property (W2), Lemma 30 of Sect. 6 still holds for $W$-diagrams.

Note that in the case $j_{2}=0$ or $j_{3}=0$ the estimates below can only improve.

In the statement of Lemma 14.7, the section $p$ is now a section of a contour of $\Delta$ or the contour of a cell in $\Delta$.

Lemma 38. Let $\Gamma$ be a contiguity subdiagram of a c-distiguished system in a Wdiagram $\Delta$ defined between $t$, where $t$ is one of $t_{1}, t_{2}, t_{3}$ and a section of $\partial \Delta$. Then $\nu(\Gamma)<7 \omega \nu\left(q_{1}\right)$.

Proof. Let $\pi$ be the principal cell of one of the (at most two) bonds that define $\Gamma$ and assume $t=t_{1}$. Standard comparison of the length of $\pi \wedge \Gamma$ with $\left|t_{1}\right|$ gives

$$
|\pi \wedge \Gamma|<\left|t_{1}\right|+2 \gamma|\partial \pi| \text {. }
$$


By Lemma 18.5(c) in rank $j_{1}-1<i$, we have $\left|t_{1}\right|<\delta_{1}\left|A_{j_{1}}\right|$ and, by Lemma 27 and the definition of a bond, $|\pi \wedge \Gamma|<\beta\left(1-2 \alpha^{\prime}\right)|\partial \pi|$. Therefore, it follows from (27) that

$$
|\partial \pi|<\left(\beta\left(1-2 \alpha^{\prime}\right)-2 \gamma\right)^{-1} \delta_{1}\left|A_{j_{1}}\right|<\frac{3}{2} \beta^{-1} \delta_{1}\left|A_{j_{1}}\right| .
$$

Hence, by Lemma 26,

$$
\begin{aligned}
\nu(\pi) & <\left(1+2 \alpha^{\prime}\right) \kappa_{1}^{-1}\left(\frac{3}{2} \beta^{-1} \delta_{1}\left|A_{j_{1}}\right|\right)^{2 / 3} \\
& <\omega\left(\frac{1}{2} \kappa_{1} n\left|A_{j_{1}}\right|\right)^{2 / 3}<\omega\left(\left|A_{j_{1}}^{n}\right|\right)^{2 / 3} .
\end{aligned}
$$

Arguing in standard way to estimate the sum of weights of all special cells in $\Gamma$, we have the extra coefficient $2 \omega(1-2 \omega)^{-1}$ and so

$$
\nu(\Gamma)<2 \omega(1-2 \omega)^{-1}\left(\left|A_{j_{1}}^{n}\right|\right)^{2 / 3}<7 \omega \nu\left(q_{1}\right),
$$

following from condition (W2).

If $t=t_{2}$ or $t=t_{3}$, then analogous estimates hold, because $\left|A_{j_{2}}\right|,\left|A_{j_{3}}\right| \leq\left|A_{j_{1}}\right|$ for $j_{2}, j_{3} \leq j_{1}$ by the definition of periods.

Now let us estimate the weight $\nu\left(\Gamma \wedge q_{1}\right)$, where $\Gamma$ is defined as in the proof of Lemma 38.

It follows from inequality (28) and the definition of a bond that

$$
\left|\Gamma \wedge q_{1}\right|<2 \max \left((1+2 \gamma) \frac{3}{2} \beta^{-1} \delta_{1}\left|A_{j_{1}}\right|, \Lambda\right)+\delta_{1}\left|A_{j_{1}}\right| .
$$

In view of property (W2), Lemma 12, inequality (29), and LPP, we have

$$
\left\|\Gamma \wedge q_{1}\right\|<\omega\left\|q_{1}\right\|
$$

whence we see that

$$
\nu\left(\Gamma \wedge q_{1}\right)<\omega \nu\left(q_{1}\right) .
$$

Keeping in mind that all contiguity arcs not belonging to $q_{1}$ have weight 0 , we can now repeat the sequence of estimates of Sect. 14, making use of Lemma 38 , inequality (30), and the estimate $\omega n\left(m_{\mathcal{S}}+1\right)$ for the sum of weights of all disconnected arcs in $\Delta$ obtained above. By virtue of insignificance of all extra terms (LPP), we still have the same inequality as that in Lemma 14.13 [Iv2] for the sum of weights of outer edges in $\Delta$ (but the quantity $n\left|A_{j_{1}}\right|$ in the proof of Lemma 14.13 now reads $\left.\left|A_{j_{1}}^{n}\right|\right)$.

\section{Finite Subgroups of the Group $G(i)$}

The height $h(\mathcal{G})$ of a finite subgroup $\mathcal{G}$ of the group $G(i)$ is defined as in Sect. 15 [Iv2]. Recall that a simple description of finite subgroups of $G(i)$ of height 0 was given in Lemma 37.

In the definition of a tame subgroup we add that a subgroup of height 0 is also tame.

In the statement of Lemma 15.1, the case $k=h(\mathcal{G})=0$ is now allowed as well. If $k=0$, then Claim 1 of Lemma 15.1 simply says that $h(\langle\mathcal{G}, W\rangle)=0$. 
In proofs of Lemmas $15.1,15.4$ the coefficient 7 must be replaced by $7 \delta_{1} \kappa_{1}^{-1}$ in accordance with the correction made in Lemma 10.8. In the case $h(\mathcal{G})=0$, passing to a conjugate subgroup if necessary, one can apply Lemma 18 to bound the lengths of all words representing elements in $\mathcal{G}$ by some constant $C_{1}=C_{1}(G)<\delta_{1} \kappa_{1}^{-1}$.

In the statement of Lemma 15.2, the elements of odd order of $\mathcal{G}$ have now the form indicated in [Iv2] modulo the subgroup $\mathcal{O}\left(A_{h}\right)$ (see the definition of $\mathcal{O}\left(A_{h}\right)$ in rank $h-1<i$ preceding Lemma 40). In other words, each element of odd order of $\mathcal{G}$ is of the form $S A_{h}^{n_{2} \ell} T S^{-1}$ (the notation of [Iv2]), where $T \in \mathcal{O}\left(A_{h}\right)$.

Proof of the analog of Lemma 15.2. Repeating the proof of Lemma 15.2 [Iv2], we first suppose $h(\mathcal{G})=k>0$ (for otherwise the subgroup $\mathcal{G}$ is tame by the definition). The assumption of Case 1 now is that $\mathcal{G}$ contains an element of odd order and positive height. This case is handled as Case 1 in [Iv2] with following corrections. In accordance with Lemma $10.4(\mathrm{c})$, we replace the word $A_{j_{2}}^{\ell_{2}}$ by $A_{j_{2}}^{\ell_{2}} T_{2}$, where $T_{2} \in$ $\mathcal{O}\left(A_{j_{2}}\right)$, and the equation $U=1$ by the inclusion $U \in \mathcal{O}\left(A_{k}\right)$. For the same reason, in the proof of Lemma 15.2.2, the word $A_{k}^{\ell_{1}}$ is replaced by $A_{k}^{\ell_{1}} T_{1}$, where $T_{1} \in \mathcal{O}\left(A_{k}\right)$.

To complete the proof of Lemma 15.2 we need the following

Lemma 39. Suppose a finite subgroup $\mathcal{G} \subseteq G(i)$ is generated by some words $X_{1}, \ldots, X_{s}$ that are of odd order and height 0 in rank $i$. Then $h(\mathcal{G})=0$.

Proof. Arguing on the contrary, assume $h(\mathcal{G})=k>0$. Then, as in the beginning of the proof of Lemma 15.2 [Iv2], conjugating $\mathcal{G}$ if necessary, we pick in $\mathcal{G}$ a word of the form $W_{1} \stackrel{i}{=} A_{k}^{\ell_{1}} T_{1}$, where $T_{1} \in \mathcal{F}\left(A_{i}\right)$ and

$$
\frac{n}{3} \leq\left|\ell_{1}\right| \leq \frac{2 n}{3}
$$

In addition, we may assume that $W_{1}$ (with the above properties) is minimal relative to the length $\|V\|_{\mathcal{X}}$ of a shortest word $V$ in the alphabet $\mathcal{X}=\left\{X_{1}^{ \pm 1}, \ldots, X_{s}^{ \pm 1}\right\}$ such that $W_{1} \stackrel{i}{=} V(\mathcal{X})$. This property obviously implies the existence of a product $W_{1} \stackrel{i}{=} W X$, where $X \in \mathcal{X}, W \in \mathcal{G}$, and the word $W$ is not conjugate in rank $i$ to a product of the form $A_{k}^{\ell} T$, where $T \in \mathcal{F}\left(A_{i}\right)$ and $\frac{n}{3} \leq|\ell| \leq \frac{2 n}{3}$.

Clearly, $X^{-1} \stackrel{i}{=} X_{2} B X_{2}^{-1}$, where $B$ is a word of finite order in $G(0)$, and $W^{-1} \stackrel{i}{=}$ $X_{3} C X_{3}^{-1}$, where either $C$ is a word of finite order in $G(0)$ (when $h(W)=0$ ), or $C \equiv A_{j_{3}}^{\ell_{3}} T_{3}$, where $j_{3} \leq k, 0<\ell_{3}<n$, and $T_{3} \in \mathcal{F}\left(A_{j_{3}}\right)\left(\right.$ when $\left.h(W)=j_{3}>0\right)$.

As in the proof of Lemma 15.2.1, making use of the equation $W_{1} X^{-1} W^{-1} \stackrel{i}{=} 1$, we construct diagrams $\Delta$ and $\Delta_{0}$ with two holes and, as there, (observing now that now $X^{-1}$ is of odd order) show that $j_{3}=k$.

Keeping the notation of Lemma 15.2 .1 , we further have $W_{1} V_{2} V_{3} \stackrel{i}{=} 1$, where $V_{2}, V_{3} \in \mathcal{K}\left(A_{k}\right)$ are conjugate in rank $i$ to $X^{-1}, W^{-1}$, respectively. Since $V_{2}$ is of odd order and height 0 , it follows from Lemma $10.4(\mathrm{c})$ that $V_{2} \in \mathcal{O}\left(A_{k}\right) \subseteq \mathcal{F}\left(A_{k}\right)$. Then

$$
V_{3}^{-1} \stackrel{i}{=} W_{1} V_{2} \stackrel{i}{=} A_{k}^{\ell_{1}} T^{\prime}
$$

where $T^{\prime} \in \mathcal{F}\left(A_{k}\right)$. Since $W$ is conjugate to $V_{3}^{-1}$, we have a contradiction that completes the proof of Lemma 39.

Coming back to the proof of Lemma 15.2, first consider the case where all the elements $X_{1}, \ldots, X_{s}$ of $\mathcal{G}$ that have finite odd order are of height 0 . 
If the subgroup $\mathcal{H}=\left\langle X_{1}, \ldots, X_{s}\right\rangle$ is the entire $\mathcal{G}$, then $h(\mathcal{G})=0$ by Lemma 39 and so $\mathcal{G}$ is tame by the definition. Assume $\mathcal{H} \neq \mathcal{G}$. Since $\mathcal{H}$ is obviously normal in $\mathcal{G}$, we can consider the quotient group $\mathcal{G} / \mathcal{H}$. Since $\mathcal{G} / \mathcal{H}$ obviously contains no non-trivial elements of odd order, there is a normal subgroup $\mathcal{N}$ of index 2 in $\mathcal{G} / \mathcal{H}$. Now that $\mathcal{G}$ is tame can be proven by repeating the argument of Case 2 of the proof of Lemma 15.2.

The claim on elements of odd order in $\mathcal{G}$ is proven as in [Iv2].

The analog of Lemma 15.2 is completely proven.

In the statements of Lemmas 15.3-15.4, we require that $k>0$.

Let us give the definition of a 0-part $\mathcal{O}(\mathcal{G})$ of a finite subgroup $\mathcal{G}$ of $G(i)$. Proceeding by induction on $h(\mathcal{G})$, put $\mathcal{O}(\mathcal{G})=\mathcal{G}$ provided $h(\mathcal{G})=0$. Let $h(\mathcal{G})=j>0$. By Lemma 15.2, we have for some word $X$ that $X^{-1} \mathcal{G} X \subseteq \mathcal{K}\left(A_{j}\right)$. By the definition of the subgroup $\mathcal{F}\left(A_{j}\right)$ (see Sect. 18 [Iv2] in rank $j-1<i$ ), we have $h\left(\mathcal{F}\left(A_{j}\right)\right)<j$. Therefore, the subgroup $\mathcal{O}\left(\mathcal{F}\left(A_{j}\right)\right)$, denoted also by $\mathcal{O}\left(A_{j}\right)$, is already defined. Then we set

$$
\mathcal{O}(\mathcal{G}) \stackrel{i}{=} \mathcal{G} \cap X \mathcal{O}\left(A_{j}\right) X^{-1} .
$$

Basic properties of 0-parts of finite subgroups of $G(i)$ are collected in the lemma below.

Lemma 40. Let $\mathcal{G}$ be a finite subgroup of $G(i)$ with $h(\mathcal{G})=j$ and $\mathcal{O}(\mathcal{G})$ a 0-part of $\mathcal{G}$. The the following are true:

(a) The subgroup $\mathcal{O}(\mathcal{G})$ is well defined (i.e. does not depend on choice of the conjugating word $X$ in its definition).

(b) $\mathcal{O}\left(W \mathcal{G} W^{-1}\right) \stackrel{i}{=} W \mathcal{O}(\mathcal{G}) W^{-1}$ for every word $W$.

(c) The subgroup $\mathcal{O}(\mathcal{G})$ is normal in $\mathcal{G}$ and even in the normalizer of the subgroup $\mathcal{G}$ in $G(i)$.

(d) $h(\mathcal{O}(\mathcal{G}))=0$.

(e) In the case $j \leq k \leq i$, the subgroup $\mathcal{G}$ has a finite pre-image $\overline{\mathcal{G}}$ in $G(k)$ under the natural epimorphism $\Xi: G(k) \rightarrow G(i)$; any such pre-image $\overline{\mathcal{G}}$ is injectively mapped by $\Xi$ onto $\mathcal{G}$ so that $\Xi(\mathcal{O}(\overline{\mathcal{G}}))=\mathcal{O}(\mathcal{G})$.

(f) If $\mathcal{H} \subseteq \mathcal{G}$ is a subgroup and $h(\mathcal{H})=h(\mathcal{G})$, then $\mathcal{O}(\mathcal{H}) \stackrel{i}{=} \mathcal{H} \cap \mathcal{O}(\mathcal{G})$.

Proof. (a) Let $j>0$ and the subgroup $\mathcal{G}$ be also contained in a subgroup $Y \mathcal{K}\left(A_{j}\right) Y^{-1}$ of the group $G(i)$, that is, both

$$
\mathcal{G}_{1} \stackrel{i}{=} X^{-1} \mathcal{G} X \quad \text { and } \quad \mathcal{G}_{2} \stackrel{i}{=} Y^{-1} \mathcal{G} Y
$$

be contained in $\mathcal{K}\left(A_{j}\right)$. Then $\mathcal{G}_{2} \stackrel{i}{=} Z^{-1} \mathcal{G}_{1} Z$ with $Z \stackrel{i}{=} X^{-1} Y$. Inasmuch as $j>0$, Lemma 15.4 applies to this conjugacy and yields the existence of a word $S_{Z} \in$ $\mathcal{K}\left(A_{j}\right)$ such that $S_{Z} Z^{-1} \in C_{G(i)}\left(\mathcal{G}_{1}\right)$. This property along with the normality of the subgroup $\mathcal{O}\left(\mathcal{F}\left(A_{j}\right)\right)=\mathcal{O}\left(A_{j}\right)$ (which follows from parts (c), (e) of Lemma 40 applied in rank $\left.h\left(\mathcal{F}\left(A_{j}\right)\right) \leq j-1<i\right)$, gives us that

$$
\begin{aligned}
\mathcal{G} \cap X \mathcal{O}\left(A_{j}\right) X^{-1} & \stackrel{i}{=} \mathcal{G} \cap X\left(Z S_{Z}^{-1}\right) \mathcal{O}\left(A_{j}\right)\left(S_{Z} Z^{-1}\right) X^{-1} \\
& \stackrel{i}{=} \mathcal{G} \cap Y S_{Z}^{-1} \mathcal{O}\left(A_{j}\right) S_{Z} Y^{-1} \stackrel{i}{=} \mathcal{G} \cap Y \mathcal{O}\left(A_{j}\right) Y^{-1}
\end{aligned}
$$

as required. 
(b) By the definition,

$$
\mathcal{O}\left(W \mathcal{G} W^{-1}\right) \stackrel{i}{=} W \mathcal{G} W^{-1} \cap W X \mathcal{O}\left(A_{j}\right) X^{-1} W^{-1} \stackrel{i}{=} W \mathcal{O}(\mathcal{G}) W^{-1} .
$$

(c) This is immediate from part (b).

(d) This is easy to see from the inductive definition of the 0 -part.

(e) This follows from Lemmas 15.2, 10.1, 10.2, 37 and the definition of the 0-part.

(f) This is obvious.

Lemma 15.5 is stated as in [Iv2] modulo the 0-part $\mathcal{O}(\mathcal{G})$ : It now asserts that $\mathcal{G} / \mathcal{O}(\mathcal{G})$ is a 2 -group. To prove it, we proceed as in [Iv2] regarding all claims on elements of $\mathcal{G}$ modulo $\mathcal{O}(\mathcal{G})$.

In this article we do not consider the case of odd exponent $n$ and so there is no analog of Lemma 15.6.

The statements of Lemmas 15.7-15.8 contain the additional condition: $h\left(\mathcal{F}\left(A_{j}\right)\right)$ $>0$ (otherwise, they would not be true). Their proofs are retained.

Lemma 15.9 is stated as in [Iv2] modulo the 0 -part $\mathcal{O}(\mathcal{G})$ with one extra remark (the case of odd exponent $n$ is removed). In its statement the group $\mathcal{G}$ is replaced by $\mathcal{G} / \mathcal{O}(\mathcal{G})$ and it is additionally asserted that $\mathcal{G}^{n_{0}}$ has no non-trivial elements of height 0. In particular, $\mathcal{G}^{n_{0}} \cap \mathcal{O}(\mathcal{G})=\{1\} \quad\left(n_{0}\right.$ is defined in Lemma 19 of Sect. 2) and so $\mathcal{G}$ is isomorphic to a subgroup of $(\mathcal{G} / \mathcal{O}(\mathcal{G})) \times\left(\mathcal{G} / \mathcal{G}^{n_{0}}\right)$, or to a subgroup of the direct product of dihedral groups as in [Iv2] and a group of exponent $n_{0}$.

We can also propose a second version of Lemma 15.9 (that provides a somewhat more detailed description of finite subgroups of $G(i)$ and so seems worth mentioning here but it will not be used in the sequel): any finite subgroup $\mathcal{G}$ of $G(i)$ with $h(\mathcal{G})=j$ is isomorphic to a subgroup of the direct product $\mathbb{D}(2 n) \times \mathbb{D}\left(2 n_{2}\right)^{j} \times E$, where $\mathbb{D}(2 n)$ denotes a dihedral group of order $2 n$ and $E$ is an elementary associated with $G(0)$ group (defined in Sect. 2).

1. Proving the analog of Lemma 15.9, we first show by induction on $h(\mathcal{G})$ that $\mathcal{G}^{n_{0}}$ has no non-trivial elements of height 0.

The basis step for $h(\mathcal{G})=0$ is obvious by the definition of $n_{0}$. Let $h(\mathcal{G})=j>0$. By Lemmas 15.2 and $40(\mathrm{f})$, without loss of generality we can assume that $\mathcal{G}=$ $\mathcal{K}\left(A_{j}\right)$. Let $X \in \mathcal{K}\left(A_{j}\right)$ be a word of height 0 and

$$
X \stackrel{i}{=} Y_{1}^{n_{0}} \ldots Y_{\ell}^{n_{0}}
$$

where $Y_{1}^{n_{0}}, \ldots, Y_{\ell}^{n_{0}} \in \mathcal{K}\left(A_{j}\right)$.

If $h\left(\mathcal{F}\left(A_{j}\right)\right)=0$, then it follows from Lemmas 37, 19 and the definitions of $\mathcal{K}\left(A_{j}\right)$ and $n_{0}$ that $Y^{n_{0}} \in\left\langle A_{j}\right\rangle$ for every $Y \in \mathcal{K}\left(A_{j}\right)$. Hence $X \stackrel{i}{=} 1$ for $h(X)=0$.

Assume $h\left(\mathcal{F}\left(A_{j}\right)\right)>0$. By Lemma 10.1 and the definition of the height, we have that either $X \in \mathcal{F}\left(A_{j}\right)$ or $X$ is an $\mathcal{F}\left(A_{j}\right)$-involution.

In the first case, application of Lemma 15.7(e) or Lemma 15.8(d) to $\mathcal{K}\left(A_{j}\right)$ shows that $X \stackrel{i}{=} 1$ following from the induction hypothesis applied to $\mathcal{T}$ (note $h(\mathcal{T})<j$ by Lemmas 15.7-15.8).

Since $n_{0}$ is even, it is immediate from definitions of Sects. 18-19 of [Iv2] that $Y^{n_{0}} \in\left\langle\mathcal{F}\left(A_{j}\right), A_{j}\right\rangle$ for every $Y \in \mathcal{K}\left(A_{j}\right)$. Hence $X$ cannot be an $\mathcal{F}\left(A_{j}\right)$-involution and the second case is impossible.

That the group $\mathcal{G}$ may be replaced by $\mathcal{G} / \mathcal{O}(\mathcal{G})$ in Lemma 15.9 is proved quite similar to [Iv2] with an extra reference to Lemma 40(f): 
Proceeding by induction on $h(\mathcal{G})=j$, we note that $h(\mathcal{G})=0$ implies $\mathcal{O}(\mathcal{G})=\mathcal{G}$ and there is nothing left to prove.

Suppose $j>0$. If $h\left(\mathcal{F}\left(A_{j}\right)\right)=0$, then the claim is obvious from Lemma 10.1(a).

Assume $h\left(\mathcal{F}\left(A_{j}\right)\right)>0$. It is easy to see from Lemmas 15.2, 40(f) and the definition of the 0 -part that, without loss of generality, we can suppose $\mathcal{G}=\mathcal{K}\left(A_{j}\right)$. Recall that $\mathcal{O}\left(\mathcal{K}\left(A_{j}\right)\right)=\mathcal{O}\left(\mathcal{F}\left(A_{j}\right)\right)=\mathcal{O}\left(A_{j}\right)$ (by the definition of the 0-part). Hence, by Lemma 40(f), the group $\mathcal{T}$ of Lemmas $15.7(\mathrm{e}), 15.8(\mathrm{~d})$ has the property that $\mathcal{O}\left(A_{j}\right)=\mathcal{O}(\mathcal{T}) \cap \mathcal{F}\left(A_{j}\right)$, because $\mathcal{F}\left(A_{j}\right) \subseteq \mathcal{T}$ and $h\left(\mathcal{F}\left(A_{j}\right)\right)=h(\mathcal{T})$ following from Lemmas 15.7-15.8. Now it becomes obvious that the quotient group $\mathcal{F}\left(A_{j}\right) / \mathcal{O}\left(A_{j}\right)$ embeds into $\mathcal{T} / \mathcal{O}(\mathcal{T})$. Again referring to Lemmas 15.7-15.8, we see that our claim holds for the group $\mathcal{K}\left(A_{j}\right)$ if and only if it is true for $\mathcal{T}$ with $h(\mathcal{T})<j$. It remains to repeat the reasoning in [Iv2] to see that the direct factor $\mathbb{D}_{2}(2 n)$ (which is the second dihedral group of order $2 n$ coming from application of the induction hypothesis to $\mathcal{T})$ can be turned into $\mathbb{D}\left(2 n_{2}\right)$.

2. In proving the second (refined) version of Lemma 15.9, we consider 2 cases as in [Iv2]. But first we note that if $j=0$, then the group $\mathcal{G}$ embeds into $G(0)$ by Lemma 37 , in other words, $E=\mathcal{G}$.

Let $j>0$. By the definition, we have $\mathcal{O}(\mathcal{G}) \subseteq \mathcal{O}\left(A_{j}\right)$.

First assume $h\left(\mathcal{F}\left(A_{j}\right)\right)=0$. Then, by Lemma $37, \mathcal{F}\left(\mathcal{A}_{j}\right) \subseteq G(0)$. Hence, by Lemma 10.1(a), the group $\mathcal{K}\left(A_{j}\right)$ is an extension of $\mathcal{F}\left(\mathcal{A}_{j}\right)$ by a cyclic group $\mathbb{C}(n)$ of order $n$ or by a dihedral subgroup $\mathbb{D}(2 n)$ of order $2 n$. Hence we can put $E=\mathcal{K}\left(A_{j}\right)$. Note that then

$$
\mathcal{O}(\mathcal{G}) \subseteq \mathcal{O}\left(A_{j}\right)=\mathcal{F}\left(A_{j}\right)
$$

Now assume $h\left(\mathcal{F}\left(A_{j}\right)\right)>0$. Renaming epimorphisms $\kappa_{1}, \kappa_{2}$ of Lemmas 15.7-15.8 [Iv2] by $\Psi_{1}, \Psi_{2}$, respectively, we have from Lemmas $15.7-15.8$ that $\operatorname{ker} \Psi_{1} \cap \operatorname{ker} \Psi_{2}=$ $\{1\}$. As in [Iv2], by induction on $h(\mathcal{G})=j$, we obtain that the group $\mathcal{G}$ along with $\mathcal{K}\left(A_{j}\right)$ embeds into

$$
\mathbb{D}_{1}(2 n) \times\left(\mathbb{D}_{2}(2 n) \times \mathbb{D}\left(2 n_{2}\right)^{j-1} \times E\right),
$$

where $E$ is an elementary associated with $G(0)$ finite group. Under this embedding, denoted by $\Xi$, the projection of $\mathcal{F}\left(A_{j}\right)$ onto the first factor is trivial. Since $\mathcal{O}(\mathcal{G}) \subseteq$ $\mathcal{F}\left(A_{j}\right)$ (as above), we have by induction that $\mathcal{O}(\mathcal{G})=\mathcal{O}\left(A_{j}\right) \cap \mathcal{G} \subseteq F$, where $F \subseteq G(0)$. Any element of odd order in $\mathcal{K}\left(A_{j}\right) \cap \mathbb{D}_{2}(2 n)$ is trivial by Lemma 18.5(a) (in rank $j-1<i$ ), because $\mathcal{O}\left(A_{j}\right) \subseteq F \subseteq E$ and, again by induction, $E \cap \mathbb{D}_{2}(2 n)=\{1\}$. Consequently, as in [Iv2], the direct factor $\mathbb{D}_{2}(2 n)$ can be replaced by $\mathbb{D}\left(2 n_{2}\right)$, as required.

The statement of Lemma 15.10 [Iv2] is modified as follows. Part (a) is literally retained. Part (b) holds for elements $X, Y \in \mathcal{G}$ in the quotient group $\mathcal{G} / \mathcal{O}(\mathcal{G})$. In addition, we claim in part (b) that

$$
Y^{k_{0}} X Y^{-k_{0}} \in\left\langle Y^{\ell} X Y^{-\ell} \mid \ell=0,1, \ldots, k_{0}-1\right\rangle \subseteq G(i),
$$

where $k_{0}$ is the minimal positive integer such that $2^{k_{0}-3}$ is greater than the order of any finite subgroup of $\mathcal{G}$ ( $k_{0}$ exists by Lemma 18).

Proving this, recall that $n$ is divisible by $2^{k_{0}+5} n_{0}$, in particular, $n$ is divisible by $4 n_{0}$. Then, as in [Iv2], part (a) is immediate from Lemma 15.9. Part (b) is also obvious by Lemma 15.9 except for inclusion (32). To prove this inclusion, denote

$$
\mathcal{G}_{r}=\left\langle Y^{\ell} X Y^{-\ell} \mid \ell=0,1, \ldots, r\right\rangle \subseteq \mathcal{G}
$$


where $r \geq 0$. It follows from the proven analog of Lemma 15.10(b) [Iv2] that for every $r \geq 4$ it is true that $Y^{r} X Y^{-r} \in \mathcal{G}_{3} \mathcal{O}(\mathcal{G})$. Then for every $\ell$ with $\ell \geq 3$ we have $\mathcal{G}_{\ell} \neq \mathcal{G}_{\ell+1}$ if and only if

$$
\mathcal{G}_{\ell} \cap \mathcal{O}(\mathcal{G}) \neq \mathcal{G}_{\ell+1} \cap \mathcal{O}(\mathcal{G})
$$

Since $|\mathcal{O}(\mathcal{G})|<2^{k_{0}-3}$, the ascending chain

$$
\mathcal{G}_{3} \cap \mathcal{O}(\mathcal{G}) \subset \mathcal{G}_{4} \cap \mathcal{O}(\mathcal{G}) \subset \cdots \subset \mathcal{G}_{\ell_{0}} \cap \mathcal{O}(\mathcal{G})
$$

of subgroups of $\mathcal{O}(\mathcal{G})$ must have length $\ell_{0}-3<k_{0}-3$. Therefore the chain

$$
\mathcal{G}_{3} \subseteq \cdots \subseteq \mathcal{G}_{\ell} \subseteq \ldots
$$

stabilizes at some $\ell_{0}<k_{0}$. But then $Y^{\ell_{0}+1} X Y^{-\left(\ell_{0}+1\right)} \in \mathcal{G}_{\ell_{0}}$ and (32) becomes obvious.

To prove Lemma 16.1 it suffices to note that the cases when $h(\mathcal{G})=0$ and $h\left(\mathcal{F}\left(A_{j}\right)\right)=0$ are obvious and we can proceed as in [Iv2].

In the statement of Lemma 16.2 instead of six words $X_{1}, X_{2}, \ldots, X_{6}$ we now consider $k_{0}+2$ words $X_{1}, X_{2} \ldots, X_{k_{0}+2}$, where $k_{0}$ is the constant of Lemma 15.10(b). (Making an informal comment, we say that the constant $k_{0}$ now plays the same role as that 4 does in [Iv2]; cf. Lemma 15.10(b) [Iv2] and (32).)

Respectively, in the proof of Lemma 16.2 the number $2^{9}=2^{4+5}$ is replaced by $2^{k_{0}+5}$. In particular, $n$ is now divisible by $2^{k_{0}+5}$. We must also observe that due to Lemma 15.9 any finite subgroup $\mathcal{G}$ of $G(i)$ is isomorphic to a subgroup of the direct product of dihedral groups as in [Iv2] and the group $\mathcal{G} / \mathcal{G}^{n_{0}}$ of exponent $n_{0}$. Recall $n$ is divisible by $2^{k_{0}+5} n_{0}$. Hence, repeating the proof of Lemma 16.2 [Iv2], we have that the projections of the words $\left(Z_{1} Z_{2}\right)^{\frac{n}{4}}$ and $R$ onto the direct factor $\mathcal{G} / \mathcal{G}^{n_{0}}$ are trivial and so they do not change anything in the arguments of [Iv2].

Analogously, in Lemma 16.4 the inequality $k \geq 4$ is replaced by $k \geq k_{0}$ and the subgroup $\left\langle X_{1}, \ldots, X_{3}\right\rangle$ is now $\left\langle X_{1}, \ldots, X_{k_{0}-1}\right\rangle$. The proof is retained for $j>0$. If $j=0$ then the ascending chain of subgroups

$$
\{1\} \subset\left\langle X_{1}\right\rangle \subset\left\langle X_{1}, X_{2}\right\rangle \subset \cdots \subset\left\langle X_{1}, \ldots, X_{\ell_{0}}\right\rangle
$$

cannot have more than $k_{3}-3$ strict inclusions by choice of $k_{0}$ and Lemma 37 . Hence $\ell_{0} \leq k_{0}-3$. But any equality $\left\langle X_{1}, \ldots, X_{\ell-1}\right\rangle=\left\langle X_{1}, \ldots, X_{\ell}\right\rangle$ for $\ell \leq k_{0}$ obviously implies

$$
\left\langle X_{1}, \ldots, X_{k_{0}-1}\right\rangle=\left\langle X_{1}, \ldots, X_{k_{0}}\right\rangle
$$

which means that Lemma 16.4 is proven.

In Lemma 16.5 instead of $X_{0}, X_{1}, \ldots, X_{6}$ we put $X_{0}, X_{1}, \ldots, X_{k_{0}+2}$. Similarly, instead of $X_{0}, X_{1}, \ldots, X_{7}$ we put $X_{0}, X_{1}, \ldots, X_{k_{0}+3}$ in Lemma 16.6. The proofs are retained when $j>0$. If $j=0$ (it is possible only in proving Lemma 16.6), then it follows from consideration of a chain of finite subgroups of $G(0)$ and choice of $k_{0}$ that the word $W$ still normalizes the subgroup $\left\langle X_{0}, X_{1}, \ldots, X_{k_{0}-1}\right\rangle$. 


\section{Solvable Pairs of Vertices}

Lemma 41. Let $\Delta$ be a reduced disk diagram of rank $i$ with $\partial \Delta=p_{1} q_{1} p_{2} q_{2}$, $\left\|q_{1}\right\| \geq \Omega, \phi\left(q_{1}\right)$ and $\phi\left(q_{2}\right)$ be $A_{i_{1}}$ - and $A_{i_{2}}^{\varepsilon_{2}}$-periodic words, $\varepsilon_{2}= \pm 1$, respectively, where $A_{i_{1}}, A_{i_{2}}$ are small periods, and $\Delta$ has no cells that are compatible with $q_{1}$ and $q_{2}$. In addition, assume that either $\Delta$ itself is a contiguity subdiagram between $q_{1}$ and $q_{2}$ or $\max \left(\left\|p_{1}\right\|,\left\|p_{2}\right\|\right) \leq C$. Then $i_{1}=i_{2}$ and there are phase vertices $o_{1} \in q_{1}$, $o_{2} \in q_{2}$ and a path $t=o_{1}-o_{2}$ in $\Delta$ such that $|t|<\delta_{1}\left|A_{i_{1}}\right|$ and $\phi(t) \in F\left(A_{i_{1}}\right) \subseteq G(0)$ provided $\varepsilon_{2}=-1$ and $\phi(t) \in E^{-}\left(A_{i_{1}}\right) \subseteq G(0)$ provided $\varepsilon_{2}=1$.

Proof. First let us show that $r(\Delta)=0$. Suppose $\Delta$ is a contiguity subdiagram between $q_{1}$ and $q_{2}$. Then it follows from Lemma 9.1(c) that $r(\Delta)=0$, because no cell can have a contiguity subdiagram of degree $\geq \beta$ to a smooth section with a small period.

Suppose $\max \left(\left\|p_{1}\right\|,\left\|p_{2}\right\|\right) \leq C$. This case is reduced to the above as follows. Applying Lemma 6.5 in standard way, we can find two diagrams, say $\mathrm{E}_{j}, j=1,2$, in $\Delta$ in such a way that $\partial \mathrm{E}_{j}=s_{1}^{j} t_{1}^{j} s_{2}^{j} t_{2}^{j}$ and

$$
\left|s_{1}^{j}\right|+\left|t_{1}^{j}\right|+\left|s_{2}^{j}\right|+\left|t_{2}^{j}\right|<(C+\Lambda)(1+\mu) .
$$

By Lemmas 6.2 and 26, $r\left(\mathrm{E}_{j}\right)=0$ for either $j$. Therefore, $r(\Delta)=0$ as well.

Reasoning as in the proof of Lemma 43 [Ol5], we obtain an equality

$$
A_{i_{1}}^{r} \stackrel{0}{=} X A_{i_{2}}^{\ell} X^{-1}
$$

where $r>0$ and $X \equiv \phi(x)$ with a simple path $x$ that connects some phase vertices of $q_{1}$ and $q_{2}$ and $|x|<2 C+4 \lambda$. In addition, the lengths \|\| of the paths in which $x_{-} \in q_{1}, x_{+} \in q_{2}$ decompose $q_{1}, q_{2}$, respectively, are sufficiently large, say $>\Omega / 4$.

It follows from Lemma 7 and the definition of a small $p$ period that $i_{1}=i_{2}$. By Lemma 8, $r= \pm \ell$ and, by Lemma $7, X \in E\left(A_{i_{1}}\right)$. Consider two cases:

1. $r=\ell$, that is, $X \in E^{+}\left(A_{i_{1}}\right)$ and $\varepsilon_{2}=-1$ (so both $\phi\left(q_{1}\right)$ and $\phi\left(q_{2}\right)^{-1}$ are $A_{i_{1}}$-periodic words as in Lemma $\left.43[\mathrm{Ol}]\right)$. Then we have $X \stackrel{0}{=} A_{i_{1}}^{d} T$, where $T \in F\left(A_{i_{1}}\right)$. Inasmuch as $|T|<\delta_{0}$ by Lemma 23 and $|X|<2 C+4 \lambda$, we get $\left|A_{i_{1}}^{d}\right|<2 C+4 \lambda+\delta_{0}$. Hence, by Lemmas $11-12$,

$$
\left\|A_{i_{1}}^{d}\right\| \leq 3 \kappa_{1}^{-1}\left(2 C+4 \lambda+\delta_{0}+\kappa_{2}\right)<\Omega / 4
$$

Therefore, changing the phase vertex of $q_{1}$, we can replace the word $X \stackrel{0}{=} A_{i_{1}}^{d} T$ by $X^{\prime} \stackrel{0}{=} T$. This means that the lemma is proven.

2. $r=-\ell$, that is, $X \in E^{-}\left(A_{i_{1}}\right)$ and $\varepsilon_{2}=1$ (so both $\phi\left(q_{1}\right)$ and $\phi\left(q_{2}\right)$ are $A_{i_{1}}$-periodic words). Then $E^{+}\left(A_{i_{1}}\right)$ is of index 2 in $E\left(A_{i_{1}}\right)$. Hence the quotient $E\left(A_{i_{1}}\right) / F\left(A_{i_{1}}\right)$ is an infinite dihedral group and $X$ is an $F\left(A_{i_{1}}\right)$-involution. Thus we have a required path $t=x$ (no need to change phase vertices if we put $\delta_{1}>$ $2 C+4 \lambda$.)

The constants $K_{1}=214, \ldots, N=484$ in the list (17.1) on p. 212 [Iv2] are now supplied with the superscript $\Omega$ and replaced by $K_{1}^{\Omega}=214 \Omega, \ldots, N^{\Omega}=484 \Omega$.

In the statement of Lemma 17.1 it is additionally required that $|A| \geq C$ and the inequality $|A| \leq\left|A_{i}\right|+1$ is replaced by the condition that no word of length $<|A|$ 
is simple in rank $i$. Instead of $|p|$ and $|q|$ we put $\|p\|$ and $\|q\|$. The conclusion of Lemma 17.1 is now that $h\left(\phi\left(d_{1}\right)\right)=0$ in $G(i)$. (These changes, however, do not affect estimates in the proof because, by Lemma 13, taking, for instance, $\theta_{0}=10^{-5}$, one has $|p| \geq\left(1-10^{-5}\right)\|p\|$ by virtue of choice of $C>C\left(10^{-5}, G(0)\right)$.)

In the proof of Lemma 17.1, applying Lemma 31 (instead of Lemma 6.1), we must add additional term $2 L$ to the right part of (17.3). The same correction is made in (17.6), but inequality (17.7) is retained for $|A| \geq C \gg L$.

Next, the inequality $\left|d_{1}\right|<|A|$ implies, in view of Lemmas $24-25$, the finiteness of the order of $\phi\left(d_{1}\right)$ in rank $i$ because, by the hypothesis of Lemma 17.1, $\phi\left(d_{1}\right)$ is not simple in rank $i$.

Inequality (17.8) is retained by Lemma 25 provided $A_{j}$ is a large period. Otherwise, $\left|A_{j}\right|<C \leq|A|$.

In the right parts of inequalities (17.9) and (17.11) we add $2 L$. That does not affect inequalities (17.13) and (17.14) as $|A| \geq C$.

Making $x_{2}$ and $w_{2}$ in the diagram $\Gamma_{2}$ a little shorter by applying Lemma 9.4, we pass first to the diagram $\Gamma_{3}$ with the contour $\partial \Gamma_{3}=\bar{f}_{1} x_{3} \bar{f}_{2} w_{2}$. This makes it possible to apply Lemma 9.5 to estimate $\left|f_{1}\right|+\left|f_{2}\right|$. After doing this, the proof ends as in [Iv2] provided $A_{j}$ is a large period. If $A_{j}$ is small, then we will use a modification of Lemma 17.4 that is discussed below in Sect. 14 .

We do not have analog of Lemma 17.2, replacing it with some other lemmas.

In the hypothesis of Lemma 17.3, instead of the inequality $|A| \leq\left|A_{i}\right|+1$ we require that the word $A$ is a cyclic permutation of a small period provided $|A|<C$ and that all words of length $<|A|$ are not simple in rank $i$. In the conclusion, the line $\ell=0,1,2,3$ is replaced by $\ell=0,1, \ldots, k_{0}-1$.

If $A$ is a small period, then we pick a path $t$ in accordance with Lemma 41 and note that $\phi(t) \in F(A) \subseteq G(0)$, since the words $\phi(p), \phi(q)^{-1}$ are $A$-periodic. Then all the words $A^{-\ell} T A^{\ell}$ belong to $F(A)$ for $A$ normalizes $F(A)$. The normality of the subgroup $\left\langle A^{-\ell} T A^{\ell} \mid \ell=0,1, \ldots, k_{0}-1\right\rangle$ of $\langle A, T\rangle$ is now explained as in Lemmas 16.4 and 16.5 .

Now consider the case $|A| \geq C$. As in proving Lemma 17.1, we see that the word $\phi\left(d_{1}\right)$ is of finite order in rank $i$. We introduce the period $A_{m}$ only when $h\left(\phi\left(d_{1}\right)\right)>0$.

As above (see Sects. 10-11), the coefficient 7 in inequality (17.31) is replaced by $7 \delta_{1} \kappa_{1}^{-1}$.

In the definition of the divergence div, we use the length \|\| .

In the definition of a solvable pair of vertices, we make the following changes. First, if $j>0$, then we require that $\operatorname{div}\left(o_{p}, o_{q}\right)<0.6|\partial \Pi|$, where $\Pi$ is a cell of rank $j$. Second, if $j=0$, then we lift the restriction "either $o_{p}=\left(x_{t}\right)_{+}=\left(p_{t}\right)_{-}$, $o_{q}=\left(x_{t}\right)_{-}=\left(q_{t}\right)_{+}$, or $o_{p}=\left(y_{t}\right)_{-}=\left(p_{t}\right)_{+}, o_{q}=\left(y_{t}\right)_{+}=\left(q_{t}\right)_{-}$" and require that $\left|o_{p}-o_{q}\right|<11 L$ for vertices $o_{p}, o_{q}$ with $\operatorname{div}\left(o_{p}, o_{q}\right)=0$.

The following Lemma 42 is an analog of Lemma 17.3.1. We will prove it here in a simpler way (and without making use of Lemma 17.2 whose analog is missing in this article).

Lemma 42. The diagram $\Delta^{\prime \prime}\left(m_{1}, m_{2}\right)$ contains a solvable pair of vertices. In addition, if $r(\Delta)=0$ then any phase vertex of $p$ can be taken as a vertex of a solvable pair of vertices in $\Delta$.

Proof. First assume $j>0$ and consider a cell $\Pi$ of rank $j$ which, by Lemma 9.1(c), 


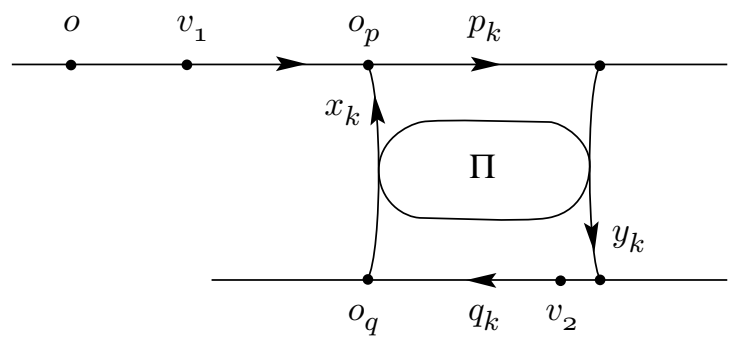

FiguRE 6

is the principal cell of a bond $\mathrm{E}_{k}$ with the standard contour $\partial \mathrm{E}_{k}=x_{k} p_{k} y_{k} q_{k}$, where $\left|x_{k}\right|,\left|y_{k}\right|<\chi^{\prime}|\partial \Pi|$. Put $o_{p}=\left(x_{k}\right)_{+}$and $o_{q}=\left(x_{k}\right)_{-}$, see Figure 6. By $o$ denote a vertex of the path $p_{1} r_{1} \ldots p_{k-1} r_{k-1}$ (or of the path $s_{k-1} q_{k-1} \ldots s_{1} q_{1}$, see Figure 9.2 [Iv2] $)$ such that $\operatorname{div}\left(o, o_{q}\right)=0\left(\operatorname{div}\left(o_{p}, o\right)=0\right.$, respectively $)$.

The lemma will be proven if we disprove the inequality $\|z\| \geq 0.6|\partial \Pi|$ for the path $z=o-o_{p}$ going along $p$.

If after gluing the diagram $\Delta^{\prime \prime}\left(m_{1}, m_{2}\right)$ into an annular diagram (denoted in [Iv2] by $\left.\Delta_{0}^{\prime \prime}\left(m_{1}, m_{2}\right)\right)$, we encounter a reducible cell of some rank $j_{0}>0$, then the word $\phi\left(o-o_{q}\right)$ is conjugate in rank $j$ to an $\mathcal{F}\left(A_{j_{0}}\right)$-involution, hence, by Lemma 10.1 (for more details see the proof of inequality (10.16) in [Iv2]), to a word of length less than

$$
\left(3 \delta_{1}+1\right)\left|A_{j_{0}}\right|<\left(1-\alpha^{\prime}\right)^{-1}\left(3 \delta_{1}+1\right) \kappa_{1}^{-1} n^{-1}|\partial \Pi|
$$

by Lemma 26 for $\left\|A_{j_{0}}\right\| \leq\left\|A_{j}\right\|$. If $h\left(\phi\left(d_{1}\right)\right)=h\left(\phi\left(o-o_{q}\right)\right)=0$ in rank $i$, then $\phi\left(o-o_{q}\right)$ is conjugate in rank $j$ to a word of length $<C_{0}$. In any case, the word $\phi\left(z x_{k}^{-1}\right)$ is conjugate in rank $j$ to a word of length $<\gamma|\partial \Pi|$.

Since

$$
|A|>(2.22)^{-1}|\partial \Pi|>\frac{1}{3} \kappa_{1} n
$$

by Lemmas 9.5 and 12, Lemma 13 and LPP enable us to assume that $\left|p_{0}\right| \geq$ $\left(1-\theta_{0}\right)\left\|p_{0}\right\|$ for every subpath $p_{0}$ of $p$ (or $q$ ).

Now let $Z_{1} Z_{2} \equiv Z \equiv \phi(z)$ be a decomposition of the word $Z \equiv \phi(z)$ into its left and right parts:

$$
0 \leq\left\|Z_{2}\right\|-\left\|Z_{1}\right\| \leq 1
$$

Then the word $Z_{2} X_{k}^{-1} Z_{1}$, where $X_{k} \stackrel{i}{=} \phi\left(x_{k}\right)$ is reduced in rank $i$, is equal in rank $i$ by Lemma $32(\mathrm{~b})$ to a word $W$ with

$$
|W|<4\left|X_{k}\right|+5 \gamma|\partial \Pi|+6 L+6 \theta_{0}|Z| .
$$

Hence, it follows from $\left|X_{k}\right|<\left(\chi^{\prime}+2 \gamma\right)|\partial \Pi|$ that

$$
|W|<5 \chi^{\prime}|\partial \Pi|+6 \theta_{0}|Z|
$$

Let us pick a vertex $v_{1} \in z$ that defines the decomposition $z=z_{1} z_{2}$, where $\phi\left(z_{1}\right) \equiv Z_{1}$ and $\phi\left(z_{2}\right) \equiv Z_{2}$ (Figure 6). Since $\operatorname{div}\left(o, o_{q}\right)=0$, there is a vertex $v_{2}$ on $q^{-1}$ (or on an $A$-periodic continuation of $q^{-1}$ ) such that $\phi\left(o_{q}-v_{2}\right) \equiv Z_{1}$, where $o_{q}-v_{2}$ is a subpath of $q^{ \pm 1}$. 


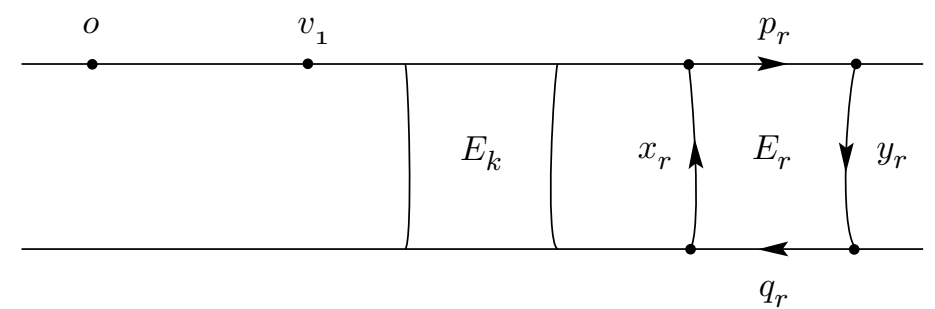

FigURE 7

1. Keeping the notation of [Iv3], let $v_{2} \in q_{k}$ and denote $q_{k}=q_{k 1} q_{k 2}$, where $\left(q_{k 1}\right)_{+}=v_{2}$. Then $\left|q_{k 2}\right| \geq \frac{1}{2}|Z|>0.3|\partial \Pi|$. Hence it follows from Lemmas 3.4, 3.1, 13,27 , and 6.1 that

$$
\left|q_{k 1}\right| \leq\left\|q_{k}\right\|-\left|q_{k 2}\right| \leq\left(1-\theta_{0}\right)^{-1} \rho^{-1}(\alpha+2 \gamma)|\partial \Pi|-0.3|\partial \Pi| .
$$

It follows from this inequality and (33) that

$$
\left|W \phi\left(q_{k 1}\right)^{-1} \phi\left(y_{k}\right)^{-1}\right|<\left(\left(1-\theta_{0}\right)^{-1} \rho^{-1}(\alpha+2 \gamma)+5 \chi^{\prime}-0.3\right)|\partial \Pi|+6 \theta_{0}|Z| .
$$

On the other hand, the word $W \phi\left(q_{k 1}\right)^{-1} \phi\left(y_{k}\right)^{-1}$ is equal in rank $i$ to the word $Z_{2} \phi\left(p_{k}\right)$ of length

$$
\left|Z_{2} \phi\left(p_{k}\right)\right|>\left(1-\theta_{0}\right)\left(\left(\rho\left(\chi-\alpha-4 \alpha^{\prime}\right)-2 \gamma\right)|\partial \Pi|+\frac{1}{2}|Z|\right),
$$

following from Lemmas 3.4, 3.1, 13, 27, and 6.1.

Thus a contradiction to Lemma 6.1 is obtained because the word $Z_{2} \phi\left(p_{k}\right)$ is $A$-periodic, however,

$$
\begin{aligned}
\rho\left(1-\theta_{0}\right)\left(\rho\left(\chi-\alpha-4 \alpha^{\prime}\right)-4 \gamma\right) & >\left(1-\theta_{0}\right)^{-1} \rho^{-1}(\alpha+2 \gamma)+5 \chi^{\prime}-0.3 \quad(\mathrm{LPP}) \\
\frac{\rho}{2}\left(1-\theta_{0}\right) & >6 \theta_{0} \quad(\mathrm{LPP}) .
\end{aligned}
$$

2. Now assume that $v_{2} \in s_{\ell_{1}}$ for some $\ell_{1} \geq k$. Then, by the maximality of $j=r(\Pi)$, Lemmas 12 and 9.1 yield that

$$
\left|\partial \Delta_{\ell_{1}}\right| \leq 240 \beta^{-1} \kappa_{1}^{-1} n^{-1}|\partial \Pi| .
$$

In particular, $v_{2}$ can be connected along $\partial \Delta_{\ell_{1}}$ with the vertex $\left(y_{\ell_{1}}\right)_{-} \in p_{\ell_{1}}$ by a very short (relative to $|\partial \Pi|)$ path $u$. Consequently, comparing $|W \phi(u)|$ and $\left|\phi\left(v_{1}-\left(y_{\ell_{1}}\right)_{-}\right)\right|$, where $v_{1}-\left(y_{\ell_{1}}\right)_{-}$of a subpath of $p^{\prime \prime}\left(m_{1}, m_{2}\right)$, we get a contradiction as above.

3. Let $v_{2} \in q_{\ell_{1}}$ for $\ell_{1}>k$. Consider a path $t$ of the form $t=\left(v_{2}-\left(y_{\ell_{1}}\right)_{+}\right) y_{\ell_{1}}^{-1}$ (see Figure 7 ), where $\left(v_{2}-\left(y_{\ell_{1}}\right)_{+}\right)$is a subpath of $q_{\ell_{1}}^{-1}$. Recall $\pi_{\ell_{1}}$ denote the principal cell (if any) of the bond $\mathrm{E}_{\ell_{1}}$. By Lemmas 3.4, 3.1, 13, 27, and 6.1, we have

$$
|t|-\rho\left|p_{\ell_{1}}\right|<\left(\rho^{-1}(\alpha+4 \gamma)+\chi^{\prime}+4 \gamma-\rho\left(\rho\left(\chi-\alpha-4 \alpha^{\prime}\right)+4 \gamma\right)\right)\left|\partial \pi_{\ell_{1}}\right| .
$$

The right part of this inequality is less than $\mu^{\prime} \kappa_{1}^{-1}|\partial \Pi|$ following from choice of $\Pi$, Lemma 26, and LPP. 
If $\mathrm{E}_{\ell_{1}}$ is a 0 -bond, then the estimate for this difference will only improve.

Note that $z_{2}, p_{\ell_{1}}$ are disjoint subpaths of $p^{\prime \prime}\left(m_{1}, m_{2}\right)$ with

$$
\operatorname{dis}_{p^{\prime \prime}\left(m_{1}, m_{2}\right)}\left(\left(z_{2}\right)_{+},\left(p_{\ell_{1}}\right)_{-}\right) \geq 0 .
$$

Hence, by Lemma 14,

$$
\operatorname{dis}_{p^{\prime \prime}\left(m_{1}, m_{2}\right)}\left(\left(z_{2}\right)_{-},\left(p_{\ell_{1}}\right)_{+}\right) \geq\left|z_{2}\right|+\left|p_{\ell_{1}}\right|-2 \lambda_{0} .
$$

On the other hand, by Lemma 6.1, we have

$$
\rho \operatorname{dis}_{p^{\prime \prime}\left(m_{1}, m_{2}\right)}\left(\left(z_{2}\right)_{-},\left(p_{\ell_{1}}\right)_{+}\right) \leq|W|+|t| .
$$

Consequently, to obtain a contradiction, it suffices to establish that

$$
\rho\left(\left|z_{2}\right|+\left|p_{\ell_{1}}\right|\right)>|W|+|t|+2 \lambda_{0} .
$$

But this inequality follows from the following two estimates:

$$
\begin{aligned}
|t|-\rho\left|p_{\ell_{1}}\right|<\mu^{\prime} \kappa_{1}^{-1}|\partial \Pi|, \\
\rho\left|Z_{2}\right|-|W|>\left(\rho-6\left(1-\theta_{0}\right)^{-1} \theta_{0}\right)\left|Z_{2}\right|-5 \chi^{\prime}|\partial \Pi| \\
\geq\left(0.3 \cdot \frac{1}{2}\left(1-\theta_{0}\right)\left(\rho-6\left(1-\theta_{0}\right)^{-1} \theta_{0}\right)-5 \chi^{\prime}\right)|\partial \Pi| \\
>2 \lambda_{0}+\mu^{\prime} \kappa_{1}^{-1}|\partial \Pi| \quad(\mathrm{LPP}),
\end{aligned}
$$

the first of which was obtained above and the second one follows from (33), since $\left|Z_{2}\right| \geq\left(1-\theta_{0}\right)|| Z_{2} \| \geq \frac{1}{2}\left(1-\theta_{0}\right)|Z|$ by Lemma 13 .

It remains to consider the case $j=0$, that is, the entire diagram $\Delta^{\prime \prime}\left(m_{1}, m_{2}\right)$ is a 0 -bond. Then it follows from Lemmas 11-12 that any vertex $o_{p} \in p^{\prime \prime}\left(m_{1}, m_{2}\right)$ can be joined with a vertex $o_{q} \in q^{\prime \prime}\left(m_{1}, m_{2}\right)$ by a path of length $<2 \Lambda$.

In the notation introduced above (but now we put $X$ instead of $X_{k}$ ), we have, as in the case $j>0$ above, that

$$
\left|Z_{2} X^{-1} Z_{1}\right|<8 \Lambda+10 L+4 C_{0}+3 \theta_{0}|Z| .
$$

By Lemmas 11-12, the vertex $v_{2}$ can be joined with a vertex on $p$ (but not on $z$ ) by a path of length $<2 \Lambda$. As above, comparison of lengths of these two paths yields the inequality

$$
|z|-\lambda_{0}<8 \Lambda+10 L+4 C_{0}+3 \theta_{0}|Z|+2 \Lambda .
$$

Taking into account the order of choosing constants, we can suppose that

$$
|Z|+|X|<|Z|+2 \Lambda<11 L,
$$

as required.

Remark. The coefficient 0.6 of $|\partial \Pi|$ in the above argument involved in proving Lemma 42 can be replaced by 0.01 etc. As a matter of fact, the trick used to prove the existence of solvable pairs of vertices in $\Delta^{\prime \prime}\left(m_{1}, m_{2}\right)$ makes it possible to 
simplify some proofs in [Iv2] (besides, the lengths | | and \|| || are identical in [Iv2] and the coefficients $\kappa_{1}, \lambda_{0}$ are not needed in [Iv2]). In addition, there is no longer need in Lemma 17.2, in double definitions of compatibility (ordinary and weak), double properties of being smooth and being reduced (ordinarily and strictly); it suffices to define only weak compatibility and properties of being strictly smooth and being strictly reduced.

\section{The Inductive Step from the Group $G(i)$ to $G(i+1)$}

In Lemmas 17.3.2 (as well as in Lemma 17.3.1), we, as always, replace $n\left|A_{j}\right|$ by $\left|A_{j}^{n}\right|$. In Cases (Z1)-(Z2), we use the length \|\| . Analogously, in part (c) of the conclusion of Lemma 17.3.3 we say that $\left\|q\left(m_{3}, m_{4}\right)\right\|>\left(L^{\Omega} / 2-2.2\right)|A|$ (note the $L$ in (17.1) [Iv2] differs from the $L$ of this article, see (12)). The lengths \|\| are estimated in inequality (17.64) as well.

The diagrams $\Delta(\ell)$ are now constructed for all $\ell$ from 1 to $k_{0}+3$. The coefficients 7.4 and 8.1 in the statement of Lemma 17.3 .4 are replaced by $k_{0}+4, k_{0}+5$, respectively, and the coefficients 9.7 and 10.3 in the proof of Lemma 17.3 are replaced by $k_{0}+6, k_{0}+7$, respectively.

After removal of the cell $\Pi^{0}$ from $\Delta_{k^{0}+1}^{0}$ in the proof of Lemma 17.3.5, we now need to replace the contour $c=\partial \Pi^{0}$ (left after the removal) with $\|c\|=\left\|\partial \Pi^{0}\right\|$ by a contour $c^{\prime}$ with $\left\|c^{\prime}\right\|=\left|\partial \Pi^{0}\right|$ by pasting a disk diagram of rank 0. By Lemmas 12 and 21 , estimate (17.69) for $\left|z_{1}^{0}\right|$ can get worse by no more than $L+\left|A_{j}\right|$. This will not affect subsequent inequalities, because $A_{j}$ is a large period.

In the statement of Lemma 17.3.6 the coefficient 16.6 is replaced by $k_{0}+13$. In the end of its proof, we observe that $\left(\rho^{-1}-1\right) \ell$ is sufficiently small, since $\ell \leq k_{0}+3$ and the parameter $\rho$ is chosen after $k_{0}$ (LPP).

In the end of the proof of Lemma 17.3, we need to consider the case when $j=r\left(\Delta^{\prime \prime}\left(m_{1}, m_{2}\right)\right)=0$. Let $o_{1}, o_{2}, \ldots$ be distinct phase vertices on the section $p^{\prime \prime}\left(m_{1}, m_{2}\right)$ the number of which is more than $400 \Omega$. By Lemma 42 each of them can be connected with a phase vertex $o_{1}^{\prime}, o_{2}^{\prime}, \ldots$, respectively, on the section $q^{\prime \prime}\left(m_{2}, m_{1}\right)$ by a simple path $t_{1}, t_{2}, \ldots$ of length $<11 L$. Since $\Omega$ is chosen after $L$, we can conclude as in Lemma $43[\mathrm{Ol}]$ (or Lemma 41 of Sect. 13) that for some $t_{\ell^{\prime}}$ the label $\phi\left(t_{\ell^{\prime}}\right)$ belongs to $F(A) \subseteq G(0)$. After this we proceed as in the case of a small period in proving Lemma 42.

In the statement of Lemma 17.4, part (3) is weakened: if $A_{m}$ is a small period, then we require only that $\|p\|>M_{1}^{\Omega}|A|$.

In the proof, the case of a large period is handled as in [Iv2]. If $A_{m}$ is a small period, then $r\left(\Delta\left(m_{1}, m_{2}\right)\right)=0$ for the same reasons as in Lemma 41. But in this situation, Lemma 41 applies to the constructed diagram $\Delta_{0}$ and enables us to finish the proof of Lemma 17.4 as in [Iv2].

In the hypothesis of Lemma 18.1, $\Delta$ can also be an annular reduced diagram of rank $i$ with contours $p$ and $q$. Next, the assumption that $\phi(\Delta)$ is cyclically reduced is replaced by that $\partial \Delta=p q$ (if $\Delta$ is annular then $p, q$ are the contours of $\Delta$ ), where $p$ is 0 -geodesic and $q$ is either smooth or 0 -geodesic. In the conclusion of Lemma $18.1, u^{-1}$ is not necessarily a subpath of $\partial \Delta: u$ is just the contiguity arc $\Gamma \wedge \pi$, where $\Gamma$ is a contiguity subdiagram of rank 0 between $\pi$ and one of $p, q$ with $\|u\| \geq \beta\|\partial \pi\|$.

It is possible to prove the existence of the period of any rank, but we will not use this and state in Lemma 18.2 that the period $A_{i+1}$ (if any) is a simple word in 
rank $i$. This now follows from the definition of a period and Lemma 24 .

In Lemma $18.3, \ell$ changes from 0 to $k_{0}-1$.

The words $T_{k, \ell} \equiv A_{i+1}^{\ell} \phi\left(r^{k}\right) A_{i+1}^{-\ell}$ possess, by Lemma 17.3 , the properties that they have finite order in rank $i$ and

$$
T_{k, \ell} A_{i+1}^{n_{k, \ell}} T_{k, \ell}^{-1} \stackrel{i}{=} A_{i+1}^{n_{k, \ell}}
$$

for some $n_{k, \ell}>0$. It is easy to derive from these properties that all $T_{k, \ell}$ belong to a finite subgroup of $G(i)$ (a simple explanation to this is given in [Ol3]). It is also possible just to follow the proof of Lemma 18.3 with standard little changes. But the simplest way is to refer to Lemma 21.1 [Iv2] (whose proof in rank $i$ uses only those Lemmas that precede Lemma 18.3), which by Lemma 1 implies the hyperbolicity of the group $G(i)$. Then the finiteness of the subgroup generated by all $T_{k, \ell}$ simply follows from Lemma 7 applied to $G(i)$. Lemma 18.4(a) can have a similar proof.

In part (b) of Lemma 18.4 (and in subsequent Lemmas) we remove the claim on occurrences of letters. The inequality $|S|<\delta_{1}\left|A_{i+1}\right|$ is obtained as in [Iv2] provided $A_{i+1}$ is a large period. Otherwise, it is possible to cut a "long" subdiagram out of the diagram for equality (18.42). This subdiagram, as in Lemma 41, is of rank 0 . After this we will obtain an equality of the form (18.42) but now in rank 0. It remains to refer to Lemma 23, keeping in mind that $\delta_{1}>\delta_{0}$.

Before stating Lemma 18.5, define

$$
\mathcal{O}\left(A_{i+1}\right)=\mathcal{O}\left(\mathcal{F}\left(A_{i+1}\right)\right)
$$

where the right part is already correctly defined and is a normal subgroup in $\left\langle\mathcal{F}\left(A_{i+1}\right), A_{i+1}\right\rangle$ by parts (a), (c) of Lemma 40.

In the statement of Lemma 18.5(a), it is now asserted that the quotient group

$$
\mathcal{F}\left(A_{i+1}\right) / \mathcal{O}\left(\mathcal{F}\left(A_{i+1}\right)\right)=\mathcal{F}\left(A_{i+1}\right) / \mathcal{O}\left(A_{i+1}\right)
$$

is a 2 -group.

In the proof of part (a) of Lemma $18.5 \ell$ changes from 0 to $k_{0}-1$.

The proof of part (c) is retained if $h\left(\mathcal{F}\left(A_{i+1}\right)\right)>0$. Otherwise, we refer to Lemmas 37, 19 and use the divisibility of $n$ by $2 n_{0}$.

In view of Lemma 41, it now suffices to prove Lemma 19.1 only for the large period $A_{i+1}$.

In Lemma 19.2, it is now asserted that the order of the image of an $\mathcal{F}\left(A_{i+1}\right)$ involution $J$ in the quotient group $\left\langle\mathcal{F}\left(A_{i+1}\right), J, A_{i+1}\right\rangle / \mathcal{O}\left(A_{i+1}\right)$ is a positive power of 2 (note $J$ normalizes the subgroup $\mathcal{O}\left(A_{i+1}\right)$ following from Lemma 40(c)).

The proof is retained provided $h\left(\mathcal{F}\left(A_{i+1}\right)\right)>0$. Otherwise, equation (19.24) follows from Lemmas 37, 19, and the divisibility of $n$ by $2 n_{0}$.

In Lemma 19.5, we claim the existence of the words $T_{J}, T_{I}, S_{A_{i+1}}$ only in the case when $h\left(\mathcal{F}\left(A_{i+1}\right)\right)>0$.

In Lemma 20.1, we put the lengths $\|p\|,\|q\|$ and the coefficient $2^{28}$ is replaced by $\beta^{-2}$ (then by Lemma $12,|q|>\frac{1}{3} \kappa_{1} \beta^{-2}\left|A_{j}\right|$ ). Analogous corrections are made in inequalities of the proof.

In Lemmas 20.2, 20.3, the coefficients $2^{28}, 2^{29}$ are replaced by $\beta^{-2}, 4 \kappa_{1}^{-1} \beta^{-2}$, respectively. (The coefficient $\kappa_{1}$ appears in a standard way, when lengths are compared by Lemma 12.) 


\section{The Proof of Theorem A}

The constructed group

$$
G(\infty)=\left\langle G \| A_{1}^{n}=1, A_{2}^{n}=1, \ldots\right\rangle
$$

obtained from $G=G(0)$ by imposing on $G$ of relations of all ranks $i=1,2, \ldots$ satisfies the identity $x^{n} \equiv 1$ following from the definition of periods $A_{i}, i=1,2, \ldots$, Lemmas 24, 25, and 10.4.

(a) By Lemma 15 and LPP, the set of $(\Lambda, t)$-aperiodic words is infinite for $t=[\beta n]$ ([ ] is the integer part function). Therefore, to prove the infiniteness of the group $G(\infty)$ it suffices to establish that the restriction of the natural epimorphism $G \rightarrow G(\infty)$ on the set, consisting of distinct in $G(\Lambda, t)$-aperiodic words, is injective. Arguing on the contrary, we easily obtain a reduced disk diagram $\Delta$ with the contour $\partial \Delta=p q$, where $\phi(p), \phi\left(q^{-1}\right)$ are distinct in $G(\Lambda, t)$-aperiodic words. However, by the definition of a contiguity subdiagram and Lemma 18.1, one of $\phi(p), \phi\left(q^{-1}\right)$ must contain a $(\Lambda, t)$-power, contrary to the definition of $(\Lambda, t)$-aperiodicity.

(b)-(c) These parts are proved exactly as in [Iv2] on the base of Lemmas 6.2, 6.4, 21.4, and 15.9.

(d) Note that the choice of the exponent $n=n(G)$ is such that all multiples of $n$ also satisfy all of the conditions imposed on $n$. Hence, if $g \neq 1$ in $G$ and $0.3 \kappa_{1}^{-1}(k n)>|g|$, then $g \neq 1$ in any $G(i)=G(i, k n)$ by Lemmas 12 and 6.2 (because the strict rank of a reduced diagram of rank $i$ for the hypothetical equality $g \stackrel{i}{=} 1$ will be 0$)$. Consequently, $g \notin G^{k n}$ and so

$$
\bigcap_{k=1}^{\infty} G^{k n}=\{1\}
$$

Analogously, the inequality $0.3 \kappa_{1}^{-1} n>C_{0}$ and Lemma 16 mean that $G^{n}$ is a torsion free subgroup of $G$.

Theorem A is proven.

\section{REFERENCES}

[Ad] S.I. Adian, The Burnside problem and identities in groups, Nauka, Moscow, 1975; English translation: Springer-Verlag, 1979. MR 80d:20035

[Bm] G. Baumslag, Topics in combinatorial group theory, Birkhäuser, 1993. MR 94j:20034

[Br] W. Burnside, On unsettled question in the theory of discontinuous groups, Quart. J. Pure and Appl. Math. 33 (1902), 230-238.

[CDP] E. Coornaert, T. Delzant, and A. Papadopoulos (eds.), Géométrie et théorie des groupes: Les groupes, hyperboliques de Gromov, Lecture Notes in Math., vol. 1441, Springer-Verlag, 1991. MR 92f:57003

[GH] E. Ghys and P. de la Harpe (eds.), Sur les groupes hyperboliques d'aprés Mikhael Gromov, Birkhäuser, 1990. MR 92f:53050

[GS] S.M. Gersten and H. Short, Small cancellation theory and automatic groups, Invent. Math. 102 (1990), 305-334. MR 92c:20058

[Gp] N. Gupta, On groups in which every element has finite order, Amer. Monthly 96 (1989), 297-308. MR 90d:20073

[Gr] M. Gromov, Hyperbolic groups, in Essays in Group Theory (S. M. Gersten, ed.), M.S.R.I. Pub. 8, Springer, 1987. MR 88e:20004

[IO] S.V. Ivanov and A.Yu. Ol'shanskii, Some applications of graded diagrams in combinatorial group theory, London Math. Soc. Lecture Notes Ser., vol. 160 (1991), Cambridge Univ. Press, Cambridge and New York, 1991, pp. 258-308. MR 92j:20022 
[Iv1] S.V. Ivanov, On the Burnside problem on periodic groups, Bulletin of the AMS 27 (1992), no. 2, 257-260. MR 93a:20061

[Iv2] S.V. Ivanov, The free Burnside groups of sufficiently large exponents, Intern. Jour. of Algebra and Computation 4 (1994), no. 1-2, 1-308. MR 95h:20051

[Iv3] S.V. Ivanov, On some finiteness conditions in group and semigroup theory, Semigroup Forum 48 (1994), no. 1, 28-37. MR 94k:20102

[KN] Kourovka Notebook: Unsolved problems in group theory, 12th Ed., Novosibirsk, 1992. MR 95d:20001

[Ks] A.I. Kostrikin, Around Burnside, Nauka, Moscow, 1986. MR 89d:20032

[LS] R.C. Lyndon and P.E. Schupp, Combinatorial group theory, Springer-Verlag, 1977. MR $\mathbf{5 8 : 2 8 1 8 2}$

[Ly] I.G. Lysënok, On some algorithmic properties of hyperbolic groups, Math. USSR Izvestiya 35 (1990), no. 1, 145-163. MR 91b:20041

[MKS] W. Magnus, J. Karras, and D. Solitar, Combinatorial group theory, Interscience Pub., John Wiley and Sons, 1966. MR 34:7617

[NA] P.S. Novikov and S.I. Adian, On infinite periodic groups, I, II, III, Math. USSR Izvestiya 32 (1968), 212-244, 251-524, 709-731. MR 39:1532

[Ol1] A.Yu. Ol'shanskii, On the Novikov-Adian theorem, Math. USSR Sbornik 118 (1982), no. 2, 203-235. MR 83m:20058

[Ol2] A.Yu. Ol'shanskii, Geometry of Defining Relations in Groups, Nauka, Moscow, 1989; English translation in Math. and Its Applications (Soviet series) 70 (Kluwer Acad. Publishers, 1991). MR 93g:20071

[O13] A.Yu. Ol'shanskii, Embedding of countable periodic groups into 2-generator simple periodic groups, Ukranian Math. Jour. 43 (1991), no. 7-8, 980-986. MR 93d:20074

[O14] A.Yu. Ol'shanskii, Almost every group is hyperbolic, Intern. Jour. of Algebra and Computation 2 (1992), no. 1, 1-17. MR 93j:20068

[Ol5] A.Yu. Ol'shanskii, Periodic quotient groups of hyperbolic groups, Math. USSR Sbornik 72 (1992), no. 2, 519-541.

[Ol6] A. Yu. Ol'shanskii, On residualing homomorphisms and G-subgroups of hyperbolic groups, Intern. Jour. of Algebra and Computation 3 (1993), no. 4, 365-409. MR 94i:20069

[Rt] J.J. Rotman, An introduction to the theory of groups, 4th ed., Springer-Verlag, 1995. MR 95:05

[Sl] A. Selberg, On discontinuous groups in higher-dimensional symmetric spaces, Colloq. Function Theory, Bombay, 1960, pp. 147-164. MR 24:A188

[Zl1] E.I. Zelmanov, Solution of the restricted Burnside problem for groups of odd exponent, Math. USSR Izvestiya 36 (1991), no. 1, 41-60. MR 91i:20037

[Z12] E.I. Zelmanov, A solution of the restricted Burnside problem for 2-groups, Math. USSR Sbornik 72 (1992), no. 2, 543-565.

Department of Mathematics, University of Illinois at Urbana-Champaign, 1409 West Green Street, Urbana, Illinois 61801

E-mail address: ivanov@math.uiuc.edu

Department of Mechanics and Mathematics, Moscow State University, Moscow 119899, Russia

E-mail address: olsh@nw.math.msu.su 